Mathematical Medicine and Biology Page 1 of 55

doi:10.1093/imammb/dqnxxx

\title{
Predicting tyrosinaemia: a mathematical model of 4-hydroxyphenylpyruvate dioxygenase inhibition by nitisinone in rats
}

\author{
JOHN P. WARD* \\ Department of Mathematical Sciences, Loughborough University, LE11 3TU, United \\ Kingdom \\ ${ }^{*}$ Corresponding author: john.ward@lboro.ac.uk \\ JOANNE L. DUNSTER \\ Department of Mathematics \& Statistics, University of Reading, PO Box 220, Reading, RG6 \\ 6AX, United Kingdom \\ GIANNE DERKS \\ Department of Mathematics, University of Surrey, Guildford, GU2 7XH, United Kingdom \\ Pratibha Mistry and José D. Salazar \\ Product Safety, Syngenta, Jealott's Hill International Research Centre, Bracknell, RG42 6EY, \\ United Kingdom.
}

\begin{abstract}
Nitisinone or 2-(2-nitro-4-trifluoromethylbenzoyl)cyclohexane-1,3-dione, is a reversible inhibitor of 4hydroxyphenylpyruvate dioxygenase (HPPD), an enzyme important in tyrosine catabolism. Today, nitisinone is successfully used to treat Hereditary Tyrosinaemia type 1, although its original expected role was as a herbicide. In laboratory animals, treatment with nitisinone leads to the elevation of plasma tyrosine (tyrosinaemia). In rats and Beagle dogs, repeat low-dose exposure to nitisinone leads to corneal opacities whilst similar studies in the mouse and Rhesus monkey showed no comparable toxicities or other treatment related findings. The differences in toxicological sensitivities have been related to the upper limit of the concentration of tyrosine that accumulates in plasma, which is driven by the amount/activity of tyrosine aminotransferase.

A physiologically based, pharmacodynamics ordinary differential equation model of HPPD inhibition to bolus exposure of nitisinone in vivo is presented. Going beyond traditional approaches, asymptotic analysis is used to separate the different timescales of events involved in HPPD inhibition and tyrosinaemia. This analysis elucidates, in terms of the model parameters, a critical inhibitor concentration (at which tyrosine concentration starts to rise) and highlights the contribution of in vitro measured parameters to events in an in vivo system. Furthermore, using parameter-fitting methods, a systematically derived reduced model is shown to fit well to rat data, making explicit how the parameters are informed by such data. This model in combination with in vitro descriptors has potential as a surrogate for animal experimentation to predict tyrosinaemia, and further development can extend its application to other related medical scenarios.
\end{abstract}

Keywords: nitisinone, 4-hydroxyphenylpyruvate dioxygenase, tyrosinaemia, rat, mathematical model, $\mathrm{PBPK} / \mathrm{PD}$, asymptotic analysis.

\section{Introduction}

Nitisinone or 2-(2-nitro-4-trifluoromethylbenzoyl)cyclohexane-1,3-dione was originally discovered as a member of the triketone class of herbicides (Lock et al., 1998). These herbicides are potent inhibitors of 


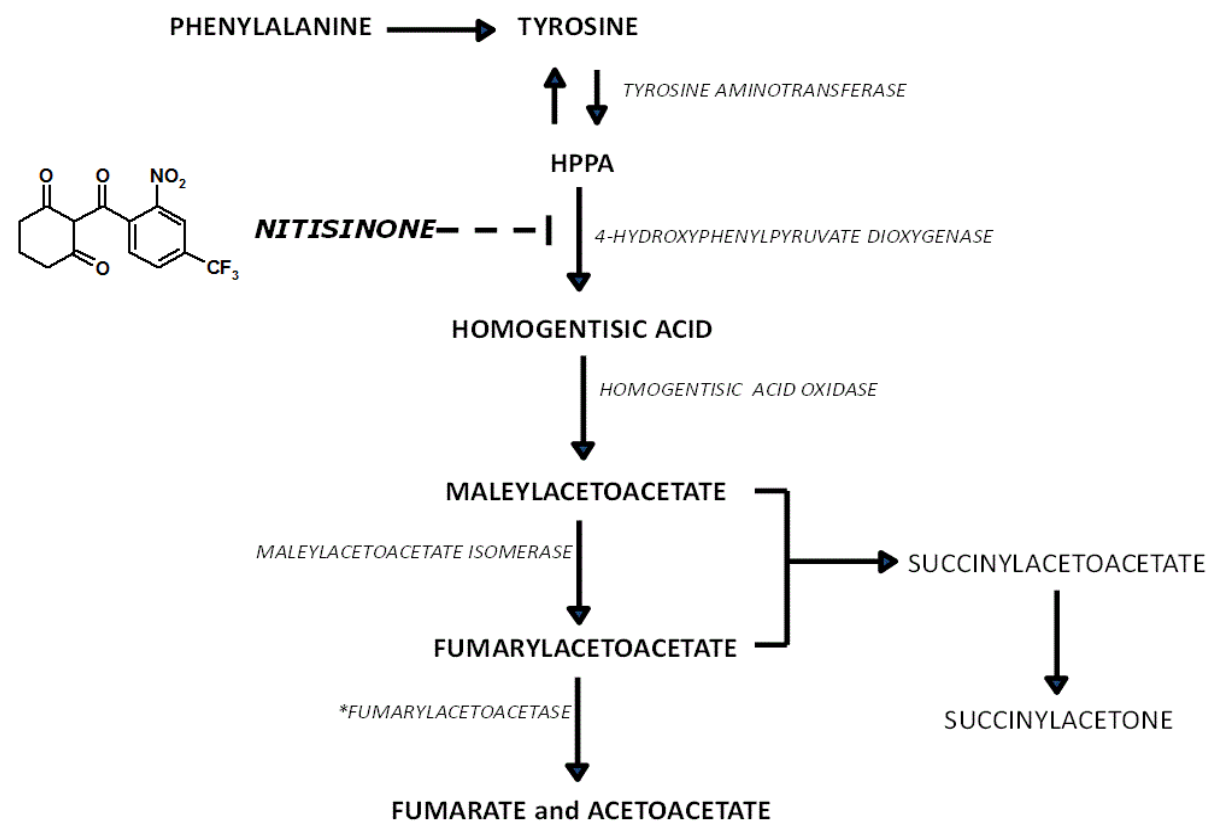

FIG. 1. Tyrosine catabolism pathway indicating fumarylacetatoacetase activity $(*)$, the enzyme deficient in type I tyrosinaemia patients. The schematic also indicates the inhibition of 4-hydroxyphenylpyruvate dioxygenase (HPPD) by nitisinone, thus preventing the generation of maleyacetoacetate and fumarylacetoacetate and their metabolites.

plant 4-hydroxyphenylpyruvate dioxygenase (HPPD), an enzyme important in the synthesis of plastoquinones and $\alpha$-tocopherol (Prisbylla et al., 1993; Schulz et al., 1993; Ellis et al., 1995). In mammals, HPPD is mainly present in the liver and is involved in the catabolism of tyrosine, an amino acid found in proteins from the diet. Today, nitisinone is used in the clinic to treat a rare hereditary metabolic disorder (Hereditary Tyrosinaemia type 1), where patients present with symptoms caused by a deficiency of the enzyme fumarylacetoacetase, an enzyme in tyrosine catabolism (Figure 1) (Lock et al., 1992; Holme \& Lindstedt S., 1998). Such a deficiency leads to the accumulation of fumarylacetoacetate, maleyacetoacetoacetate and their associated derivatives. These electrophilic metabolites contribute to progressive liver and kidney dysfunction as well as porphyria in patients. Treatment of patients with nitisinone prevents the formation of these harmful metabolites (such as succinylacetone) by blocking an earlier step in the tyrosine catabolism pathway. This form of treatment coupled with a tyrosine and phenylalaninerestricted diet provides a favourable outcome, particularly in patients when diagnosed early (McKiernan, 2006); liver failure is controlled in $90 \%$ of cases and other disease-related manifestations are well managed. Overall, nitisinone is well-tolerated in patients and has changed the clinical management of Heredatary Tyrosinaemia type I by dramatically reducing the need for liver transplantation.

In laboratory animals, treatment with HPPD inhibitors leads to the inhibition of tyrosine catabolism and consequently the elevation plasma tyrosine (tyrosinaemia). In rats and Beagle dogs, the main toxi- 
cological endpoints observed following short-term repeat dose exposure with nitisinone include corneal opacities at low doses. Similar studies in the mouse and Rhesus monkey showed no comparable toxicities or other treatment related findings (Lock et al., 1998). Interestingly, the differences in toxicological sensitivities between these species cannot be readily explained by molecular structural or sequence differences in the HPPD enzyme as these features are highly conserved (Lock et al., 2006). However, the upper limit of the concentration of tyrosine that accumulates in plasma does vary between mammalian species, and studies have described these differences to be driven by the amount/activity of tyrosine aminotransferase (TAT, see Figure 1), an enzyme important in the conversion of tyrosine to hydroxyphenyl pyruvic acid (HPPA) (Lock et al., 1996, 1998, 2000; Lewis R.W. \& Botham J.W., 2013). Interestingly, humans deficient in TAT, referred to as Richner-Hanhart syndrome, also present with corneal opacities if dietary phenylalanine and tyrosine is not restricted (Goldsmith, 1983; Sammartino et al., 1987). In the clinic, plasma tyrosine concentrations in patients with type I tyrosinaemia are maintained at less than 500 mole/L (McKiernan, 2006; Mitchell et al., 2001). Since we are primarily interested in understanding the intrinsic dynamics of the tyrosine catabolism pathway, we will assume that the concentrations of the TAT enzyme will remain constant in the chosen animal system during HPPD inhibition. However external influences on TAT (e.g., circadian rhythms, diet, etc.) can be readily included and will be discussed in the last Section.

Animal studies are often used to understand the potency of new HPPD inhibitors via the extent of exposure in circulating blood and the resulting tyrosinaemia. In addition, standard physiologically-based pharmacokinetic-pharmacodynamic (PBPK-PD) approaches can offer information on the kinetics of the inhibitor concentration and tyrosine dynamics across organs which are represented as compartments. In this paper we extend the classical PBPK-PD approach by modelling the most relevant enzymatic reactions in the liver in greater detail than is usually done (see Section 2) and utilizing asymptotic analysis to determine the critical events that take place at each of the time scales of the full inhibition-recovery process (see Section 3). This kind of analysis allows us to understand qualitatively the phenomena under study without precise measurements on the parameter values. In Section 2, by making some generic assumptions based on nitisinone experimental data, we are able to derive a number of interesting consequences from the model. The asymptotic analysis carried out in Section 3 then provides key insights such as a prediction of the critical inhibitor concentration at which tyrosine concentration starts to rise, Equation (3.35), or a simple description of the inhibitor profile, Equation (4.1). Then in Section 4 we fit the model to nitisinone experimental data, in order to estimate actual parameter values. The results indicate that only certain parameter combinations are identifiable from the available experimental data. Finally, in Section 5, we explain how to use our model as a virtual test bed for the development of new HPPD inhibitors by utilizing information already available together with in vitro measurements. As such, the key utility of this work is to serve as a surrogate for animal experimentation for new HPPD inhibitors in early research, as well as a base model to adapt for repeat dose exposure scenarios and to support human risk assessment activities.

\section{Model Formulation}

In this section we construct a kinetic model of the tyrosine degradation pathway and the effects of a HPPD inhibitor. The model consists of a system of ordinary differential equations which describe the changes in concentration of the various molecules involved in the reactions. A schematic of these reactions is shown in Figure 2 and the variables and parameters are listed in Tables 1 and 2 respectively. The appendix lists some parameter values from the literature.

The model is subdivided into three compartments representing blood (volume $V_{B}$ ), tissue (volume 
4 of 55

\begin{tabular}{|c|c|c|}
\hline Variable & Description & Unit \\
\hline$T_{B}$ & Tyrosine in blood & $\mathrm{M}$ \\
$T_{L}$ & Tyrosine in liver & $\mathrm{M}$ \\
$T_{T}$ & Tyrosine in tissue & $\mathrm{M}$ \\
$H_{p}$ & Hydroxyphenyl pyruvic acid (HPPA) & $\mathrm{M}$ \\
$H_{o}$ & 4-Hydroxyphenylpyruvate acid (HPPD) & $\mathrm{M}$ \\
$I_{B}$ & HPPD inhibitor in blood & $\mathrm{M}$ \\
$I_{L}$ & HPPD inhibitor in liver & $\mathrm{M}$ \\
$I_{T}$ & HPPD inhibitor in tissue & $\mathrm{M}$ \\
$A$ & Tyrosine aminotransferase (TAT) & $\mathrm{M}$ \\
$R$ & Homogentistic acid & $\mathrm{M}$ \\
$C$ & Complex formed from $H_{o}$ and $H_{p}$ & $\mathrm{M}$ \\
\hline
\end{tabular}

Table 1. Definitions and units of the variables. In the units column, $\mathrm{M}$ stands for molar (moles per liter.

$V_{T}$ ) and the liver (volume $V_{L}$ ).

- Tyrosine, an amino acid that forms part of all proteins and is a precursor of neurotransmitters, is produced by muscle tissue (rate $T_{1}$ ) and obtained from diet (rate $T_{0}$ ). It is present in blood (concentration $\left.T_{B}\right)$ and tissue $\left(T_{T}\right)$, but mainly in the liver $\left(T_{L}\right)$ where it is broken down, (note that this implies that the total measure of tyrosine in the blood is given by $V_{B} T_{B}$ and similar measures apply for tyrosine in the tissue and liver). We assume tyrosine decays in the tissue, blood and liver at rates $\delta_{T_{T}}, \delta_{T_{B}}, \delta_{T_{L}}$ respectively. In our model tyrosine can be transferred between the tissue and blood compartments (rate $\alpha_{4}$, partition coefficient $\mu_{4}$ ) and between the blood and the liver (rate $\alpha_{1}$, partition coefficient $\mu_{1}$ ).

- Once within the liver, tyrosine conversion to hydroxyphenyl pyruvic acid (HPPA, $H_{p}$ ) is thought to be controlled by the enzyme tyrosine aminotransferase (TAT, $A$ ) at rate $k_{-1}$; and is reversible at rate $k_{1}$ that is independent of TAT. The turnover of the enzyme TAT is assumed to be independent of the other components of the model, being produced, for simplicity, at a constant rate $\left(T_{A}\right)$. HPPA and TAT are assumed to decay naturally at rates $\delta_{p}$ and $\delta_{A}$ respectively.

- 4-hydroxyphenylpyruvate dioxygenase (HPPD, $H_{0}$ ) is produced in the liver (at a background rate of $B_{0}$ ) where it catalyzes, but is not depleted by, the conversion of HPPA (rate $\delta_{H}$ ) to the downstream by-product homogentistic acid $(R)$. HPPD and Homogenestic Acid decay at rates $\delta_{o}$ and $\delta_{S}$ respectively.

- The HPPD inhibitor is administered directly into the blood $\left(I_{B}\right.$, for the purpose of this paper we will not consider explicitly inhibitor plasma binding) and can transfer (at rate $\alpha_{3}$, partition coefficient $\mu_{3}$ ) to the tissue $\left(I_{T}\right)$ and (at rate $\alpha_{2}$, partition coefficient $\mu_{2}$ ) to the liver $\left(I_{L}\right)$. Once within the liver the HPPD inhibitor forms a complex $(C)$ with HPPD preventing it metabolyzing HPPA to homogentistic acid. The complex is formed at rate $k_{2}$ (that is reversible (rate $k_{-2}$ )) and is assumed to decay at rate $\delta_{C}$.

Thus the equations representing the eleven variables are: 


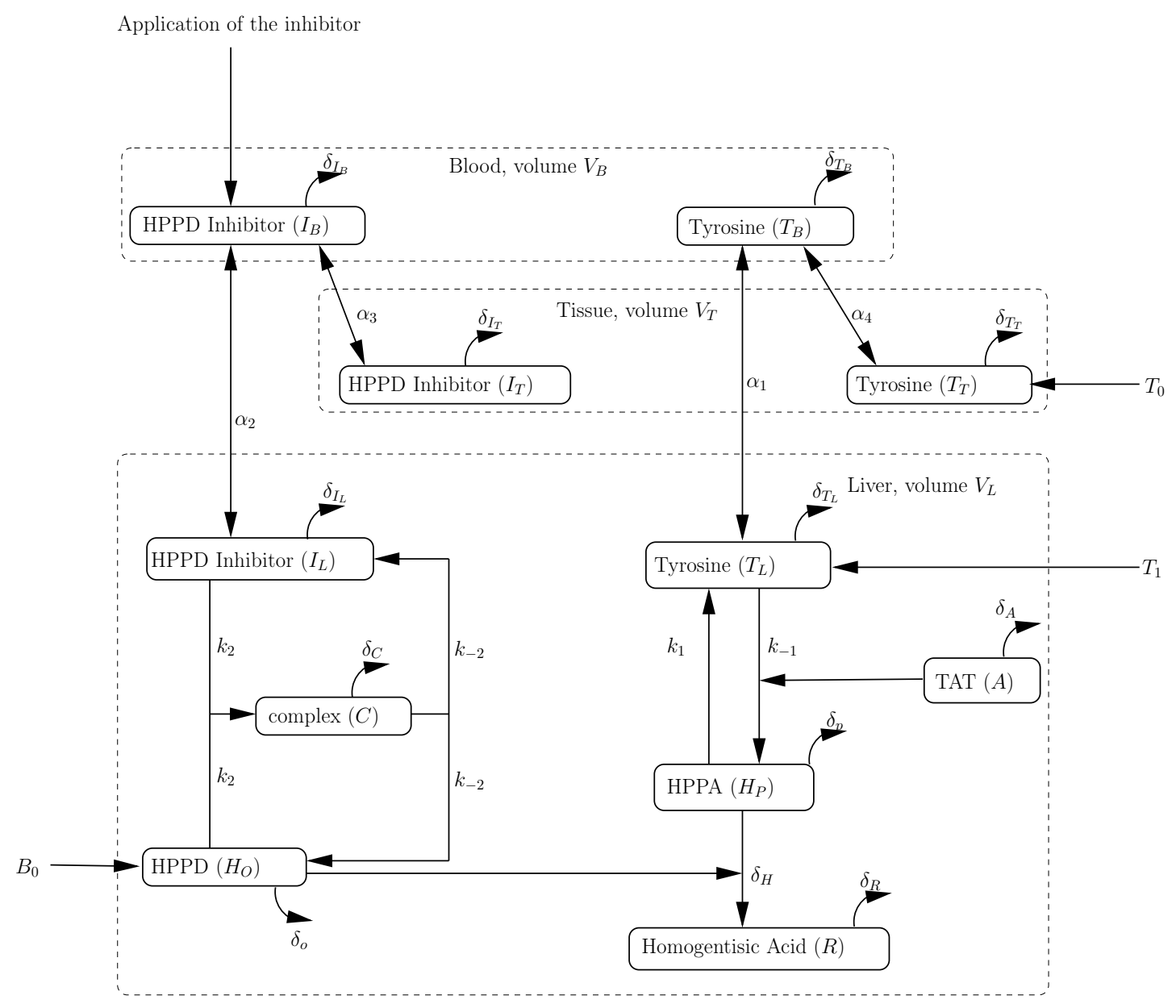

FIG. 2. A schematic depicting the degradation pathway of tyrosine and its inhibition via a HPPD inhibitor. Once tyrosine is within the liver it is aided in its break down to HPPA by the enzyme TAT. HPPD then catalyzes its further breakdown to homogentistic acid. HPPD inhibitors form a complex with HPPD within the liver inhibiting HPPD's ability to catalyze the breakdown of tyrosine into its downstream products.

$$
\begin{aligned}
V_{T} \frac{d T_{T}}{d t} & =T_{0}-\alpha_{4}\left(T_{T}-\mu_{4} T_{B}\right)-V_{T} \delta_{T_{T}} T_{T} \\
V_{B} \frac{d T_{B}}{d t} & =\alpha_{1}\left(T_{L}-\mu_{1} T_{B}\right)+\alpha_{4}\left(T_{T}-\mu_{4} T_{B}\right)-V_{B} \delta_{T_{B}} T_{B}, \\
V_{L} \frac{d T_{L}}{d t} & =T_{1}-\alpha_{1}\left(T_{L}-\mu_{1} T_{B}\right)+V_{L} k_{1} H_{P}-V_{L} k_{-1} A T_{L}-V_{L} \delta_{T_{L}} T_{L}, \\
V_{L} \frac{d H_{P}}{d t} & =-V_{L} k_{1} H_{P}+V_{L} k_{-1} A T_{L}-V_{L} \delta_{P} H_{P}-V_{L} \delta_{H} H_{O} H_{P}, \\
V_{L} \frac{d H_{O}}{d t} & =B_{0}-V_{L} k_{2} H_{O} I_{L}+V_{L} k_{-2} C-V_{L} \delta_{O} H_{O},
\end{aligned}
$$


6 of 55

$$
\begin{aligned}
V_{L} \frac{d R}{d t} & =V_{L} \delta_{H} H_{O} H_{P}-V_{L} \delta_{R} R \\
V_{L} \frac{d A}{d t} & =A_{0}-V_{L} \delta_{A} A \\
V_{L} \frac{d C}{d t} & =V_{L} k_{2} H_{O} I_{L}-V_{L} k_{-2} C-V_{L} \delta_{C} C \\
V_{T} \frac{d I_{T}}{d t} & =\alpha_{3}\left(\mu_{3} I_{B}-I_{T}\right)-V_{T} \delta_{I_{T}} I_{T} . \\
V_{B} \frac{d I_{B}}{d t} & =\alpha_{2}\left(I_{L}-\mu_{2} I_{B}\right)-\alpha_{3}\left(\mu_{3} I_{B}-I_{T}\right)-V_{B} \delta_{I_{B}} I_{B}, \\
V_{L} \frac{d I_{L}}{d t} & =-\alpha_{2}\left(I_{L}-\mu_{2} I_{B}\right)-V_{L} k_{2} H_{O} I_{L}+V_{L} k_{-2} C-V_{L} \delta_{I_{L}} I_{L},
\end{aligned}
$$

To close this system a set of initial conditions are needed. We assume that the inhibitor is administered as a single bolus dose, concentration $I_{0}$, into the blood at $t=0$, so that the non-inhibitor variables will be at homeostatic levels. We note that $I_{0}$ is the initial value of available inhibitor, based on the assumption that inhibitor binding with plasma is rapid in comparison to transfer to the liver and tissue. Due to feeding pattern influencing the source term $T_{1}$ the initial conditions are to some extent arbitrary. However, in the simulations and analysis to follow we assume, as a first approximation, that $T_{1}$ is constant (e.g. its mean concentration), and that initially the non-inhibitor related variables are in a state of equilibrium. In the absence of the inhibitor, the steady-state for HPPD can be independently determined $\left(H_{O}=B_{0} / V_{L} \delta_{O}\right)$, which means that equations (2.1a)-(2.1d) form a linear system for the steady-state concentrations of tyrosine and HPPA; due to their somewhat unwieldy form we denote them here using a superscript $S S$ and omit details of $T_{B}^{S S}$ and $H_{P}^{S S}$ for brevity; leading order dimensionless expressions for $T_{B}^{S S}$ and $H_{P}^{S S}$ for the biologically relevant case of $V_{B} / V_{T} \ll 1$ are presented in Section 3. The initial conditions are thus

$$
\begin{gathered}
T_{T}=\frac{\alpha_{4} \mu_{4} T_{B}^{S S}+T_{0}}{V_{T} \delta_{T_{T}}+\alpha_{4}}, \quad T_{B}=T_{B}^{S S}, \quad T_{L}=\frac{H_{P}^{S S}\left(B_{0} \delta_{H}+V_{L} \delta_{O}\left(\delta_{P}+k_{1}\right)\right)}{V_{L} A^{S S} \delta_{O} k_{-1}}, \quad H_{P}=H_{P}^{S S}, \\
H_{O}=H_{O}^{S S}=\frac{B_{0}}{V_{L} \delta_{O}}, \quad R=\frac{\delta_{H} B_{0} H_{P}^{S S}}{\delta_{O} \delta_{R}}, \quad A=A^{S S}=\frac{A_{0}}{\delta_{A} V_{L}}, \\
C=0, \quad I_{L}=0, \quad I_{B}=I_{0}, \quad I_{T}=0,
\end{gathered}
$$

at $t=0$. We note that from (2.1g) these initial conditions imply $A \equiv A^{S S}$ for all time and we henceforth omit the differential equation of $A$ (which corresponds to TAT concentration) in future discussions.

\subsection{Parameter sizes and non-dimensionalisation}

Table 2 lists all of the parameters in the proposed model. Though estimates for certain parameters are readily available (e.g. liver, blood and tissue volumes for various animals, see Brown et al. (1997)), others can not be obtained directly from the current experimental literature. The time-course data for a single oral dose scenario in the rat shown in Figure 7 indicates that tyrosine concentrations increase 2030 fold over a 24 hour period, returning to homeostatic concentrations after about 3 days. Though these data reveal the approximate timescales of events, it is not clear how this informs the model parameters. Amongst the outcomes of the timescale analysis in Section 3 is to establish precisely which parameters 


\begin{tabular}{|c|c|c|}
\hline Parameter & Description & Unit \\
\hline$\alpha_{1}$ & Tyrosine mass exchange rate between liver and blood & $\mathrm{LS}^{-1}$ \\
\hline$\alpha_{2}$ & Inhibitor mass exchange rate conversion between liver and blood & $\mathrm{LS}^{-1}$ \\
\hline$\alpha_{3}$ & Inhibitor mass exchange rate conversion between tissue and blood & $\mathrm{LS}^{-1}$ \\
\hline$\alpha_{4}$ & Tyrosine mass exchange rate conversion between tissue and blood & $\mathrm{LS}^{-1}$ \\
\hline$\mu_{1}$ & Tyrosine partition coefficient between blood and liver & none \\
\hline$\mu_{2}$ & Inhibitor partition coefficient between blood and liver & none \\
\hline$\mu_{3}$ & Inhibitor partition coefficient between blood and tissue & none \\
\hline$\mu_{4}$ & Tyrosine partition coefficient between blood and tissue & none \\
\hline$\delta_{C}$ & Decay rate constant of HPPD/inhibitor complex & $\mathrm{S}^{-1}$ \\
\hline$\delta_{I_{B}}$ & Decay rate constant of inhibitor in blood & $S^{-1}$ \\
\hline$\delta_{I_{L}}$ & Decay rate constant of inhibitor in liver & $\mathrm{S}^{-1}$ \\
\hline$\delta_{I_{T}}$ & Decay rate constant of inhibitor in tissue & $\mathrm{S}^{-1}$ \\
\hline$\delta_{A}$ & Decay rate constant of TAT & $S^{-1}$ \\
\hline$\delta_{O}$ & Decay rate constant of HPPD & $\mathrm{S}^{-1}$ \\
\hline$\delta_{P}$ & Decay rate constant of HPPA & $\mathrm{S}^{-1}$ \\
\hline$\delta_{H}$ & Breakdown of HPPA to HG acid & $\mathrm{M}^{-1} \mathrm{~S}^{-1}$ \\
\hline$\delta_{R}$ & Decay rate constant of homogentistic acid & $\mathrm{S}^{-1}$ \\
\hline$\delta_{T_{B}}$ & Decay rate constant of tyrosine in blood & $S^{-1}$ \\
\hline$\delta_{T_{L}}$ & Decay rate constant of tyrosine in liver & $S^{-1}$ \\
\hline$\delta_{T_{T}}$ & Decay rate constant of tyrosine in tissue & $\mathrm{S}^{-1}$ \\
\hline$A_{0}$ & Maximal rate of TAT production & $\mathrm{MLS}^{-1}$ \\
\hline$B_{0}$ & Background production of HPPD & $\mathrm{MLS}^{-1}$ \\
\hline$T_{0}$ & Production of tyrosine in tissue & $\mathrm{MLS}^{-1}$ \\
\hline$T_{1}$ & Tyrosine intake rate in liver from diet & $\mathrm{MLS}^{-1}$ \\
\hline$V_{B}$ & Volume of blood & $\mathrm{L}$ \\
\hline$V_{L}$ & Volume of liver & $\mathrm{L}$ \\
\hline$V_{T}$ & Volume of tissue & $\mathrm{L}$ \\
\hline$k_{1}$ & Formation of tyrosine from HPPA & $\mathrm{S}^{-1}$ \\
\hline$k_{-1}$ & Breakdown of tyrosine to HPPA & $\mathrm{M}^{-1} \mathrm{~S}^{-1}$ \\
\hline$k_{2}$ & "on rate" for HPPD/inhibitor binding & $\mathrm{MS}^{-1}$ \\
\hline$k_{-2}$ & “off rate” for HPPD/inhibitor & $\mathrm{S}^{-1}$ \\
\hline$I_{0}$ & Initial bolus inhibitor dose, $I_{B}(0)$ & $\mathrm{M}$ \\
\hline
\end{tabular}

Table 2. Parameters, definitions and units. In the units column, $\mathrm{M}$ stands for molar, $\mathrm{L}$ for liters and $\mathrm{S}$ for seconds. 
8 of 55

can be reliably estimated using this data for rats. In order to do this analysis we non-dimensionalise the system of equations (2.1a)- $(2.1 \mathrm{~m})$ in such a way that the magnitude of new dimensionless parameters are all expressed in terms of a small parameter, $\varepsilon$, defined as

$$
\varepsilon=\frac{V_{B}}{V_{T}}
$$

which is the volume ratio of the blood and tissue compartments; for the rat $\varepsilon=V_{B} / V_{T} \approx 0.04$, and $V_{B} / V_{L} \approx 2=O(1)$ (see Brown et al. (1997)). As many of the parameters are unknown, we will instead make informed estimates on the order of magnitude of the dimensionless versions. These estimates are broadly based on the fact that components of the model clearly operate on different timescales; i.e., we expect that the reactions are generally fast (equilibration over $O$ (seconds-minutes)), circulation and compartmental exchange of tyrosine and inhibitor are not so fast $(O(10$ minutes-hours $))$ and clearance and recovery rates will be relatively slow ( $O(10$ hours-days), as suggested by the time-course data. Further to this, the magnitude of parameters are chosen so that features in the data for the rat can be reliably reproduced, namely that (1) blood tyrosine increases by an order of magnitude (a factor of $O(1 / \varepsilon)$ ) due to the inhibitor, (2) the maximum tyrosine concentration is independent of inhibitor dose (within the range used in the experiments) and (3) the sources of tyrosine from the diet and from production in tissue are at about the same concentration. It turns out that imposing these constraints narrows down significantly the possible magnitudes of the dimensionless parameters in the model.

The experimental time-course data available (Figure 7) suggests that the inhibitor and its effects occur over a number of hours and we rescale time to this long-time scale, using

$$
t=\frac{\hat{t}}{\delta_{I_{B}}}
$$

where $\hat{t}$ is the dimensionless time variable. From the inhibitor clearance rate, estimated to be about $1.5 \mathrm{day}^{-1}$ (see Figure 7), then $\hat{t}=1$ represents about 16 hours. At homeostasis, the tyrosine concentration is expected to be of the same order of magnitude throughout the body (unfortunately measurements are only available for the aqueous humor of the rat, see Lock et al. (1996)). We scale tyrosine, HPPD and homogentistic acid with the scaling parameter $T^{*}=T_{0} / \delta_{T_{T}} V_{T}$, which is the steady tissue tyrosine concentration in the absence of exchange in the blood. We scale HPPD and HPPD-drug complex with the homeostatic HPPD concentration, namely $H_{O}^{S S}=B_{0} / \delta_{0} V_{L}$. In single dose oral exposure experiments (see Figure 7), assuming rapid absorption, a typical quantity of inhibitor is about $1 \mathrm{mg} / \mathrm{kg}$, thereby for a $0.3 \mathrm{~kg}$ rat, this represents an initial blood concentration of $I_{B}(0)=I_{0}^{\ddagger} \approx 0.3 /\left(M_{I} V_{B}\right) \mathrm{M}$, where $M_{I}$ is the mass per mole of inhibitor; this is in fact an upper bound of available inhibitor $I_{0}$ because, as noted earlier, we are not accounting for inhibitor plasma binding. Though $I_{0}$ or $I_{0}^{\ddagger}$ are natural scaling parameters for inhibitor, we will instead scale $I_{i}$ with $H_{O}^{S S}$ so that the dimensionless quantity $I_{i} / H_{O}$ is the molar ratio between available inhibitor and HPPD. The system is thus scaled using

$$
T_{i}=T^{*} \widehat{T}_{i}, \quad H_{P}=T^{*} \widehat{H}_{P}, \quad R=T^{*} \widehat{R}, \quad H_{O}=H_{O}^{S S} \widehat{H}_{O}, \quad C=H_{O}^{S S} \widehat{C}, \quad I_{i}=H_{O}^{S S} \widehat{I}_{i},
$$

where the hatted quantities are the dimensionless variables and $i \in\{T, B, L\}$.

We can infer from the experimental time-course data (again see Figure 7) that recovery from inhibitor intake takes about 2-3 days, which suggests that the inhibitor metabolism/decay and the turnover of HPPD and tyrosine operate on an $\hat{t}=O(1)$ timescale. Blood circulation occurs on a timescale of 
$O$ (minutes) and we assume that the blood-organ mass exchange parameters are of $O(1 / \varepsilon)$ in size. Relative to these processes, chemical reactions are expected to occur much faster. Due to the assumed rapidity of the two way tyrosine-HPPA reaction, tyrosine intake rate from the diet must be $O\left(1 / \varepsilon^{2}\right)$ in order for liver tyrosine concentration to be the same order as the blood and tissue. We assume that the tyrosine-HPPA reaction equilibrates in a timescale of $O\left(\varepsilon^{2}\right)$ (in a about a minute) and likewise assume HPPA conversion to homogentisic acid occurs at about the same rate; hence the corresponding parameters are assumed to be $O\left(1 / \varepsilon^{2}\right)$. Due to the high affinities between the inhibitor and HPPD we assume this is the most rapid reaction, occurring at an $O\left(1 / \varepsilon^{3}\right)$ rate (i.e. reaction saturates in a timescale of about a second); we note that the inhibitor-HPPD complex is relatively stable and the reverse reaction is much slower. The greater volume of tissue means that it acts as a significant sink to the inhibitor; in order to get sufficient inhibitor into the liver, so that the maximum tyrosine is insensitive to the dose, we require the initial blood concentration to be $O(1 / \varepsilon)$, this means on equilibration the inhibitor concentration is $O(1)$ in the liver as required for it to be most effective. On this basis we define the dimensionless parameters as follows,

$$
\begin{aligned}
& \frac{1}{\varepsilon^{2}}\left\{\hat{k}_{1}, \hat{k}_{-1}, \hat{\delta}_{H}, \widehat{\delta}_{R}\right\}=\frac{1}{\delta_{I_{B}}}\left\{k_{1}, k_{-1} A^{S S}, \delta_{H} H_{O}^{S S}, \delta_{R}\right\}, \\
& \frac{1}{\varepsilon}\left\{\hat{\alpha}_{1}, \hat{\alpha}_{2}, \hat{\alpha}_{3}, \hat{\alpha}_{4}\right\}=\frac{1}{V_{T} \delta_{I_{B}}}\left\{\alpha_{1}, \alpha_{2}, \alpha_{3}, \alpha_{4}\right\} \\
&\left\{\widehat{\delta}_{T_{T}}, \widehat{\delta}_{T_{B}}, \widehat{\delta}_{T_{L}}, \widehat{\delta}_{P}, \widehat{\delta}_{I_{T}}, \widehat{\delta}_{I_{L}}, \hat{\delta}_{O}, \hat{k}_{-2}\right\}=\frac{1}{\delta_{I_{B}}}\left\{\delta_{T_{T}}, \delta_{T_{B}}, \delta_{T_{L}}, \delta_{P}, \delta_{I_{T}}, \delta_{I_{L}}, \delta_{O}, k_{-2}\right\} \\
& \frac{\hat{k}_{2}}{\varepsilon^{3}}=\frac{k_{2} H_{O}^{S S}}{\delta_{I_{B}}}, \quad \frac{S_{1}}{\varepsilon^{2}}=\frac{T_{1}}{\delta_{I_{B}} V_{L} T^{*}}, \quad v=\frac{V_{B}}{V_{L}}, \quad \theta=\frac{I_{0}}{H_{O}^{S S}} .
\end{aligned}
$$

We note that the only dimensionless parameter that has an explicit dependency on the concentration of $\mathrm{TAT}\left(A^{S S}\right)$ is $\hat{k}_{-1}$; increasing this parameter is the equivalent to raising TAT. It is thought that the HPPDinhibitor complex is stable so that the breakdown to its components is expected to occur very slowly; the choice of scaling $k_{-2}$ is such that it is the largest order of magnitude to be visible in the analysis of Section 3, but has no meaningful effects on the dynamics (i.e. setting $\widehat{k}_{-2}=0$ will make no difference to the results).

Applying all these scalings on the variables and parameters leads to the system of dimensionless equations (the hats have been dropped from now on)

$$
\begin{aligned}
\frac{d T_{T}}{d t} & =\delta_{T_{T}}\left(1-T_{T}\right)+\alpha_{4}\left(\mu_{4} T_{B}-T_{T}\right), \\
\varepsilon \frac{d T_{B}}{d t} & =\alpha_{1}\left(T_{L}-\mu_{1} T_{B}\right)+\alpha_{4}\left(T_{T}-\mu_{4} T_{B}\right)-\varepsilon \delta_{T_{B}} T_{B}, \\
\varepsilon^{2} \frac{d T_{L}}{d t} & =S_{1}-\varepsilon \alpha_{1} v\left(T_{L}-\mu_{1} T_{B}\right)+k_{1} H_{P}-k_{-1} T_{L}-\varepsilon^{2} \delta_{T_{L}} T_{L}, \\
\varepsilon^{2} \frac{d H_{P}}{d t} & =-k_{1} H_{P}+k_{-1} T_{L}-\delta_{H} H_{O} H_{P}-\varepsilon^{2} \delta_{P} H_{P}, \\
\varepsilon^{3} \frac{d H_{O}}{d t} & =\varepsilon^{3} \delta_{O}\left(1-H_{O}\right)-k_{2} H_{O} I_{L}+\varepsilon^{3} k_{-2} C, \\
\varepsilon^{2} \frac{d R}{d t} & =\delta_{H} H_{O} H_{P}-\delta_{R} R,
\end{aligned}
$$


10 of 55

$$
\begin{aligned}
\varepsilon^{3} \frac{d C}{d t} & =k_{2} H_{O} I_{L}-\varepsilon^{3} k_{-2} C-\varepsilon^{3} \delta_{C} C, \\
\frac{d I_{T}}{d t} & =-\alpha_{3}\left(I_{T}-\mu_{3} I_{B}\right)-\delta_{I_{T}} I_{T}, \\
\varepsilon \frac{d I_{B}}{d t} & =\alpha_{2}\left(I_{L}-\mu_{2} I_{B}\right)+\alpha_{3}\left(I_{T}-\mu_{3} I_{B}\right)-\varepsilon I_{B}, \\
\varepsilon^{3} \frac{d I_{L}}{d t} & =-\varepsilon^{2} \alpha_{2} v\left(I_{L}-\mu_{2} I_{B}\right)-k_{2} H_{O} I_{L}+\varepsilon^{3} k_{-2} C-\varepsilon^{3} \delta_{I_{L}} I_{L} .
\end{aligned}
$$

\subsection{Initial conditions}

The dimensionless form of the initial conditions (2.1) are

$$
\begin{gathered}
T_{T}=\frac{\alpha_{4} \mu_{4} T_{B}^{S S}+\varepsilon \delta_{T_{T}}}{\alpha_{4}+\varepsilon \delta_{T_{T}}}, \quad T_{B}=T_{B}^{S S}, \quad T_{L}=\frac{H_{P}^{S S}\left(\delta_{H}+k_{1}+\varepsilon^{2} \delta_{P}\right)}{k_{-1}}, \quad H_{P}=H_{P}^{S S}, \\
H_{O}=1, \quad R=\frac{\delta_{H}}{\delta_{R}} H_{P}^{S S}, \quad C=0, \quad I_{L}=0, \quad I_{B}=\theta, \quad I_{T}=0,
\end{gathered}
$$

at $t=0$, omitting the somewhat cumbersome form of $T_{B}^{S S}$ and $H_{P}^{S S}$ as before. Rats receive the inhibitor via a food bolus (which must be absorbed from the gut into the bloodstream) or intravenously. Clearly there will be some form of delay in the former, but in the latter this represents an instantaneous introduction to $I_{B}$. The scalings imply that

$$
\text { Scaled total mass } / \text { mole of inhibitor }=\frac{I_{T}}{\varepsilon}+I_{B}+\frac{I_{L}}{v} .
$$

\subsection{Simulations}

In preparation for our time scale analysis, we consider a "typical" evolution first. Figure 3 shows the evolution of each of the ten dimensionless variables; here all the parameters are set to one except $\varepsilon=$ 0.04. The system (2.8a)-(2.2) was solved using Rosenbrock's method implemented in the mathematics package Maple 17. The initial blood concentration of inhibitor is set at $I_{B}(0)=1 / \varepsilon=25$, corresponding to the "high" dose used in the experiments discussed in Section 4. For the remaining variables, we used the pretreatment steady-state concentrations (numerically calculated), namely $T_{T}(0) \approx 1.30, T_{B}(0) \approx$ $1.60, T_{L}(0) \approx 1.96, H_{P}(0) \approx 0.98, H_{O}(0)=1$ and $R(0) \approx 0.98$, and set $I_{T}(0)=I_{L}(0)=C(0)=0$.

We observe that the inhibitor rapidly infiltrates the liver (bottom right) and drives down HPPD (top right) from 1 to about $10^{-5}$; the events leading to this are not clearly seen in the plot, but are discussed in detail in the first part of Section 3. With HPPD concentrations being negligible, tyrosine (top left) builds up fairly rapidly in the liver, then in the blood and, over a longer timescale, in the tissue, all peaking at around the same time at $t \approx 2$ (about 32 hours after the initial dose). Despite the rise in HPPA, homogentistic acid drops to very low concentrations. Following this initial phase, the inhibitor spreads evenly throughout the body and decays allowing HPPD to slowly recover. This continues to about $t \approx 2$ when the inhibitor concentration in the liver collapses as HPPD has built up sufficiently to exhaust supply; the latter eventually drives down HPPA and consequently tyrosine recovers to pretreated 

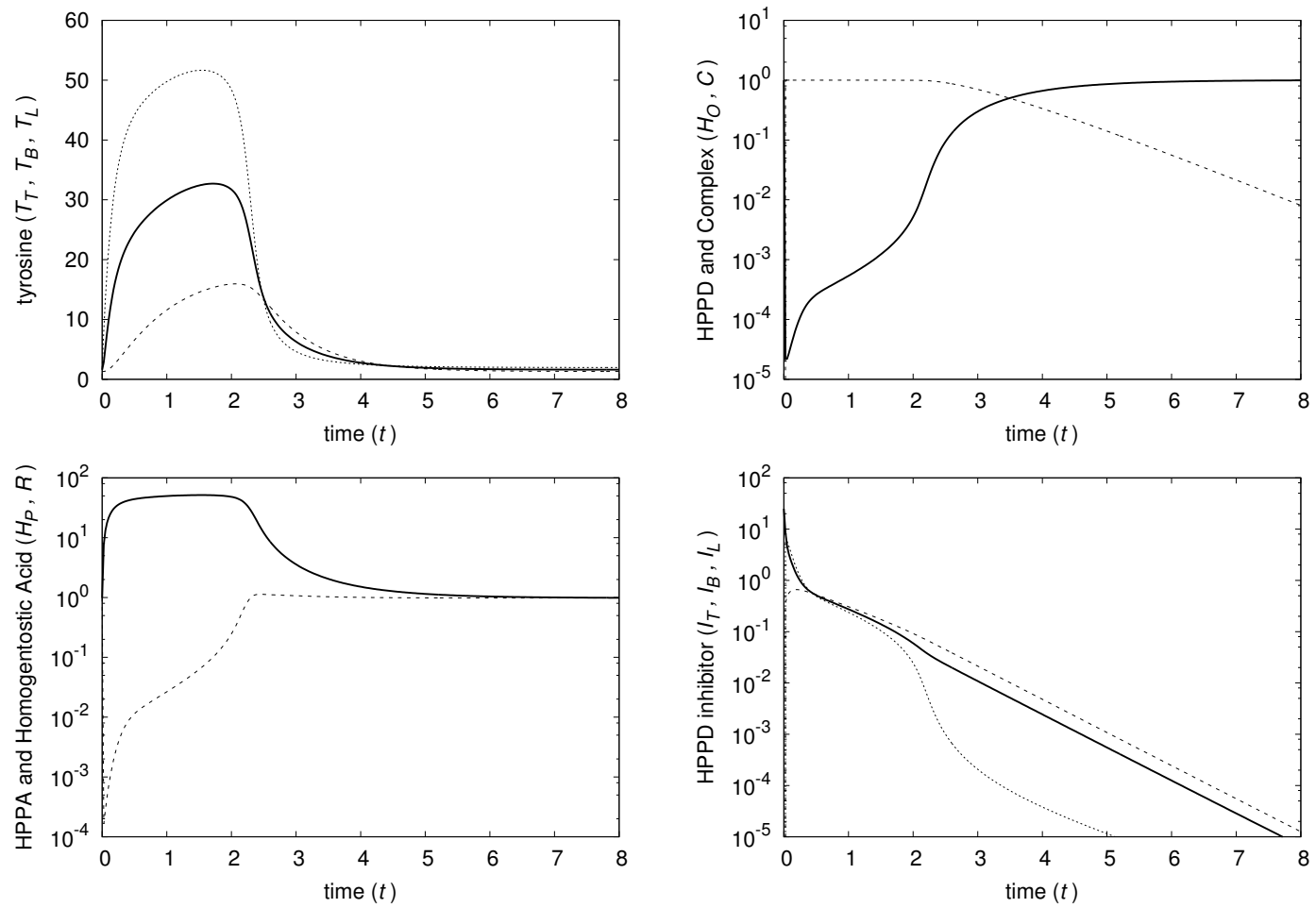

FIG. 3. Plots of the evolution of the dimensionless variables. For the tyrosine (top left) and inhibitor (bottom right), the concentrations in the blood (solid), tissue (dashed) and liver (dotted) are shown. Top right shows the evolution of HPPD (solid) and the complex (dashed) and bottom left shows HPPA (solid) and homogentistic acid (dash). We note from the scalings in Section 2.1, $t=1$ represents about 16 hours. All dimensionless parameters are set to unity except $\varepsilon=0.04$.

concentrations around $t \approx 3$ (about 2 days). Qualitatively speaking, the pattern of behaviour shown in Figure 3 is as to be expected. It is noteworthy however, that the model predicts that homogentistic acid (bottom left) has recovered to pretreated concentrations around $t=2$, reflecting a return to near normal conditions in the liver, despite the animal still being in a high state of tyrosinaemia.

The effect of inhibitor dose on the maximum blood tyrosine $\left(T_{B}\right.$, measurable) and minimum HPPD $\left(H_{O}\right)$ concentrations are shown in Figure 4. The figures appears to show that there is a threshold concentration, say $\theta=\theta^{*}$, between an effective and ineffective dose. When $\theta<\theta^{*}$ there is insufficient inhibitor reaching the liver to drive down HPPD to negligible concentrations, consequently, there is a very little rise in tyrosine. For $\theta>\theta^{*}$, HPPD is exhausted in the liver allowing HPPA, and consequently tyrosine, to rise significantly to a new equilibrium. As the dose increases, HPPD concentrations decrease further (in fact the asymptotic analysis of Section 3 predicts $H_{O_{\min }} \sim 1 / \theta$ ), which means it takes longer to recover and hence allowing more time for tyrosine to build in the blood and tissues. The estimated quantities indicated by the dashed line are discussed in Section 3.2. 

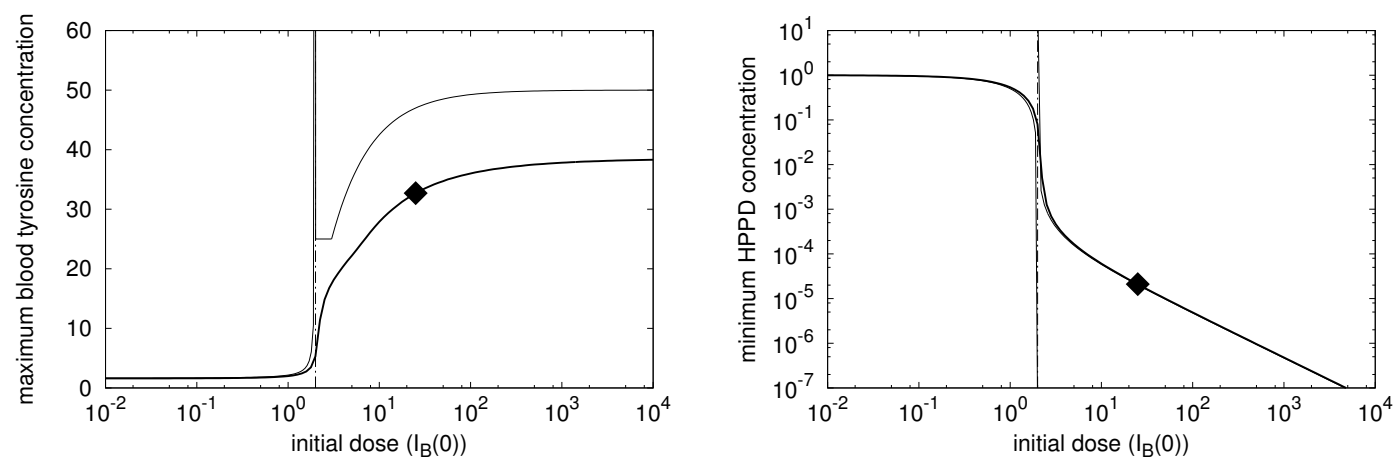

FIG. 4. Figures showing the effect of inhibitor dose on maximum blood tyrosine concentration (left) and on minimum HPPD concentration (right). The corresponds to $\theta=1 / \varepsilon$ (or $\Theta=1$ in Section 3.1) and the vertical dotted line corresponds to $\theta=\theta^{*}$ (here $\theta^{*}=2$ ) marking the switch between ineffective and effective inhibitor dose. The dashed lines are the analytical approximations to these quantities derived in Section 3, and discussed in Section 3.2, specifically that on the left is equation (3.38) and on the right (3.40); note that those for the minimum HPPD are almost superimposed on the numerical solution. All dimensionless parameters are set to unity except for $\varepsilon=0.04$ and $I_{B}(0)=1 / \varepsilon=25$.

\section{Time-scale analysis}

In this Section we exploit the fact the $\varepsilon \ll 1$ to identify key timescales that the model operates over and the processes that are predicted to occur within each timescale. Examination of the data, presented in Section 4, reveals that the maximum tyrosine concentration appears to be about the same for each of the inhibitor doses, which suggests that there is a saturation effect in which the inhibitor has effectively taken out the HPPD, leading to tyrosine to equilibrate to a new, HPPD independent, concentration. Hence we expect for this case that the initial inhibitor concentrations are large compared to the HPPD concentrations and therefore assume $I_{B}(0)=O(1 / \varepsilon)$. With regards to the data, this case is of most interest and hence is analysed in detail in Section 3.1. However, as is observed in Figure 4, the rapid switch, in response to the inhibitor seems to occur when $I_{B}(0)=O(1)$; the analysis for this case is summarised in Section 3.2, where in particular we determine this critical dose in terms of the model parameters.

\subsection{Large initial dose $I_{B}(0)=O(1 / \varepsilon)$}

As stated above, in this Section we analyse the case that the initial inhibitor concentrations are large compared to the HPPD concentrations and hence set

$$
I_{B}(0)=\theta=\frac{\Theta}{\varepsilon}
$$

where constant $\Theta=O(1)$; the initial conditions are to leading order,

$$
\begin{gathered}
T_{T}(0) \sim \frac{S_{1} \alpha_{1} \alpha_{4} \mu_{4}\left(\delta_{H}+k_{1}\right)+\delta_{H} \delta_{T_{T}} k_{-1}\left(\alpha_{1} \mu_{1}+\alpha_{4} \mu_{4}\right)}{\delta_{H} k_{-1}\left(\alpha_{1} \mu_{1} \alpha_{4}+\delta_{T_{T}}\left(\alpha \mu_{1}+\alpha_{4} \mu_{4}\right)\right)}=T_{T_{0}}^{S S} \\
T_{B}(0) \sim \frac{S_{1} \alpha_{1}\left(\delta_{H}+k_{1}\right)\left(\alpha_{4}+\delta_{T_{T}}\right)+\delta_{H} \delta_{T_{T}} k_{-1} \alpha_{4}}{\delta_{H} k_{-1}\left(\alpha_{1} \mu_{1} \alpha_{4}+\delta_{T_{T}}\left(\alpha \mu_{1}+\alpha_{4} \mu_{4}\right)\right)}=T_{B_{0}}^{S S} \\
T_{L}(0) \sim \frac{S_{1}\left(\delta_{H}+k_{1}\right)}{\delta_{H} k_{-1}}=T_{L_{0}}^{S S}, \quad H_{P}(0) \sim \frac{S_{1}}{\delta_{H}}=H_{P_{0}}^{S S}
\end{gathered}
$$




$$
\begin{gathered}
H_{O}(0)=1 \quad R(0) \sim \frac{S_{1}}{\delta_{R}}=R_{0}^{S S} \\
C(0)=0, \quad I_{T}(0)=0, \quad I_{B}(0)=\frac{\Theta}{\varepsilon}, \quad I_{L}(0)=0 .
\end{gathered}
$$

A concentration of $I_{B}(0)=O(1 / \varepsilon)$ means that should the inhibitor instantaneously disperse around the body then (2.9) implies that $I_{i}=O(1)$ throughout. In the course of the analysis we identify sixteen timescales in total, a few describe the main dynamics of the system, whilst the rest are rapid transitional timescales linking the important ones. In the interest of brevity, we summarise the main results within these main timescales in Sections 3.1.1-3.1.6, whereby full details of the asymptotic analysis (for all sixteen timescales) is given in Appendix A. A brief commentary of the sequence of events is presented in Table 3, in which estimates to real time and molecular concentrations are given to help contextualise the analysis.

In the forthcoming analysis, it is customary to indicate the rescaled variables for each of the timescales with some form of notation that uniquely identifies a variable belonging to a particular timescale. In the interest of brevity, this has not been done here. The solutions and discussion on the variables are for the relevant timescale only, unless otherwise stated. The leading order terms for each timescale are indicated with a subscript 0 . The term "negligible" refers to variables being $o(1)$ the size of the pretreated state; this being particularly relevant for HPPD.

3.1.1 Timescale 1: $t=O\left(\varepsilon^{3}\right)$. Initially we look at the short timescale $\left(t \mapsto \varepsilon^{3} t\right)$ where we introduce the scalings

$$
\begin{aligned}
& T_{T} \mapsto T_{T}, \quad T_{B} \mapsto T_{B}, \quad T_{L} \mapsto T_{L}, \quad H_{P} \mapsto H_{P}, \quad H_{O} \mapsto H_{O}, \\
& R \mapsto R, \quad C \mapsto \varepsilon C, \quad I_{T} \mapsto \varepsilon^{2} I_{T}, \quad I_{B} \mapsto \frac{I_{B}}{\varepsilon}, \quad I_{L} \mapsto \varepsilon I_{L} .
\end{aligned}
$$

Applying this to the nondimensional system of equations (2.8), we obtain the leading order solutions

$$
\begin{gathered}
T_{T_{0}}=T_{T_{0}}^{S S}, \quad T_{B_{0}}=T_{B_{0}}^{S S}, \quad T_{L_{0}}=T_{L_{0}}^{S S}, \quad H_{P_{0}}=H_{P_{0}}^{S S}, \\
H_{O_{0}}=1, \quad R_{0}=R_{0}^{S S}, \quad I_{B_{0}}=\Theta .
\end{gathered}
$$

For the remaining variables we note that $d\left(C_{0}+I_{L_{0}}\right) / d t=\alpha_{2} v \Theta$, hence at leading order

$$
\begin{gathered}
I_{T_{0}}=\alpha_{3} \mu_{3} \Theta t, \quad I_{L_{0}}=\frac{v \alpha_{2} \mu_{2} \Theta}{k_{2}}\left(1-e^{-k_{2} t}\right), \\
C_{0}=v \alpha_{2} \mu_{2} \Theta t-\frac{v \alpha_{2} \mu_{2} \Theta}{k_{2}}\left(1-e^{-k_{2} t}\right) .
\end{gathered}
$$

On this initial timescale the inhibitor infiltrates the liver and equilibrates to a level $I_{L} \sim \varepsilon v \alpha_{2} \mu_{2} \Theta / k_{2}$, which is a balance between inhibitor influx and inhibitor reaction with HPPD. Negligible, i.e. $o(1)$, concentrations of HPPD are lost and liver tyrosine produced. The inhibitor is infiltrating tissue at a low, $O(\varepsilon)$, level and the linear accumulation of $I_{T_{0}}$ and $C_{0}$ leads to a change in balance at $t=O(1 / \varepsilon)$.

3.1.2 Timescale 2: $t=O\left(\varepsilon^{2}\right)$. From the large time behaviour of the initial timescale we rescale time such that $t \mapsto \varepsilon^{2} t$ and the variables representing the complex formed from HPPA and HPPD and 
14 of 55

Timescale 1: $t=\mathbf{O}\left(\varepsilon^{3}\right), \mathbf{O}(4)$ seconds

The inhibitor diffuses into the liver and tissue from the blood. Liver concentrations are small though, i.e. it rises to $\mathrm{O}(\varepsilon) \approx 0.01 \mu \mathrm{mole} / \mathrm{ml}$ (tissue concentrations much smaller).

Timescale 2: $t=\mathbf{O}\left(\varepsilon^{2}\right), \mathbf{O}(90)$ seconds

During this timescale HPPD drops rapidly to negligible concentrations. The liver concentrations of inhibitor are still $\mathrm{O}(\varepsilon)$, but this is due to the rapid reaction with HPPD; the inhibitor level eventually rises over a series of intermediate timescales.

Timescale 6: $t=\varepsilon^{2} \eta_{3}+\mathbf{O}\left(\varepsilon^{2}\right), 3+$ minutes

Difficult to be precise on the time in real terms due to the parameters in $\eta_{3}$. However, the inhibitor level in the liver has built up to $\mathrm{O}(1) \approx 0.2 \mu \mathrm{mole} / \mathrm{ml}$. HPPD concentrations are $\mathrm{O}\left(\varepsilon^{2}\right) \approx$ $0.1 \%$ that of the untreated level and homogentistic acid also rockets down to about $0.1 \%$ at the same time.

Timescale 9: $t=\mathbf{O}(\varepsilon), \mathbf{O}(1)$ hour

HPPD is a a minimum level of $\mathrm{O}\left(\varepsilon^{4}\right) \approx 0.0001 \%$ of the healthy level and consequently HPPA is about $20 \times$ its pretreated state. The consequential accumulation of tyrosine in the liver has driven blood concentration to increase by an order of magnitude. However, it has yet to reach its peak due to movement into tissue. The inhibitor is being soaked up by the tissue and its concentration will eventually equilibrate throughout the rat's body,

Timescale 11: $t=\mathbf{O}(1), \mathbf{O}(1)$ day

The tyrosine in the tissue becomes saturated forcing blood concentration to rise further to reach a peak. This continues whilst there is sufficient inhibitor in the liver to maintain negligible HPPD, however, their equilibrated concentration is decaying exponentially. This continues on through Timescale 12 (where inhibitor has dropped by an order of magnitude) and HPPD begins to increase.

Timescale 15: $t=\eta_{12}+\eta_{13}+\mathbf{O}(1), 3+$ days

Following a rapid collapse in liver tyrosine to near normal concentrations over Timescales 13 and 14 , this timescale represents a recovery period throughout the animal. Here, tyrosine concentrations in blood and tissue are in decline (exponentially), being metabolised by HPPD following absorption into the liver. Tyrosine concentration reach their pretreated value in Timescale 16.

Table 3. A list of the major timescales of events following a "large" bolus dose (i.e. $\theta=O(1 / \varepsilon)$ ) of inhibitor. The estimated dimensional values for the timescales and concentrations are included to ease contextualisation. The analytical results for each of these timescales are summarised in Sections 3.1.1-3.1.6 and full details, including the intermediate timescales, are given in Appendix A.

the inhibitor in tissue such that

$$
C \mapsto C, \quad I_{T} \mapsto \varepsilon I_{T} .
$$

When applied to equations (2.8), this leaves the leading order solutions for tyrosine and the inhibitor in the tissue and blood unchanged

$$
T_{T_{0}}=T_{T_{0}}^{S S}, \quad T_{B_{0}}=T_{B_{0}}^{S S}, \quad I_{B_{0}}=\Theta, \quad I_{T_{0}}=\alpha_{3} \mu_{3} \Theta t
$$


while the solutions for the complex, HPPD and its inhibitor in the liver are now

$$
C_{0}=v \alpha_{2} \mu_{2} \Theta t, \quad H_{O_{0}}=1-v \alpha_{2} \mu_{2} \Theta t, \quad I_{L_{0}}=\frac{v \alpha_{2} \mu_{2} \Theta}{k_{2}\left(1-\Theta v \alpha_{2} \mu_{2} t\right)} .
$$

The remaining variables are the solutions of differential equations that seem not to be analytically solvable, namely

$$
\begin{aligned}
& \frac{d T_{L_{0}}}{d t}=S_{1}+k_{1} H_{P_{0}}-k_{-1} T_{L_{0}}, \\
& \frac{d H_{P_{0}}}{d t}=-k_{1} H_{P_{0}}+k_{-1} T_{L_{0}}-\delta_{H}\left(1-v \alpha_{2} \mu_{2} \Theta t\right) H_{P_{0}}, \\
& \frac{d R_{0}}{d t}=\delta_{H}\left(1-v \alpha_{2} \mu_{2} \Theta t\right) H_{P_{0}}-\delta_{R} R_{0} .
\end{aligned}
$$

On this timescale we find that HPPD concentrations $\left(H_{O_{0}}\right)$ "collapse" and liver inhibitor concentrations $\left(I_{L_{0}}\right)$ "explode" in finite time, specifically as $t \rightarrow \eta_{3}(\varepsilon)^{-}$, where $\eta_{3}(0)=1 / v \alpha_{2} \mu_{2} \Theta$, whilst all other variables maintain their order.

3.1.3 Timescale 6: $t=\varepsilon^{2} \eta_{3}(\varepsilon)+O\left(\varepsilon^{2}\right)$. After several rapid transitional timescales, we now move to Timescale 6 where time is rescaled such that $t \mapsto \varepsilon^{2} \eta_{3}(\varepsilon)+\varepsilon^{2} t$. Most variables retain the scalings of timescale two except those for HPPA and the inhibitor in liver which are rescaled such that

$$
H_{O} \mapsto \varepsilon^{3} H_{O}, \quad I_{L} \mapsto I_{L} .
$$

At leading order the following solutions remain as they were at timescale two

$$
T_{T_{0}}=T_{T_{0}}^{S S}, \quad T_{B_{0}}=T_{B_{0}}^{S S}, \quad I_{B_{0}}=\Theta
$$

whilst

$$
C_{0}=1
$$

and

$$
\begin{aligned}
& I_{T_{0}}=\frac{\alpha_{3} \mu_{3}}{\alpha_{2} \mu_{2} v}+\alpha_{3} \mu_{3} \Theta t, I_{L_{0}}=v \alpha_{2} \mu_{2} \Theta t, H_{O_{0}}=\frac{\delta_{O}+k_{-2}}{k_{2} v \alpha_{2} \mu_{2} \Theta t}, R_{0}=R_{0}^{[2]}\left(\eta_{3}\right) e^{-\delta_{R} t} \\
& H_{P_{0}}=H_{P_{0}}^{[2]}\left(\eta_{3}\right)+\frac{k_{-1} S_{1}}{k_{1}+k_{-1}} t+\frac{\left(1-e^{-\left(k_{1}+k_{-1}\right) t}\right)}{k_{1}+k_{-1}}\left(k_{1} T_{L_{0}}^{[2]}\left(\eta_{3}\right)-k_{-1} H_{P_{0}}^{[2]}\left(\eta_{3}\right)-\frac{k_{-1} S_{1}}{k_{1}+k_{-1}}\right) \\
& T_{L_{0}}=T_{L_{0}}^{[2]}\left(\eta_{3}\right)+\frac{k_{1} S_{1}}{k_{1}+k_{-1}} t-\frac{\left(1-e^{-\left(k_{1}+k_{-1}\right) t}\right)}{k_{1}+k_{-1}}\left(k_{1} T_{L_{0}}^{[2]}\left(\eta_{3}\right)-k_{-1} H_{P_{0}}^{[2]}\left(\eta_{3}\right)-\frac{k_{-1} S_{1}}{k_{1}+k_{-1}}\right)
\end{aligned}
$$

where the superscript [2] refers to the solution in Timescale 2. The inhibitor is infiltrating into the tissue, whilst the inhibitor in the liver $\left(I_{L_{0}}\right)$, HPPA $\left(H_{P_{0}}\right)$ and liver tyrosine concentration $\left(T_{L_{0}}\right)$ increases and HPPD $\left(H_{O_{0}}\right)$ concentration decreases. Blood tyrosine is relatively unchanged $\left(T_{B_{0}}\right)$, however, the homogentisic acid $(R)$ and downstream products $(P)$ drop rapidly. 
16 of 55

3.1.4 Timescale 9: $t=O(\varepsilon)$. For this timescale we write $t \mapsto \varepsilon t$ and find that, since Timescale 6, the following scalings have changed

$$
\begin{gathered}
T_{B} \mapsto \frac{T_{B}}{\varepsilon}, \quad T_{L} \mapsto \frac{T_{L}}{\varepsilon}, \quad H_{P} \mapsto \frac{H_{P}}{\varepsilon}, \quad H_{O} \mapsto \varepsilon^{4} H_{O}, \\
R \mapsto \varepsilon^{3} R, \quad I_{T} \mapsto I_{T}, \quad I_{L} \mapsto \frac{I_{L}}{\varepsilon} .
\end{gathered}
$$

The leading order solution for the complex remains as it was at Timescale 6

$$
C_{0}=1 \text {. }
$$

The equations for tyrosine and HPPA decouple and are solutions to linear second order differential equations, given by

$$
\begin{aligned}
T_{B_{0}} & =\frac{S_{1}}{v \alpha_{4} \mu_{4}}\left(1+E_{T}^{+}(t)-E_{T}^{-}(t)\right), \\
T_{T_{0}} & =T_{T_{0}}^{S S}+\frac{S_{1}}{v}\left(t-\left(E_{T}^{+}(0)-E_{T}^{+}(t)\right)+\left(E_{T}^{-}(0)-E_{T}^{-}(t)\right)\right), \\
T_{L_{0}} & =\frac{S_{1}\left(\alpha_{1} \mu_{1}+\alpha_{4} \mu_{4}\right)}{v \alpha_{1} \alpha_{4} \mu_{4}}+\frac{S_{1}}{v \alpha_{1} \alpha_{4} \mu_{4}}\left(\omega_{\alpha \mu}^{-} E_{T}^{+}(t)+\omega_{\alpha \mu}^{+} E_{T}^{-}(t)\right), \\
H_{P_{0}} & =\frac{S_{1} k_{1}\left(\alpha_{1} \mu_{1}+\alpha_{4} \mu_{4}\right)}{k_{-1} v \alpha_{1} \alpha_{4} \mu_{4}}+\frac{S_{1} k_{1}}{k_{-1} v \alpha_{1} \alpha_{4} \mu_{4}}\left(\omega_{\alpha \mu}^{-} E_{T}^{+}(t)+\omega_{\alpha \mu}^{+} E_{T}^{-}(t)\right),
\end{aligned}
$$

where $E_{T}^{ \pm}(t)=\omega^{+} \omega^{-} e^{\omega^{ \pm} t} / \omega^{ \pm}\left(\omega^{+}-\omega^{-}\right)$and $\omega_{\alpha \mu}^{ \pm}=\alpha_{1} \mu_{1}+\alpha_{4} \mu_{4}+\omega^{ \pm}$, where

$$
\omega^{ \pm}=-\frac{1}{2}\left(\frac{k_{1} v \alpha_{1}}{k_{1}+k_{-1}}+\alpha_{1} \mu_{1}+\alpha_{4} \mu_{4}\right) \pm \frac{1}{2}\left(\left(\frac{k_{1} v \alpha_{1}}{k_{1}+k_{-1}}+\alpha_{1} \mu_{1}-\alpha_{4} \mu_{4}\right)^{2}+4 \alpha_{1} \mu_{1} \alpha_{4} \mu_{4}\right)^{1 / 2}
$$

noting that $\omega^{-}<\omega^{+}<0$ and hence $E_{T}^{ \pm}(t) \rightarrow 0$ as $t \rightarrow \infty$. These results show that tyrosine concentration is rising everywhere, however, the seepage into tissue means that an equilibrium is eventually reached in the liver and blood, namely

$$
T_{B_{0}} \sim \frac{S_{1}}{v \alpha_{4} \mu_{4}}=T_{B_{\infty}}^{[9]}, \quad T_{L_{0}} \sim \frac{S_{1}\left(\alpha_{1} \mu_{1}+\alpha_{4} \mu_{4}\right)}{v \alpha_{1} \alpha_{4} \mu_{4}}=T_{L_{\infty}}^{[9]}
$$

We note, these equilibrium values are a temporary maximum level, which are superseded when tyrosine saturates in the tissue (Timescale 11). This indicates that there is a two stage "jumping" of tyrosine concentration; though not observable in the data, or in the simulations in Figure 3, they do become apparent for smaller $\varepsilon$ (Figure 5, top right).

Similarly to the tyrosine equations, the equations for the inhibitor also decouple into a linear second order differential equations, namely

$$
\begin{gathered}
I_{L_{0}}=\Theta v \alpha_{2} \mu_{2}\left(E_{I}^{+}(t)-E_{I}^{-}(t)\right), \quad I_{B_{0}}=\Theta\left(\rho_{\alpha \mu}^{+} E_{I}^{-}(t)-\rho_{\alpha \mu}^{-} E_{I}^{+}(t)\right) \\
I_{T_{0}}=\Theta\left(1+\rho_{\alpha \mu}^{-}\left(\rho_{\alpha \mu}^{+}+v \alpha_{2}\right) E_{I}^{+}(t)-\rho_{\alpha \mu}^{+}\left(\rho_{\alpha \mu}^{-}+v \alpha_{2}\right) E_{I}^{-}(t)\right)
\end{gathered}
$$

where $E_{I}^{ \pm}(t)=e^{\rho^{ \pm} t} /\left(\rho^{+}-\rho^{-}\right)$and $\rho_{\alpha \mu}^{ \pm}=\alpha_{2} \mu_{2}+\alpha_{3} \mu_{3}+\rho^{ \pm}$, where

$$
\left.\rho^{ \pm}=-\frac{\left(\alpha_{3} \mu_{3}+\alpha_{2}\left(\mu_{2}+v\right)\right)}{2} \pm \frac{1}{2}\left(\alpha_{3} \mu_{3}+\alpha_{2}\left(\mu_{2}+v\right)\right)^{2}-4 v \alpha_{2} \alpha_{3} \mu_{3}\right)^{1 / 2}
$$


noting that $\rho^{-}<\rho^{+}<0$ and hence $E_{I}^{ \pm}(t) \rightarrow 0$ as $t \rightarrow \infty$. For brevity, we express the leading solutions of the remaining variables as follows

$$
H_{O_{0}}=\frac{\delta_{O}+k_{-2}}{k_{2}} \frac{1}{I_{L_{0}}}, \quad R_{0}=\frac{\delta_{H}\left(\delta_{O}+k_{-2}\right)}{\delta_{R} k_{2}} \frac{T_{L_{0}}}{I_{L_{0}}}
$$

Inhibitor concentration in the blood $\left(I_{B_{0}}\right)$ and eventually in the liver $\left(I_{L_{0}}\right)$ declines as it infiltrates into tissue acting as a inhibitor sink, rising to a maximum level of $I_{T_{0}} \sim \Theta$. As liver inhibitor concentration decline, HPPD and homogentisic acid start to rise.

3.1.5 Timescale 11: $t=O(1)$. On this timescale the following new scalings have been introduced since Timescale 9 to reflect that $I_{B}=O(1)$ and is no longer large:

$$
T_{T} \mapsto \frac{T_{T}}{\varepsilon}, \quad H_{O} \mapsto \varepsilon^{3} H_{O}, \quad R \mapsto \varepsilon^{2} R, \quad I_{B} \mapsto I_{B}, \quad I_{L} \mapsto I_{L} .
$$

At leading order, the equations representing the inhibitor can be solved to give

$$
I_{T_{0}}=\Theta e^{-\delta_{I_{t}} t}, \quad I_{B_{0}}=\frac{\Theta}{\mu_{3}} e^{-\delta_{I_{t}} t}, \quad I_{L_{0}}=\frac{\Theta \mu_{2}}{\mu_{3}} e^{-\delta_{I_{t}} t},
$$

and those for the complex concentration leads and

$$
C_{0}=\frac{\delta_{O}}{\delta_{C}}+\left(1-\frac{\delta_{O}}{\delta_{C}}\right) e^{-\delta_{C} t}
$$

and for HPPD,

$$
H_{O_{0}}=\frac{\mu_{3}}{k_{2} \mu_{2} \Theta}\left(\delta_{O}\left(1+\frac{k_{-2}}{\delta_{C}}\right)+\left(1-\frac{\delta_{O}}{\delta_{C}}\right) e^{-\delta_{C} t}\right) e^{\delta_{I_{T}} t}
$$

The solutions for tyrosine and HPPA are

$$
\begin{aligned}
T_{T_{0}} & =\frac{S_{1}}{v \delta_{T_{T}}}\left(1-e^{-\delta_{T_{T}} t}\right), \\
T_{B_{0}} & =\frac{S_{1}}{v \alpha_{4} \mu_{4}}+\frac{S_{1}}{v \delta_{T_{T}} \mu_{4}}\left(1-e^{-\delta_{T_{T}} t}\right), \\
T_{L_{0}} & =\frac{S_{1}\left(\alpha_{1} \mu_{1}+\alpha_{4} \mu_{4}\right)}{v \alpha_{1} \alpha_{4} \mu_{4}}+\frac{S_{1} \mu_{1}}{v \delta_{T_{T}} \mu_{4}}\left(1-e^{-\delta_{T_{T}} t}\right), \\
H_{P_{0}} & =\frac{S_{1} k_{-1}\left(\alpha_{1} \mu_{1}+\alpha_{4} \mu_{4}\right)}{k_{1} v \alpha_{1} \alpha_{4} \mu_{4}}+\frac{S_{1} k_{-1} \mu_{1}}{k_{1} v \delta_{T_{T}} \mu_{4}}\left(1-e^{-\delta_{T_{T}} t}\right) .
\end{aligned}
$$

Finally, we obtain for the homogentisic acid $R_{0}=\delta_{H} H_{O} H_{P} / \delta_{R}$.

Reflecting on these results, we note that tissue tyrosine concentration is matching that of the blood and liver and leads to further build up of tyrosine throughout; HPPA suitably adjusts to this build up. Inhibitor has equilibrated and is decaying naturally (note the vast majority of the inhibitor is in tissue and its kinetics dominate the overall decay rate). HPPD and homogentisic acid are increasing, but 
18 of 55

concentrations are still very small, and the complex adjusts to a new equilibrium as HPPD production and the complex's natural decay balance out. The large time solutions are

$$
\begin{gathered}
T_{T_{0}} \sim \frac{S_{1}}{v \delta_{T_{T}}}=T_{T_{\infty}}^{[11]}, \quad T_{L_{0}} \sim \frac{S_{1}}{v \mu_{4}}\left(\frac{\mu_{1}}{\delta_{T_{T}}}+\frac{\mu_{1}}{\alpha_{4}}+\frac{\mu_{4}}{\alpha_{1}}\right)=T_{L_{\infty}}^{[11]}, \\
H_{P_{0}} \sim \frac{S_{1} k_{-1}}{k_{1} v \mu_{4}}\left(\frac{\mu_{1}}{\delta_{T_{T}}}+\frac{\mu_{1}}{\alpha_{4}}+\frac{\mu_{4}}{\alpha_{1}}\right)=H_{P_{\infty}}^{[11]}, \quad C_{0}=\frac{\delta_{O}}{\delta_{C}},
\end{gathered}
$$

and, of particular interest, is the leading order maximum blood tyrosine level, namely

$$
T_{B_{0}} \sim \frac{S_{1}}{v \mu_{4}}\left(\frac{1}{\alpha_{4}}+\frac{1}{\delta_{T_{T}}}\right)=T_{B_{\infty}}^{[11]},
$$

as $t \rightarrow \infty$, where we note in particular its independence to the initial inhibitor concentration $\Theta$. These expansions become invalid when $I_{L_{0}}=O(1 / \varepsilon)$, i.e. when $t=\delta_{I_{T}}^{-1} \ln (1 / \varepsilon)+O(1)$.

Numerical simulations aimed at testing the accuracy of the approximations revealed that the large time value for $T_{B_{0}}$ turns out not to predict well the peak of blood tyrosine level using $\varepsilon \approx 0.04$ (about $40-50 \%$ error); see Figure 4, particularly for $\theta>\theta^{*}$. When $t \sim \delta_{I_{T}}^{-1} \ln (1 / \varepsilon)$, we have to leading order $T_{B} \sim\left(T_{B_{0}}^{[11]}+O\left(\varepsilon^{\delta_{T_{T}} / \delta_{I_{T}}}\right)\right) / \varepsilon$; since the parameters are set to one, the error is about -1 of the predicted maximum, $T_{B_{0}}^{[11]} / \varepsilon$. However, analysis of the correction term, using the expansions $T_{B} \sim T_{B_{0}}+\varepsilon T_{B_{1}}$ etc., reveals that $T_{B_{1}} \rightarrow T_{B_{1_{\infty}}}^{[11]}$, where

$$
T_{B_{1} \infty}^{[11]} \sim-\frac{S_{1}\left(\alpha_{4}+\delta_{T_{T}}\right)\left(\left(\delta_{T_{L}} k_{1}+\delta_{P} k_{-1}\right)\left(\alpha_{1} \mu_{1} \alpha_{4}+\delta_{T_{T}}\left(\alpha_{1} \mu_{1}+\alpha_{4} \mu_{4}\right)\right)+k_{1} \delta_{T_{B}} v \alpha_{1}\left(\alpha_{4}+\delta_{T_{T}}\right)\right)}{k_{1} v^{2} \delta_{T_{T}}^{2} \alpha_{1} \alpha_{4}^{2} \mu_{4}^{2}}+\frac{1}{\mu_{4}},
$$

as $t \rightarrow \infty$. Here, $T_{B_{1}}^{[11]}$ consists of several products of sums, so if, for example, all parameters are set to one, then $T_{B_{1}}^{[11]} \approx-15$ and makes a significant contribution to the estimated maximum value of $T_{B}$ when the modestly small $\varepsilon \approx 0.04$ is used. However, in the limit $\varepsilon \rightarrow 0$ this issue is not a problem (see Figure 5 , top right).

Much of the data of blood tyrosine and inhibitor concentrations correspond to this timescale. The exponential decay of $I_{B_{0}}$ predicted in equation (3.12) will form the basis for fitting with the inhibitor data, whilst a modified form of (3.16) will be used to fit part of the blood tyrosine data.

3.1.6 Timescale 15: $t=\eta_{12}(\varepsilon)+\eta_{13}(\varepsilon)+O(1)$. Where $\eta_{12}(\varepsilon)=\delta_{I_{T}}^{-1} \ln (1 / \varepsilon)$ and $\eta_{13}(\varepsilon)=$ $O(1)$ defined so that $\eta_{13}(0)=\delta_{I_{T}}^{-1} \ln \left[\Theta v \alpha_{2} \mu_{2} \alpha_{3} \delta_{I_{T}} / \delta_{O}\left(\alpha_{2} \mu_{2} \alpha_{3}+\delta_{I_{T}}\left(\alpha_{2} \mu_{2}+\alpha_{3} \mu_{3}\right)\right)\right]$. Timescale 12 sees the continuation of inhibitor decline and slow HPPD recovery for an $O(1)$ time, until there is a rather sudden collapse of inhibitor concentration in the liver, namely $O(\varepsilon) \rightarrow O\left(\varepsilon^{3}\right)$, as HPPD production rate finally overtakes inhibitor absorption from the blood reservoir and recovers to near its pretreated level (Timescales 13 and 14). Furthermore, the rapid rise in HPPD leads to a rapid collapse in liver tyrosine, causing a rapid drop in blood tyrosine from its peak $\left(T_{B_{\infty}}^{[11]}\right)$ to a new level $\left(T_{B_{\infty}}^{[14]}\right.$, defined below), though still much higher than the pretreated concentration due to the tyrosine in tissue acting as the main reservoir. We note further, that since the initial inhibitor concentration $\theta=\Theta / \varepsilon$, with 
$\Theta=O(1)$, is sufficient to eliminate HPPD, the only other significant effect it has is in delaying the onset of Timescale 13 and consequently the current one.

Writing $t \mapsto \eta_{12}(\varepsilon)+\eta_{13}(\varepsilon)+t$, the following rescalings have changed since Timescale 11,

$$
T_{L} \mapsto T_{L}, \quad H_{P} \mapsto H_{P}, \quad H_{O} \mapsto H_{O}, \quad I_{L} \mapsto \varepsilon^{3} I_{L}
$$

We find that blood and tissue tyrosine are dropping according to

$$
T_{T_{0}}=\frac{S_{1}}{v \delta_{T_{T}}} e^{-\Omega_{T}^{[15]} t}, \quad T_{B_{0}}=T_{B_{\infty}}^{[14]} e^{-\Omega_{T}^{[15]} t}
$$

where $T_{B_{\infty}}^{[14]}=S_{1} \alpha_{4} / v \delta_{T_{T}}\left(\alpha_{1} \mu_{1}+\alpha_{4} \mu_{4}\right)$ and

$$
\Omega_{T}^{[15]}=\frac{\alpha_{1} \mu_{1} \alpha_{4}+\delta_{T_{T}}\left(\alpha_{1} \mu_{1}+\alpha_{4} \mu_{4}\right)}{\alpha_{1} \mu_{1}+\alpha_{4} \mu_{4}}
$$

Defining

$$
\phi_{O}(t)=\Omega_{I}^{[15]} e^{-\delta_{O} t}-\delta_{O} e^{-\Omega_{I}^{[15]} t}, \quad \phi_{C}(t)=\Omega_{I}^{[15]} e^{-\delta_{C} t}-\delta_{C} e^{-\Omega_{I}^{[15]} t}
$$

where $\Omega_{I}^{[15]}=\left(\alpha_{2} \mu_{2} \alpha_{3}+\delta_{I_{T}}\left(\alpha_{2} \mu_{2}+\alpha_{3} \mu_{3}\right)\right) /\left(\alpha_{2} \mu_{2}+\alpha_{3} \mu_{3}\right)$, noting that $\phi_{i}(t) \rightarrow 0$ as $t \rightarrow \infty$, then HPPD, HPPA, liver tyrosine and homogentisic acid return to their pretreated concentrations according to

$$
\begin{aligned}
H_{O_{0}} & =1-\frac{\phi_{O}(t)}{\phi_{O}(0)} \\
H_{P_{0}} & =H_{P_{0}}^{S S}+\frac{S_{1} \phi_{O}(t)+v \alpha_{1} \mu_{1} \phi_{O}(0) T_{B_{\infty}}^{[14]} e^{-\Omega_{T}^{[15]} t}}{\delta_{H}\left(\phi_{O}(0)-\phi_{O}(t)\right)} \\
T_{L_{0}} & =T_{L_{0}}^{S S}+\frac{S_{1} k_{1} \phi(t)+v \alpha_{1} \mu_{1} T_{B_{\infty}}^{[14]} e^{-\Omega_{T}^{[15]} t}\left(\phi_{O}(0)\left(\delta_{H}+k_{1}\right)-\delta_{H} \phi_{O}(t)\right)}{\delta_{H} k_{-1}\left(\phi_{O}(0)-\phi_{O}(t)\right)} \\
R_{0} & =R_{0}^{S S}-\frac{\delta_{H}}{\delta_{R}}\left(\frac{\phi_{O}(t)}{\phi_{O}(0)} H_{P_{0}}^{S S}+\left(1-\frac{\phi_{O}(t)}{\phi_{O}(0)}\right)\left(\frac{S_{1} \phi_{O}(t)+v \alpha_{1} \mu_{1} \phi_{O}(0) T_{B_{\infty}}^{[14]} e^{-\Omega_{T}^{[15]} t}}{\delta_{H}\left(\phi_{O}(0)-\phi_{O}(t)\right)}\right)\right) .
\end{aligned}
$$

The rising concentration of HPPD accelerates absorption and degradation of the inhibitor in the liver from the blood and tissue, thus the inhibitor related variables decay

$$
\begin{gathered}
I_{T_{0}}=\frac{\delta_{O}\left(\alpha_{2} \mu_{2}+\alpha_{3} \mu_{3}\right)}{v \alpha_{2} \mu_{2} \alpha_{3}} e^{-\Omega_{I}^{[15]} t}, \quad I_{B_{0}}=\frac{\delta_{O}}{v \alpha_{2} \mu_{2}} e^{-\Omega_{I}^{[15]} t}, \quad C=\frac{\delta_{O}}{\delta_{C}} \frac{\phi_{C}(t)}{\phi_{C}(0)} \\
I_{L_{0}}=\frac{\delta_{O} e^{-\Omega_{I}^{[15]} t}+k_{-2} \delta_{O} \phi_{C}(t) / \delta_{C} \phi_{C}(0)}{k_{2}\left(1-\phi_{O}(t) / \phi_{O}(0)\right)}
\end{gathered}
$$

All that remains is for the blood and tissue tyrosine to return to their pretreated concentrations. This occurs in Timescale 16, where $t \mapsto \eta_{12}(\varepsilon)+\eta_{13}(\varepsilon)+\eta_{16}(\varepsilon)+t$ (here, $\eta_{16}(\varepsilon)=\ln (1 / \varepsilon) / \Omega_{T}^{[15]}$ ) and 
20 of 55

both $T_{T}$ and $T_{B}$ are $O(1)$ satisfying

$$
T_{T_{0}}=T_{T_{0}}^{S S}+\frac{S_{1}}{v \delta_{T_{T}}} e^{-\Omega_{T}^{[15]} t}, \quad T_{B_{0}}=T_{B_{0}}^{S S}+T_{B_{\infty}}^{[14]} e^{-\Omega_{T}^{[15]} t}
$$

hence tyrosine concentration tend to the pretreated state to leading order as $t \rightarrow \infty$.

3.1.7 Comparison with numerical solutions Figure 5 shows a selection of comparisons between the numerical solutions for the full-system and composites of the leading-order asymptotic terms. The composite solutions are derived by adding successive timescale solutions and subtracting the common term; although in the cases shown, most of contributions from the successive time-scales cancel each other out. For the plots in Figure 5, the solution for blood tyrosine, $T_{B}^{\text {comp }}$, complex $C^{\text {comp }}$ and blood nitisone $I_{B}^{c o m p}$ are, in their most reduced form,

$$
\begin{aligned}
& T_{B}^{\text {comp }}=H\left(t^{[14]}-t\right)\left(T_{B_{0}}^{S S}+T_{B_{0}}^{[9]}+T_{B_{0}}^{[11]}-T_{B_{\infty}}^{[9]}\right)+H\left(t-t^{[14]}\right)\left(T_{B_{0}}^{[14]}+T_{B_{0}}^{[16]}-T_{B_{\infty}}^{[14]}\right), \\
& C^{\text {comp }}=H\left(t^{[2]}-t\right)\left(C_{0}^{[1]}+C_{0}^{[2]}-C_{\infty}^{[1]}\right)+H\left(t-t^{[2]}\right)\left(C_{0}^{[11]}+H\left(t-t^{[14]}\right)\left(C_{0}^{[15]}-C_{0}^{[11]}\right)\right), \\
& I_{B}^{c o m p}=H\left(t^{[14]}-t\right)\left(I_{B_{0}}^{[9]}+I_{B_{0}}^{[10]}+I_{B_{0}}^{[11]}+I_{B_{0}}^{[12]}-I_{B_{\infty}}^{[9]}-I_{B_{\infty}}^{[10]}-I_{B_{\infty}}^{[11]}\right)+H\left(t-t^{[14]}\right) I_{B_{0}}^{[15]},
\end{aligned}
$$

where $*_{0}^{[i]}$, with $*=\left\{T_{B}, C, I_{B}\right\}$, is the leading-order solution of $*$ for timescale $i, *_{\infty}^{[i]}$ is the corresponding large time limit, $t^{[2]}=\varepsilon^{2} \eta_{3}$ and $t^{[14]}=\eta_{12}(\varepsilon)+\eta_{13}(0)$, and $H(z)$ is the Heaviside step function such that $H(z)=0$ for $z<0$ and $H(z)=1$ for $z \geq 1$. The step-functions are used as a convenient way of eliminating the complexities in the intermediate timescales around $t=t^{[2]}$ and $t^{[14]}$, whereby the solutions in intermediate timescales provide jump conditions for the composite solutions either side of them (although the only discontinuity here is $T_{B}$ at $t^{[14]}$ ); we note that the composite solutions (3.34)-(3.36) are asymptotically valid as $\varepsilon \rightarrow 0$. For HPPD, $H_{O}$, and liver drug concentration, $I_{L}$, it is not possible to construct a likely looking composite solution using leading order approximations, due to the tails of the solutions for one timescale significantly encroaching on the solutions of the neighbouring ones when $\varepsilon$ is modestly small; we hence show results for individual timescales (open shapes) and composites of a short sequence of timescales (solid shapes).

Formally, the asymptotic analysis only applies in the limit $\varepsilon \rightarrow 0$, nevertheless the figures shows that there is generally good agreement between the analytical and numerical solutions for $\varepsilon=0.04$. For $C, I_{B}$ and $I_{T}$ (not shown), the composite solutions agree very well across the entire time interval, whilst for $H_{O}, I_{L}$ and $R$ (not shown) the approximations agree well within their own timescale. The solutions for $T_{B}, T_{T}, T_{L}$ and $H_{P}$ have similar qualitative profiles, for which only $T_{B}$ is shown for brevity. For the parameter set used, there is a significant disparity around where $T_{B}$ reaches its maximum (as seen in Figure 4), the reasons for which being discussed in Section 3.1.5; we note there are similar disparities for $T_{T}, T_{L}$ and $H_{P}$ as well. However, as expected, the agreement can be much improved by reducing $\varepsilon$, as can be seen for $\varepsilon=0.01$ case in the top right plot. We also note, that the two stage accumulation of $T_{B}$ during Timescales 9 and 11 is observable in this plot.

\subsection{Moderate initial dose $I_{B}(0)=O(1)$}

The analysis for the case $I_{B}(0)=\theta=O(1)$ follows the same lines as that described in Section 3.1 and we omit all the details for brevity. This analysis is motivated by the results shown in Figure 4 where 

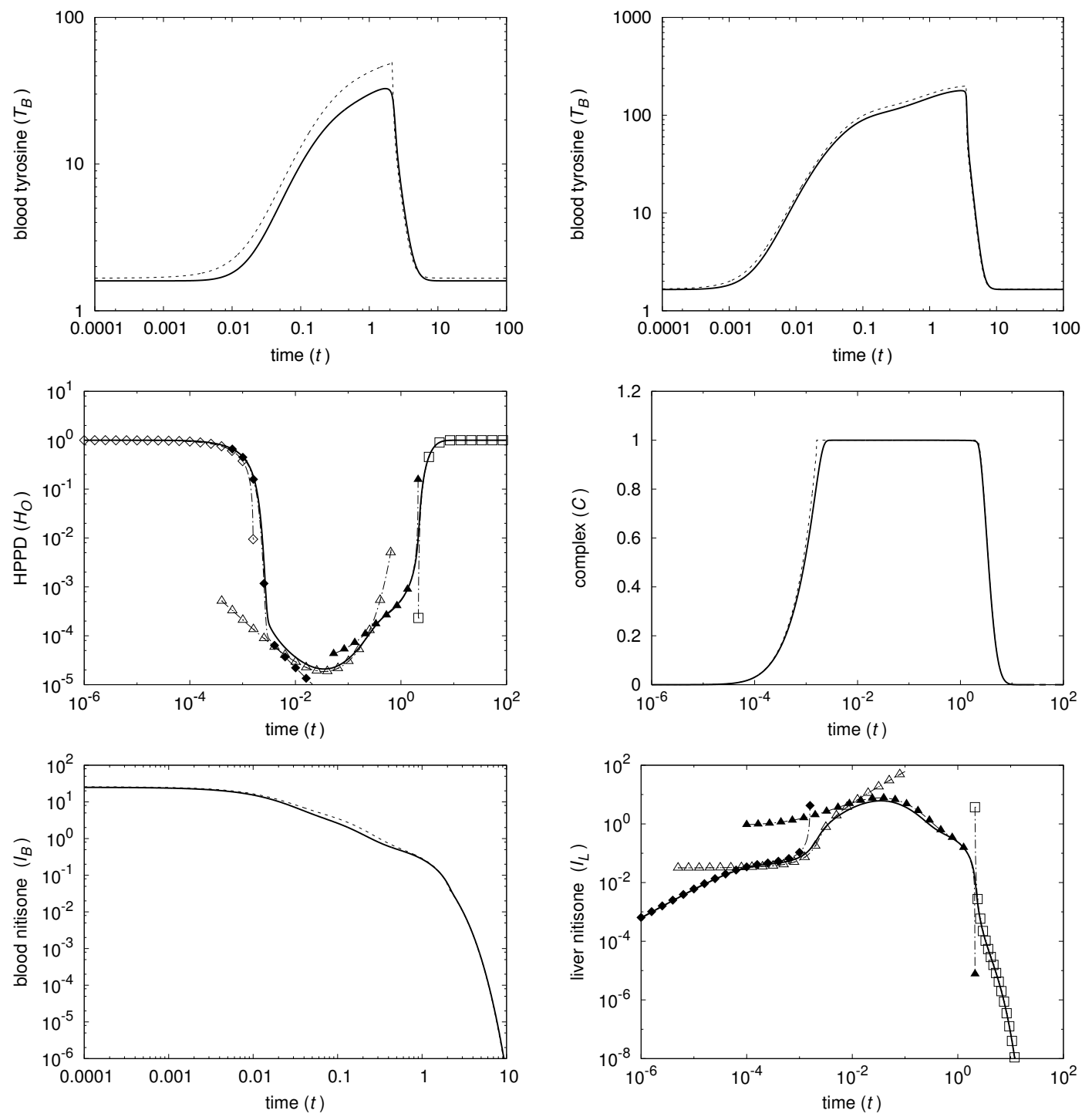

FIG. 5. Figures comparing the numerical solutions of the full system (solid lines) with asymptotic solutions (dashed or dashed with symbols). The top two figures compares the composite solution of $T_{B}$, as given by (3.34), for the cases for $\varepsilon=0.04$ (top left) and $\varepsilon=0.01$ (top right), respectively. Middle left compares the asymptotic and numerical solutions of $H_{O}$ at various timescales: timescales $2(\diamond)$, composite of timescales 3-8 $(\diamond)$, timescale $9(\Delta), 10-12(\boldsymbol{\Delta})$ and $15(\square)$. Middle right and bottom left show full composite solutions of $C$ and $I_{B}$, respectively. Bottom right shows the solutions for $I_{L}$ at timescales 1-2 $(\diamond), 3(\triangle), 9-12(\Delta)$ and 15 (ם). All dimensionless parameters are set to unity with $\theta=1 / \varepsilon$ (or $\Theta=1$ ) and $\varepsilon=0.04$.

there appears to be a critical inhibitor concentration, denoted $\theta^{*}$, beyond which the inhibitor is effective at substantially reducing HPPD concentration, with a consequential rise in tyrosine concentration. Undertaking the time-scale analysis for this case reveals that the critical time point which results in the divergence in inhibitor effectiveness is in the third timescale $(t=O(\varepsilon))$ of this analysis, whereby the 
"critical concentration" is revealed, namely

$$
\theta^{*}=\frac{1}{v}\left(1+\frac{\alpha_{3} \mu_{3}}{\alpha_{2} \mu_{2}}\right)
$$

A remarkable feature of this critical concentration is that it is independent of the reaction kinetics at leading order and is governed by the relative volumes of blood and liver $(v)$ and the transport and partitioning parameters of the inhibitor across the liver/blood/tissue phases. This is actually due, according to the model, to the reactions being so rapid that the limiting factor in inhibitor effectiveness is delivery to the liver.

Further to this, the analysis enables us to derive a formula for the maximum amount of tyrosine in the blood. The details are technical and there are several cases depending on the relative sizes of various parameter groupings; however, those that are relevant to the results shown in Figure 4 are

$$
T_{B_{\max }} \sim \begin{cases}\frac{\alpha_{4} T_{T_{0}}^{S S}}{\alpha_{1} \mu_{1}+\alpha_{4} \mu_{4}}+\frac{1}{\left(\theta^{*}-\theta\right)} \frac{S_{1} \alpha_{1}\left(\theta^{*}\left(\delta_{H}+k_{1}\right)-\theta \delta_{H}\right)}{\left(\alpha_{1} \mu_{1}+\alpha_{4} \mu_{4}\right) k_{-1} \delta_{H}} & \theta<\theta^{*}, \\ \frac{1}{\varepsilon} \frac{S_{1}}{v \alpha_{4} \mu_{4}} & \theta^{*}<\theta<\theta_{L}, \\ \frac{1}{\varepsilon}\left(\frac{S_{1}}{v \alpha_{4} \mu_{4}}+\frac{S_{1}}{v \delta_{I_{T}} \mu_{4}}\left(1-\left(1-\frac{\delta_{I T} A(1,1)\left(\theta-\theta_{L}\right)}{\delta_{I_{T}} A(1,1)\left(\theta-\theta_{L}\right)+\Omega_{I}^{[15]} A\left(\theta_{L}-\theta^{*}, \theta_{L}\right)}\right)^{\frac{\delta_{T}}{\delta_{I T}}}\right)\right. & \theta_{L}<\theta,\end{cases}
$$

where $A(x, y)=\alpha_{2} \mu_{2} x+\alpha_{3} \mu_{3} y, \Omega_{I}^{[15]}$ is defined below equation (3.26), and

$$
\theta_{L}=\theta^{*}\left(\frac{\delta_{O}}{\alpha_{3}}+\frac{\alpha_{2} \mu_{2}}{\alpha_{2} \mu_{2}+\alpha_{3} \mu_{3}}\right)
$$

is a minor critical inhibitor concentration discussed below. We first note $T_{B_{\max }}$ is continuous at $\theta=\theta_{L}$, though curiously non-smooth, and that $T_{B_{\max }}=T_{B_{0}}^{[11]}$ (equation (3.21)) as $\theta \rightarrow \infty$, in agreement with the analysis of $\theta=1 / \varepsilon$. Secondly, we note that, if $\theta_{L}<\theta^{*}$, then only the first and third terms in (3.38) are relevant. For the low dose case (i.e. $\theta<\theta^{*}$ ), there is a constant contribution of blood tyrosine from the tissue phase and a $\theta$ dependant term from the liver, showing explicitly $T_{B}$ increases with increasing dose. Passing through the critical inhibitor dose, for an intermediate dose concentration of $\theta^{*}<\theta<\theta_{L}$, HPPD is rapidly eliminated and blood tyrosine equilibrates to $\varepsilon^{-1} S_{1} / v \alpha_{4} \mu_{4}=T_{B_{\infty}}^{[9]} / \varepsilon$ (see Section 3.1.4), at leading order; however, by $O(1)$ time the drug is exhausted so that the slower process of HPPD recovery occurs unhindered allowing no further build up of tyrosine (hence $T_{B_{\max }}$ is approximately constant in the intermediate range of $\theta$ ). For $\theta>\theta_{L}$, inhibitor concentrations in the liver remain high for long enough to maintain a low level of HPPD over an $O(1)$ time period, thereby allowing tyrosine to further build up in tissue beyond the $T_{B_{\infty}}^{[9]} / \varepsilon$ level, leading to a secondary rise in blood tyrosine concentration (as observed in Timescale 11, Section 3.1.5).

We can also deduce the minimum HPPD level from the analysis and find that

$$
H_{O_{\min }} \sim \begin{cases}1-\frac{\theta}{\theta^{*}} & \theta<\theta^{*}, \\ \varepsilon^{3} \frac{\delta_{O}+k_{-2}}{k_{2} I_{L}^{*}} & \theta^{*}<\theta,\end{cases}
$$


where $I_{L}^{*}=\left(\theta-\theta^{*}\right) I_{L}^{c}$, with constant $I_{L}^{c}=v \alpha_{2} \mu_{2}\left(\rho^{+}-\rho^{-}\right)^{-1} \max _{t>0}\left(e^{\rho^{+} t}-e^{\rho^{-} t}\right)$ (the constants $\rho^{ \pm}$ being defined in (3.101)). For $\theta<\theta^{*}$, only $\theta / \theta^{*}$ of inhibitor makes it into the liver and is insufficient to totally deplete HPPD. However, for $\theta>\theta^{*}$, HPPD concentration drops by several orders of magnitude and, as observed in Timescale 9 (Section 3.1.4), it is in a quasi-steady balance between production and inhibition. We note, that the minimum HPPD concentration is highly dependent on the reaction kinetics, unlike tyrosine, which is governed more by the mass transfer kinetics between phases.

The leading order estimates for $T_{B_{\max }}$ and $H_{O_{\min }}$ (dashed lines) are compared with the full solutions (solid lines) in Figure 4. Whilst, the predicted estimate of $T_{B_{\max }}$ is reasonable for $\theta<\theta^{*}$, they are fairly poor for $\theta>\theta^{*}$. The reason for this has already been highlighted in the discussion of Timescale 11 (Section 3.1.5) where the corrections terms are unduly large in comparison to $1 / \varepsilon$ with $\varepsilon=0.04$. Decreasing $\varepsilon$ improves agreement and the constant value in $\theta^{*}<\theta<\theta_{L}$ becomes more evident. However, with $\varepsilon=0.04$, the estimates for $H_{O_{\min }}$ are already very good (the dashed lines are more-or-less superimposed by the solid one).

\section{Data fitting}

The rat data used in the parameter fitting consists of time course measurements of the concentrations of tyrosine and the inhibitor nitisinone in the blood, i.e. $T_{B}$ and $I_{B}$ in the model (see Appendix B for animal experimentation details). In the experiments three different doses were investigated, namely at $0.5,1$ and $2 \mathrm{mg} / \mathrm{kg}$; in each case, three rats (nine in total) were given the same dose and there were three untreated rats used as control. The measurements were taken over seven days, with seven data points taken on day $1(t=0,0.5,1,2,4,8,12$ hours), two on day $2(t=24,36)$ and then once daily afterwards $(t=48,72,96,120,144,168)$. A key feature of the experimental results is that the peak blood tyrosine concentration is roughly the same across the three doses, which motivated the consideration of the large dose case $I_{B}(0)=O(1 / \varepsilon)$ in the analysis of Section 3.1.

It is possible to attempt parameter estimation by fitting the full model solutions to the available data using various software packages, and though they often provide detailed statistical analysis of the reliability of fit it is difficult to obtain insights into why some parameters are fitted more reliably than others. Here, we will fit the data to the simpler versions of the model, motivated by the analysis of 3.1, which helps to identify which parameters the data is able to reliably inform. The "fit" command in the gnuplot package was used to fit to the inhibitor data and for the tyrosine data the "FME" package was implemented within the programming language $\mathrm{R}$; in both cases, the Levenberg-Marquardt minimisation algorithm was used.

The asymptotic analysis of Section 3.1 showed that following an initial transient the blood inhibitor profile in dimensional terms settles to

$$
I_{B} \sim \frac{I_{0} V_{B}}{\mu_{3} V_{T}} e^{-\delta_{I T} t},
$$

in a $t=O(1)$ timescale (see Section 3.1.5). The predicted exponential decay agrees with the data following a short time period after bolus ingestion. Equation (4.1) has one parameter group $\left(\theta_{0}=\right.$ $\left.I_{0} V_{B} / \mu_{3} V_{T}\right)$ and one free parameter $\left(\delta_{I_{T}}\right)$, which were fitted to raw data and to a logged version of the raw data, from $t=0.2-4$ days (chopping off the initial transient and longer time points at which the measurements are at the same level as noise). Figure 6 shows that the simple exponential decay model fits very well the experimental data and the fitting procedure determined robust estimates for the values of $\theta_{0}$ and $\delta_{I_{T}}$. The estimates are shown in Table 4 and across all the initial doses we find that $\delta_{I_{T}} \approx 1.5-1.75 \mathrm{day}^{-1}$ and the ratio between the initial doses are reasonably consistent with the ratios 
of $I_{0}$ in $\theta_{0}$. Expression (4.1) suggests that the clearance of available inhibitor occurs predominantly in tissues other than the liver, and not through binding with HPPD in the liver.

For fitting with the tyrosine data we simplified the composite solution (3.34) by removing the fast timescale terms to a form that describes changes on an $O(1)$ day timescale (consistent with the data), namely

$$
T_{B}^{e}=Q_{1}+Q_{2}\left(H(T-t)\left(\left(1-e^{-Q_{3} t}\right)+H(t-T) Q_{4} e^{-\left(Q_{3}+Q_{5}\right)(t-T)}\right),\right.
$$

where

$$
T=\frac{\ln (\lambda)+Q_{6}}{Q_{7}}
$$

Here, $\lambda=I_{0} / \bar{I}_{0}$, where $I_{0}$ and $\bar{I}_{0}$ are the initial available inhibitor concentrations, with the latter corresponding to the blood concentration following a $1 \mathrm{mg} / \mathrm{kg}$ dose; this is the only data fitting parameter that consists of the initial dose. The seven $Q_{i}$ constants are parameters to be fitted to the data and are defined as follows in terms of the dimensional model parameters,

$$
\begin{aligned}
& Q_{1}=\frac{V_{T}\left(\delta_{T_{T}} T_{1} \alpha_{1}\left(B_{0} \delta_{H}+V_{L} k_{1} \delta_{O}\right)\left(V_{B} \alpha_{4}+V_{T}^{2} \delta_{T_{T}}\right)+V_{T} V_{L} T_{0} B_{0} A^{S S} \delta_{H} \delta_{T_{T}} k_{-1} \alpha_{4}\right)}{V_{L} T_{0} B_{0} A^{S S} \delta_{H} k_{-1}\left(V_{B} \alpha_{1} \mu_{1} \alpha_{4}+V_{T}^{2} \delta_{T_{T}}\left(\alpha_{1} \mu_{1}+\alpha_{4} \mu_{4}\right)\right)}, \\
& Q_{2}=\frac{T_{1}\left(V_{B} \alpha_{4}+V_{T}^{2} \delta_{T_{T}}\right)}{V_{B} T_{0} \alpha_{4} \mu_{4}}, \\
& Q_{3}=\delta_{T_{T}}, \\
& Q_{4}=\psi^{[14]}=\frac{V_{B} \alpha_{4}^{2} \mu_{4}}{\left(V_{B} \alpha_{4}+V_{T}^{2} \delta_{T_{T}}\right)\left(\alpha_{1} \mu_{1}+\alpha_{4} \mu_{4}\right)}, \\
& Q_{5}=\Omega_{T}^{[15]}-\delta_{T_{T}}=\frac{\alpha_{1} \mu_{1} \alpha_{4}}{V_{T}\left(\alpha_{1} \mu_{1}+\alpha_{4} \mu_{4}\right)}, \\
& Q_{6}=\frac{1}{\delta_{I_{T}}} \ln \left(\frac{\bar{I}_{0} V_{B}^{2} \alpha_{2} \mu_{2} \alpha_{3} \delta_{I_{T}}}{B_{0}\left(V_{B} \alpha_{2} \mu_{2} \alpha_{3}+V_{T}^{2} \delta_{I_{T}}\left(\alpha_{2} \mu_{2}+\alpha_{3} \mu_{3}\right)\right)}\right) \\
& Q_{7}=\delta_{I_{T}} .
\end{aligned}
$$

The constants $Q_{1}$ and $Q_{2}$ are the dimensional form of $T_{B_{0}}^{S S}$ (leading order pretreated blood tyrosine level) and $T_{B_{0}}^{[11]}$ (leading order maximum blood tyrosine level), respectively. The value $t=T$ corresponds to $t=\eta_{12}(\varepsilon)+\eta_{13}(0)$ in the analysis, where the dose dependent term $\lambda$ has been separated from the rest of the constants in $Q_{6}$. The same seven parameters were simultaneously fitted to the data for the three doses, which are distinguished in the fitting process by setting the values of $\lambda$ to be $0.5,1$ and 2 to represent the $0.5,1,2 \mathrm{mg} / \mathrm{kg}$ doses, respectively. A set of fitted values are shown in Table 4(b) and Figure 7 shows that the solution curve matches reasonably well with data. As is usual when parameter fitting, there exists numerous local minima and it was found that $Q_{1}, Q_{2}$ and $Q_{3}$ were generally insensitive to initial data, whilst there was sensitivity in the other parameters. Nevertheless, a pleasing result is that across a broad range of initial estimates, the values for $Q_{7}=\delta_{I_{T}}$ was found to be consistent with those in Table 4(a) determined from independent data. 
(a)

\begin{tabular}{|c|c|c|c|c|c|c|c|}
\hline \multirow{2}{*}{ parameter } & dose & \multicolumn{2}{|c|}{$0.5 \mathrm{mg} / \mathrm{kg}$} & \multicolumn{2}{c|}{$1 \mathrm{mg} / \mathrm{kg}$} & \multicolumn{2}{|c|}{$2 \mathrm{mg} / \mathrm{kg}$} \\
\cline { 2 - 8 } & units & raw & logged & raw & logged & raw & logged \\
\hline$\delta_{I_{T}}$ & day $^{-1}$ & 1.56 & 1.50 & 1.72 & 1.74 & 1.50 & 1.74 \\
$\theta_{0}$ & $\mu$ mole $/ \mathrm{ml}$ & 1.81 & 1.68 & 4.46 & 4.45 & 6.80 & 7.69 \\
\hline
\end{tabular}

(b)

\begin{tabular}{|c|c|c|c|c|c|c|c|}
\hline $\begin{array}{c}\text { fitted parameter } \\
\text { units }\end{array}$ & $\begin{array}{c}Q_{1} \\
\mu \mathrm{mole} / \mathrm{ml}\end{array}$ & $\begin{array}{c}Q_{2} \\
\mu \mathrm{mole} / \mathrm{ml}\end{array}$ & $\begin{array}{c}Q_{3} \\
\mathrm{day}^{-1}\end{array}$ & $\begin{array}{c}Q_{4} \\
\text { none }\end{array}$ & $\begin{array}{c}Q_{5} \\
\mathrm{day}^{-1}\end{array}$ & $\begin{array}{c}Q_{6} \\
\mathrm{day}^{-1}\end{array}$ & $\begin{array}{c}Q_{7} \\
\mathrm{day}^{-1}\end{array}$ \\
\hline value & 0.097 & 2.15 & 1.14 & 0.306 & 1.41 & 2.42 & 1.66 \\
\hline
\end{tabular}

Table 4. Tables showing a set of estimated values from the parameter fitting procedures to data. Table (a) shows estimates of $\delta_{I_{T}}$ and $\theta_{0}=I_{0} V_{B} / \mu_{3} V_{T}$ from fitting equation (4.1) to blood inhibitor concentration data between 0.2 and 4 days; all values are robust to different initial estimates. Table (b) shows estimates of the $Q_{i}$ s from fitting (4.2) to measured blood tyrosine data across 3 different doses; see text for discussion.
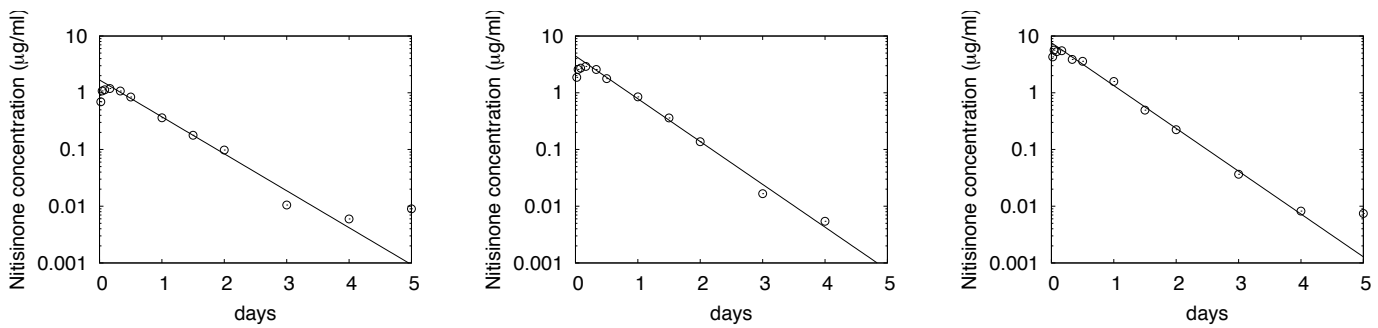

FIG. 6. Parameter fitted solutions of blood inhibitor concentration to data for the $0.5 \mathrm{mg} / \mathrm{kg}$ (left), $1 \mathrm{mg} / \mathrm{kg}$ (middle) and $2 \mathrm{mg} / \mathrm{kg}$ (right) cases. The fitted points are from $t=0.2$ days (i.e. not accounting for the initial phase of inhibitor absorption from the digestive tract) to $t=4$ days (neglecting the outlier points that appear from $t>4$ which are presumably at the noise level of the sampling).

Contained within these $Q_{i}$ 's are many parameters and this analysis suggests that it is not possible to discern all of these given the data, the best we can do is get approximate values for the relationships indicated. The only parameters that it does help to pin down are $\delta_{I_{T}}$ and $\delta_{T_{T}}$, which highlights the important role of the tissue volume in controlling the dynamics of tyrosine and inhibitor concentrations in the rat. A further issue highlighted by the fitting procedure is the unfortunate absence of key data points between days 2 and 3 . We observe that the model predicts that the effect of increasing the dose is to delay the collapse of tyrosine concentration to pretreated values and more data values would help to (1) provide more data points to establish more robust values for parameters $Q_{4}, Q_{5}, Q_{6}$ and $Q_{7}$ and (2) verify whether or not this dose-delay relationship is true. It is worth noting from (4.3), that $\delta_{I_{T}}=Q_{7}$ governs the length of the delay difference between doses.

\section{Discussion}

In this paper we have developed a mechanistic systems pharmacology model that covers the kinetics and dynamics of the exposure of nitisinone in rats and its effects on tyrosine level in the liver, blood and a generic tissue compartment. The use of asymptotic analysis has allowed us to excise the process into a 

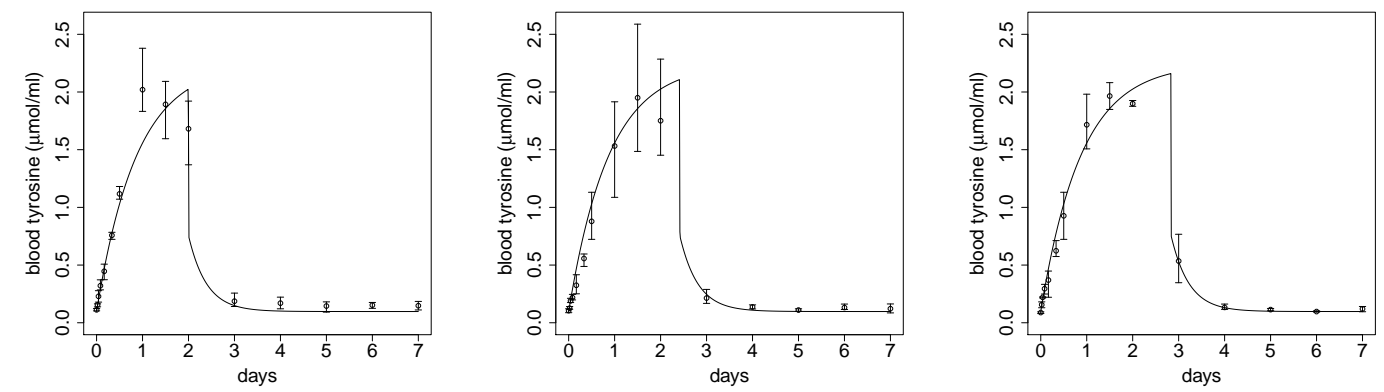

FIG. 7. Parameter fitted solutions of blood tyrosine concentration to data for $0.5 \mathrm{mg} / \mathrm{kg}$ (left), $1 \mathrm{mg} / \mathrm{kg}$ (middle) and $2 \mathrm{mg} / \mathrm{kg}$ (right). Each data point, indicated by the circles, is the mean of three data points, the error bars showing the minimum and maximum data values. The solid line shows the graph of equation (4.2) using the fitted data listed in Table 4(b).

number of steps and isolate their corresponding dominant events. As a practical application, the results of the time-scale analysis has helped us to determine which parameter combinations are identifiable from an animal experiment that involves a single oral dose of nitisione at three doses and in which tyrosine and inhibitor concentrations are measured only in the blood. In this scenario, we have learned that most of the model parameters are not identifiable from these time courses. This situation could be improved if tyrosine and inhibitor concentrations are measured in a representative tissue; however this would require terminal animal sampling. Alternatively, parameter values may be either directly measured (in vitro or in vivo) or predicted through the use of quantitative structure-activity relationship (QSAR) models. Finally, we achieved a good fitting of the model to the available data at all three doses nitisinone administered, and we were able to estimate the value of the identifiable parameter combinations for those cases.

Of particular relevance are two formulae from the asymptotic analysis:

- Formula (3.35) provides an explicit prediction of the critical concentration of the inhibitor at which tyrosine concentration starts to build up. For a particular HPPD inhibitor, this critical concentration is governed by the transport and partitioning of the inhibitor across the liver/blood/tissue phases and not by the specific values of the HPPD reaction parameters (this assumes a fast-acting inhibitor). Since it is usually possible to predict these parameters using simple QSAR approaches, this result is potentially very useful for finding appropriate dosing regimes.

- Formula (4.1) provides a simple description of the inhibitor decay in blood based on the partition coefficient between blood and tissue and the inhibitor decay in tissue. It emphasises that the critical missing measurement in many animal studies of HPPD inhibition is the decay rate of the inhibitor in tissue.

We note that asymptotic analysis is widely applied to dynamical systems, but seems to be rarely used for pharmacological models. This is likely due to the system sizes of these models being typically large, making computational approaches seemingly the only option. However, though the complexity of the analysis generally increases with system size, the underlying methodology is always the same and can, in principle, be applied to a system of any size. The mathematics package Maple was used to undertake some of the analysis in this paper (namely generating equations for each timescale, perform series expansions, solving differential and algebraic equations), but the technical details of matching and establishing timescale breakdown was done by hand; as yet there does not appear to be any software that 
automates this process. The application of asymptotic analysis has its challenges, but it can provide substantial rewards through drawing out fundamental characteristics of a model's dynamics (hence insights into the application), often in terms of relatively simple, understandable expressions that would be difficult or impossible to obtain using other tools (e.g. in obtaining the critical concentration $\theta^{*}$ in Section 3.2). Consequently, this analytical approach should be considered part of the standard "tool kit" used in the study of small to medium sized pharmacological models.

This model can be extended to support novel HPPD inhibitors development, either for agro-chemical or clinical use. The model can support both single or repeat dose exposure scenarios for any HPPD inhibitor, variable concentrations of tyrosine in blood and tissue due to different dietary patterns and also allows for differences in TAT activation across species. Ideally, the model should rely on QSAR predictions and/or in vitro measurements in order to minimise or eliminate animal experiments in early research. In order to estimate the viability of this goal, we need to classify the original model parameters into a number of groups:

- Values of the compartment volumes $\left(V_{T}, V_{B}\right.$ and $\left.V_{L}\right)$ are readily available in the literature for all three species of interest (see Brown et al. (1997)). External variable inputs like $T_{0}$ (tyrosine produced in tissue) and $T_{1}$ (tyrosine introduced in the liver from the gut) are dependent on the diet. There have been many studies in rats and humans in this area (see references below) from which to extract appropriate estimates, although we have the added complication that $T_{0}$ is tissue-specific. Fortunately, we have direct control over another variable input, the inhibitor dose. Finally, we have an unknown internal input which is the generation of HPPD in the liver, but we have measurements of HPPD in the liver in both rats and mice.

- Normal tyrosine concentration measurements in the blood are available for mice, rats and humans (Hall et al. (2001) and references below). For rats, tyrosine concentrations in the liver have also been measured; in this species in fact, the circadian behaviour of TAT concentration has received quite a bit of attention (Wurtman and Axelrod, 1967; Kato \& Saito, 1980; Reynolds et al., 1971; Ross et al., 1973; Cahill et al., 1981). In rats and mice, tyrosine concentration has been measured in certain compartments like ocular fluid; in addition, TAT activity levels are also available in the liver (Lock et al., 1996, 2000).

- Time courses in mice and rats available in the literature (Ellis et al., 1995, 1996; Lock et al., 1996, 2000) may be enough to parametrise both the tyrosine transformation into HPPA (see also Rosenberg \& Litwack (1970)), mediated by TAT, as well as the subsequent transformation of HPPA into homogentisic acid (mediated by homogentisic acid oxidase). Otherwise, appropriate in vitro measurements could be made; this would be especially relevant for humans. Normal concentrations of TAT and HPPA in the liver, tyrosine in blood and homogentisic acid, and various phenolics in urine has been measured for rats and humans (see references above).

- For a novel inhibitor, it is possible to use QSAR models to predict transfer rates between blood and liver and blood and tissue (parameters $\alpha_{2}$ and $\alpha_{3}$ respectively in our model), as well as partition coefficient and plasma binding fractions. On the other hand, the binding of the inhibitor to HPPD, can be measured in vitro to produce the appropriate values for $k_{2}$ and $k_{-2}$.

- As a result of the above considerations, the only parameters not available, at least theoretically, by either QSAR modelling or in vitro measurements are the degradation constants, represented by $\delta \mathrm{s}$ in our model. The ones corresponding to an inhibitor $\left(\delta_{I_{B}}, \delta_{I_{L}}, \delta_{I_{T}}\right)$; corresponding to blood, liver and tissue) may not play a big role for a strong binding inhibitor. The corresponding 
28 of 55

ones for tyrosine $\left(\delta_{T_{B}}, \delta_{T_{L}}, \delta_{T_{T}}\right)$, HPPA $\left(\delta_{P}\right)$ and homogentisic acid $\left(\delta_{R}\right)$ could be computed from the model presented in this paper, once the appropriate reaction constants $\left(k_{2}, k_{-2}\right.$ and $\left.\delta_{H}\right)$ are available. The existent of time course for concentrations of various inhibitors should provide enough data to identify most if not all of these parameters. In fact, a predicted value for $\delta_{T_{T}}$ (as well as for $\delta_{I_{T}}$ in the case of nitisinone) is already forthcoming from the computations carried out in this paper. In any case, once these values are established, they can be used for any inhibitor of interest without the need for re-computing them.

Consequently, it should be possible to run this model to predict tyrosine concentration in the various compartments considered after the administration of novel HPPD inhibitors across species. Being a mechanistic model, it does not require an array of such novel inhibitors for training purposes although the accuracy of the model will depend on the validity of the QSAR predictors or additional in vitro and in vivo inputs.

As well as a virtual test-bed for the development of new HPPD inhibitors, this model can be used to illuminate the possible source of the intriguing differences in toxicity between rats and other species. The results of the simulation seem to indicate that tyrosine measurements in the blood do not convey the full picture; for that we need to know the concentration of tyrosine in the tissue of interest as well as in the liver. It is possible that in rats, given the circadian nature of TAT activity, the effect of the inhibitor is to elevate the concentration of tyrosine in the liver to the point that tyrosine is transferred in great quantity into various tissues, causing there the observed toxicities. Perhaps, in mice and humans, HPPA does not convert back to tyrosine but instead either accumulate harmlessly in some form in the liver or it is simply excreted as phenolics in the urine. The model can be used to evaluate the plausibility of these hypothesis.

The model presented in this paper looks at the effects of HPPD inhibition by nitisinone in the tyrosine catabolism pathway; however the model lends itself to be readily adapted to other diseases involving this pathway. For example, alkaptonuria is a rare, painful and degenerative disease caused by a mutation of the homogentisic acid oxidase enzyme (see Figure 1). The lack of this enzyme causes a build-up of homogentisic acid which in turn produces the debilitating phenotype in this disease (Fernández-Cañon et al., 1996). Similar to type I tyrosinaemia, targeting HPPD with nitisinone shows some promise (Suwannarat et al., 2005). This mathematical model could provide the basis to help predict the potential effects of nitisinone dosing and the resultant reduction in urinary homogentisic acid over the time period of treatment. In addition, the model can support chronic exposure scenarios and the influences of ageing or sex differences that may introduce altered behaviour in dietary absorption of tyrosine into the liver (input parameter $T_{1}$ in the model) or in the production of the enzymes included in the model such as HPPD (through the input parameter $B_{0}$ ) and TAT.

Overall, the model has the scope to contribute to the reduction, replacement and refinement of animal experimentation in research and development.

Acknowledgements The authors wish to thank the National Centre for the Replacement, Refinement and Reduction of Animals in Research (NC3Rs) and the organisers of the 2011 Maths in Medicine Studygroup, University of Reading, for facilitating the initial interaction amongst the authors. A special thanks also to colleagues at Syngenta Ltd., Kim Travis, Paul Hinderliter, David Cowie, Mike Langford and Tim Hawkes for some of the nitisinone data and valued comments. 


\section{References}

Brown, R.P., Delp, M.D., Lindstedt, S.L., Rhomberg, L.R. \& Beliles, R.P. (1997). Physiological parameter values for physiologhically based pharmacokinetic models. Toxicol.Ind. Health, 13(4):407-84.

Cahill, Anne L., And Charles F. Ehret (1981). Circadian Variations in the Activity of Tyrosine Hydroxylase, Tyrosine Aminotransferase, and Tryptophan Hydroxylase: Relationship to Catecholamine Metabolism. Journal of Neurochemistry 37(5):1109-15. doi:10.1111/j.14714159.1981.tb04660.x.

Ellis M.K., Whitfield A.C., Gowans L.A., Auton T.R., Provan M.W., Lock E.A. \& SMith L.L. (1995) Inhibition of 4-Hydroxyphenylpyruvate Dioxygenase by 2-(2-Nitro4-trifluoromethylbenzoyl)-cyclohexane- 1,3-dione and 2-(2-Chloro-4-methanesulfonylbenzoyl)cyclohexane- 1, 3-dione. Tox App. Pharm. 133:12-19.

Ellis, M. K., Whitfield, A. C., Gowans, L. A., Auton, T. R., Provan, W. M., Lock, E. A., LEE , D. AND SMITH, L. L (1996). Characterization of the interaction of NTBC with rat hepatic HPPD. Chem. Res. Toxicol. 9:24-27.

Fernndez-Cañón, Jos M., Begoña Granadino, Daniel Beltrán-Valero De Bernabé, Mónica Renedo, Elena Fernández-Ruiz, Miguel A. Peñalva and Santiago Rodríguez De CóRdobA (1996). The Molecular Basis of Alkaptonuria. Nature Genetics 14(1):1924. doi:10.1038/ng0996-19.

Goldsmith L.A. (1983) Tyrosinaemia II: lessons in molecular pathology. Pediatr. Dermatol 1:25-34.

Hall M.G., Wilks M.F., Provan W.M., Eksborg S. \& Lumholtz B. (2001) Pharmacokinetics and pharmacodynamics of NTBC and mesotrione, inhibitors of HPPD following a single dose to healthy male volunteers. British Journal of Clinical Pharmacology 52:169-177

Henderson, J. Michael, Faraj, Bahjat A., Ali, Farouk M. and Rudman, Daniel (1981). Tyrosine Transaminase activity in normal and cirrhotic liver. Digestive Diseases and Sciences 26(2):124-128, 1981.

Holme E. \& LindstedT S. (1998). Tyrosinaemia type I and NTBC. J. Inherit. Metab. Dis. 21:507517.

Kato, Hideo, and Masayuki Saito (1980). Diurnal Variations in Response of Rat Liver Tyrosine Aminotransferase Activity to Food Intake. Biochimica et Biophysica Acta (BBA) - General Subjects 627(1):109-11. doi:http://dx.doi.org/10.1016/0304-4165(80)90128-2.

LEe, H.B. \& Blaufox, M.D. (1985). Blood Volume in the Rat. J. Nucl. Med. 26:72-76.

Lewis R.W. \& Botham J.W. (2013) A review of the mode of toxicity and relevance to humans of the triketone herbicide mesotrione. Crit. Rev. Toxicol. 43(3):185199

Lindblad, B., G. Lindstedt, S. Lindstedt, And M. Rundgren (1977). Purification and Some Properties of Human 4-Hydroxyphenylpyruvate Dioxygenase (I). Journal of Biological Chemistry 252(14):5073-84. 
Lock E.A., Gaskin P., Ellis M.K., Provan W.M., Robinson M., Smith L.L., PrisBYLLA M.P. \& MUTTER L.C. (1996) Tissue distribution of 2-(2-Nitro-4-trifluoromethylbenzoyl)cyclohexane- 1,3-dione (NTBC): effects on enzymes involved in tyrosine catabolism and relevance to ocular toxicity in the rat. Toxicol. Appl. Pharmacol. 141:439-447

Lock E.A., Ellis M.K., Gaskin P., Robinson M., Auton T.R., Provan W.M., Smith L.L., Prisbylla P., Mutter L.C. \& Lee D.L. (1998) From toxicological problem to therapeutic use: the discovery of the mode of action of NTBC, its toxicology and development as a drug. J. Inher. Metab. Dis. 21:498-506.

Lock, Edward A., Peter Gaskin, Martin K. Ellis, W. Mclean Provan, Mervyn RobinSON, AND LEWIS L. SMITH (2000). Tissue Distribution of 2-(2-Nitro-4-Trifluoromethylbenzoyl)Cyclohexane-1,3-Dione (NTBC) and Its Effect on Enzymes Involved in Tyrosine Catabolism in the Mouse. Toxicology 144(1-3):179-87. doi:10.1016/S0300-483X(99)00205-X.

Lock EA, Gaskin P, Ellis MK, Provan WM and Smith LL (2006). Tyrosinemia produced by 2-(2-nitro-4-trifluoromethylbenzoyl)-cyclohexane-1,3-dione (NTBC) in experimental animals and its relationship to corneal injury. Toxicology and Applied Pharmacology 215(1):9-16.

Lindstedt, S, E Holme, E. A Lock, O HJalmarson, and B Strandvik. Treatment of Hereditary Tyrosinaemia Type I by Inhibition of 4-Hydroxyphenylpyruvate Dioxygenase. The Lancet, Originally published as Volume 2, Issue 8823, 340, no. 8823 (October 3, 1992): 813-17. doi:10.1016/01406736(92)92685-9.

MCKIERnAN PJ (2006). Nitisinone in the Treatment of Hereditary Tyrosinaemia Type. Drugs 166(6):743-750.

Mitchell GA, Grompe M, LAmbert M (2001). Hypertyrosinaemia In: Scriver CR Beaudet AR, Sly W, editors. The metabolic and molecular basis of inherited disease. New York: McGraw-Hill 1777-1805.

Prisbylla MP, Onisko BC, Shribbs JM, Adams DO, LiU Y, Ellis MK, HaWkes TR, AND MUTTER LC (1993). The novel mechanism of action of the herbicidal triketones. Weeds 3:731-738.

Reynolds, Robert D., Van R. Potter, and Henry C. Pitot (1971). Response of Several Hepatic Adaptive Enzymes to a Shift from Low to High Protein Diet in Intact and Adrenalectomized Rats. The Journal of Nutrition 101(6):797-802.

Rosenberg, Joseph S., And Gerald Litwack (1970). Liver Cytosol Tyrosine Aminotransferase. Journal of Biological Chemistry 245(21):5677-84.

Ross, Douglas S., John D. Fernstrom, and Richard J. Wurtman (1973). The Role of Dietary Protein in Generating Daily Rhythms in Rat Liver Tryptophan Pyrrolase and Tyrosine Transaminase. Metabolism 22(9):1175-84. doi:10.1016/0026-0495(73)90205-9.

Sammartino A, Cerbella R, Cecio A, Crecchio G De, Federico A, Fronterre A (1987). The effects of diet on the ophthalmological, clinical and biochemical aspects of Richner-Hanhart syndrome: a morphological ultrastructural study of the cornea and conjunctiva. Int. Ophthamol. 10:203212 . 
Schulz A, ORT O, Beyer P, Kleinig H (1993) SC-0051, a 2-benzoyl-cyclohexane-1,3-dione bleaching herbicide, is a potent inhibitor of the enzyme p-hydroxyphenylpyruvate dioxygenase. FEBS Letts 318:162-166.

Suwannarat, Pim, Kevin Obrien, Monique B. Perry, Nancy Sebring, Isa Bernardini, Muriel I. KAiser-Kupfer, Benjamin I. Rubin, EKATERINA TSILOU, LynN H. GERBER, AND William A. GAHL (2005). Use of Nitisinone in Patients with Alkaptonuria. Metabolism 54(6):71928. doi:10.1016/j.metabol.2004.12.017.

Wurtman, R J, And J Axelrod (1967). Daily Rhythmic Changes in Tyrosine Transaminase Activity of the Rat Liver. Proceedings of the National Academy of Sciences of the United States of America 57(6):1594-98. 


\section{A. Asymptotic analysis}

Here we give details of the asymptotic analysis that we summarised in the Section 3 . The rescalings, equations, solutions and reasons for solution breakdown are presented for each timescale. In most cases the matching is straightforward and details of this are generally omitted for brevity. As in Section 3, the relevant variable rescalings for each timescale are presented first using the $\mapsto$ notation, which on substitution into (2.8a)-(2.8j) yields the corresponding system of equations. Against formality, when writing the timescales in the subsection headings, we treated the logarithmically large $(O(\ln (1 / \varepsilon)))$ terms the same as $O(1)$ quantities, since they are not that large for biologically relevant values of $\varepsilon$; however, in the analysis $1 \ll \ln (1 / \varepsilon)$ is formally applied. For each timescale, a very rough indication of the "real-world" time frame is given; these are approximations based on $\varepsilon=0.04$ and $t=1 \approx 16$ hours and all other parameters set to unity. Finally, it should be noted that most of the tedious algebra was undertaken using the mathematics package Maple (version 17).

Timescale 1: $t=O\left(\varepsilon^{3}\right)$

Corresponds to $t=O(4) \mathrm{s}$. We introduce the scalings

$$
\begin{aligned}
& T_{T} \mapsto T_{T}, \quad T_{B} \mapsto T_{B}, \quad T_{L} \mapsto T_{L}, \quad H_{P} \mapsto H_{P}, \quad H_{O} \mapsto H_{O}, \\
& R \mapsto R, \quad C \mapsto \varepsilon C, \quad I_{T} \mapsto \varepsilon^{2} I_{T}, \quad I_{B} \mapsto \frac{I_{B}}{\varepsilon}, \quad I_{L} \mapsto \varepsilon I_{L},
\end{aligned}
$$

and apply these to the nondimensional system (2.8) resulting in the equations

$$
\begin{aligned}
\frac{d T_{T}}{d t} & =\varepsilon^{3}\left(\delta_{T_{T}}\left(1-T_{T}\right)-\alpha_{4}\left(T_{T}-\mu_{4} T_{B}\right)\right) \\
\frac{d T_{B}}{d t} & =\varepsilon^{2}\left(\alpha_{4}\left(T_{T}-\mu_{4} T_{B}\right)+\alpha_{1}\left(T_{L}-\mu_{1} T_{B}\right)\right)-\varepsilon^{3} \delta_{T_{B}} T_{B} \\
\frac{d T_{L}}{d t} & =\varepsilon\left(S_{1}+k_{1} H_{P}-k_{-1} T_{L}\right)-\varepsilon^{2} v \alpha_{1}\left(T_{L}-\mu_{1} T_{B}\right)-\varepsilon^{3} \delta_{T_{L}} T_{L} \\
\frac{d H_{P}}{d t} & =\varepsilon\left(k_{-1} T_{L}-k_{1} H_{P}-\delta_{H} H_{O} H_{P}\right)-\varepsilon^{3} \delta_{P} H_{P} \\
\frac{d H_{O}}{d t} & =-\varepsilon k_{2} H_{O} I_{L}+\varepsilon^{3} \delta_{O}\left(1-H_{O}\right)+\varepsilon^{4} k_{-2} C \\
\frac{d R}{d t} & =\varepsilon\left(\delta_{H} H_{O} H_{P}-\delta_{R} R\right), \\
\frac{d C}{d t} & =k_{2} H_{O} I_{L}-\varepsilon^{3}\left(\delta_{C}+k_{-2}\right) C \\
\frac{d I_{T}}{d t} & =\alpha_{3} \mu_{3} I_{B}-\varepsilon^{3}\left(\alpha_{3}+\delta_{I_{T}}\right) I_{T} \\
\frac{d I_{B}}{d t} & =-\varepsilon^{2}\left(\alpha_{2} \mu_{2}+\alpha_{3} \mu_{3}\right) I_{B}-\varepsilon^{3} I_{B}+\varepsilon^{4} \alpha_{2} I_{L}+\varepsilon^{5} \alpha_{3} I_{T} \\
\frac{d I_{L}}{d t} & =v \alpha_{2} \mu_{2} I_{B}-k_{2} H_{O} I_{L}-\varepsilon^{2} v \alpha_{2} I_{L}+\varepsilon^{3}\left(k_{-2} C-\delta_{I_{L}} I_{L}\right)
\end{aligned}
$$

At leading order we have

$$
\begin{gathered}
T_{T_{0}}=T_{T_{0}}^{S S}, \quad T_{B_{0}}=T_{B_{0}}^{S S}, \quad T_{L_{0}}=T_{L_{0}}^{S S}, \quad H_{P_{0}}=H_{P_{0}}^{S S}, \\
H_{O_{0}}=1, \quad R_{0}=R_{0}^{S S}, \quad I_{B_{0}}=\Theta .
\end{gathered}
$$


For the remaining variables we note that $d\left(C_{0}+I_{L_{0}}\right) / d t=\alpha_{2} \mu_{2} v \Theta$, and hence

$$
\begin{gathered}
I_{T_{0}}=\alpha_{3} \mu_{3} \Theta t, \quad I_{L_{0}}=\frac{v \alpha_{2} \mu_{2} \Theta}{k_{2}}\left(1-e^{-k_{2} t}\right), \\
C_{0}=v \alpha_{2} \mu_{2} \Theta t-\frac{v \alpha_{2} \mu_{2} \Theta}{k_{2}}\left(1-e^{-k_{2} t}\right) .
\end{gathered}
$$

The linear accumulation of $I_{T_{0}}$ and $C_{0}$ leads to a change in balance at $t=O(1 / \varepsilon)$.

Timescale 2: $t=O\left(\varepsilon^{2}\right)$

Corresponds to $t=O(90)$ s. We rescale time such that $t \mapsto \varepsilon^{2} t$ and obtain the scalings

$$
\begin{aligned}
& T_{T} \mapsto T_{T}, \quad T_{B} \mapsto T_{B}, \quad T_{L} \mapsto T_{L}, \quad H_{P} \mapsto H_{P}, \quad H_{O} \mapsto H_{O}, \\
& R \mapsto R, \quad C \mapsto C, \quad I_{T} \mapsto \varepsilon I_{T}, \quad I_{B} \mapsto \frac{I_{B}}{\varepsilon}, \quad I_{L} \mapsto \varepsilon I_{L},
\end{aligned}
$$

applying these to the equations (2.8) results with

$$
\begin{aligned}
\frac{d T_{T}}{d t} & =\varepsilon^{2}\left(\delta_{T_{T}}\left(1-T_{T}\right)-\alpha_{4}\left(T_{T}-\mu_{4} T_{B}\right)\right) \\
\frac{d T_{B}}{d t} & =\varepsilon\left(\alpha_{4}\left(T_{T}-\mu_{4} T_{B}\right)+\alpha_{1}\left(T_{L}-\mu_{1} T_{B}\right)\right)-\varepsilon^{2} \delta_{T_{B}} T_{B}, \\
\frac{d T_{L}}{d t} & =S_{1}+k_{1} H_{P}-k_{-1} T_{L}-\varepsilon v \alpha_{1}\left(T_{L}-\mu_{1} T_{B}\right)-\varepsilon^{2} \delta_{T_{L}} T_{L}, \\
\frac{d H_{P}}{d t} & =k_{-1} T_{L}-k_{1} H_{P}-\delta_{H} H_{O} H_{P}-\varepsilon^{2} \delta_{P} H_{P}, \\
\frac{d H_{O}}{d t} & =-k_{2} H_{O} I_{L}+\varepsilon^{2}\left(\delta_{O}\left(1-H_{O}\right)+k_{-2} C\right) \\
\frac{d R}{d t} & =\delta_{H} H_{O} H_{P}-\delta_{R} R \\
\frac{d C}{d t} & =k_{2} H_{O} I_{L}-\varepsilon^{3}\left(\delta_{C}+k_{-2}\right) C \\
\frac{d I_{T}}{d t} & =\alpha_{3} \mu_{3} I_{B}-\varepsilon^{2}\left(\alpha_{3}+\delta_{I_{T}}\right) I_{T} \\
\frac{d I_{B}}{d t} & =-\varepsilon\left(\alpha_{2} \mu_{2}+\alpha_{3} \mu_{3}\right) I_{B}-\varepsilon^{2} I_{B}+\varepsilon^{3}\left(\alpha_{2} I_{L}+\alpha_{3} I_{T}\right), \\
\varepsilon \frac{d I_{L}}{d t} & =v \alpha_{2} \mu_{2} I_{B}-k_{2} H_{O} I_{L}-\varepsilon^{2}\left(v \alpha_{2} I_{L}-k_{-2} C\right)-\varepsilon^{3} \delta_{I_{L}} I_{L} .
\end{aligned}
$$

At leading order we obtain the explicit leading order solutions

$$
\begin{gathered}
T_{T_{0}}=T_{T_{0}}^{S S}, \quad T_{B_{0}}=T_{B_{0}}^{S S}, \quad I_{B_{0}}=\Theta, \quad C_{0}=v \alpha_{2} \mu_{2} \Theta t \\
H_{O_{0}}=1-v \alpha_{2} \mu_{2} \Theta t . \quad I_{T_{0}}=\alpha_{3} \mu_{3} \Theta t, \quad I_{L_{0}}=\frac{v \alpha_{2} \mu_{2} \Theta}{k_{2}\left(1-\Theta v \alpha_{2} \mu_{2} t\right)},
\end{gathered}
$$


two coupled ordinary differential equations

$$
\begin{aligned}
& \frac{d T_{L_{0}}}{d t}=S_{1}+k_{1} H_{P_{0}}-k_{-1} T_{L_{0}}, \\
& \frac{d H_{P_{0}}}{d t}=-k_{1} H_{P_{0}}+k_{-1} T_{L_{0}}-\delta_{H}\left(1-v \alpha_{2} \mu_{2} \Theta t\right) H_{P_{0}},
\end{aligned}
$$

and the decoupled

$$
\frac{d R_{0}}{d t}=\delta_{H}\left(1-v \alpha_{2} \mu_{2} \Theta t\right) H_{P_{0}}-\delta_{R} R_{0}
$$

Here, $H_{O_{0}} \rightarrow 0$ and $I_{L_{0}} \rightarrow \infty$ as $t \rightarrow \eta_{3}(\varepsilon)^{-}<\infty$, where $\eta_{3}(0)=1 / v \alpha_{2} \mu_{2} \Theta$, leading to a new timescale.

Timescale 3: $t=\varepsilon^{2} \eta_{3}(\varepsilon)+O\left(\varepsilon^{5 / 2}\right)$

Corresponds to $t \approx 90 \mathrm{~s}$. Here, $\eta_{3}(\varepsilon)$ is defined such that $\eta_{3}(0)=1 / v \alpha_{2} \mu_{2} \Theta$, and we obtain the scalings: $t \mapsto \varepsilon^{2} \eta_{3}(\varepsilon)+\varepsilon^{5 / 2} t$,

$$
\begin{aligned}
& T_{T} \mapsto T_{T}, \quad T_{B} \mapsto T_{B}, \quad T_{L} \mapsto T_{L}, \quad H_{P} \mapsto H_{P}, \quad H_{O} \mapsto \varepsilon^{1 / 2} H_{O}, \\
& R \mapsto R, \quad C \mapsto C, \quad I_{T} \mapsto \varepsilon I_{T}, \quad I_{B} \mapsto \frac{I_{B}}{\varepsilon}, \quad I_{L} \mapsto \varepsilon^{1 / 2} I_{L},
\end{aligned}
$$

and apply these to the system (2.8) resulting in

$$
\begin{aligned}
\frac{d T_{T}}{d t} & =\varepsilon^{5 / 2}\left(\delta_{T_{T}}\left(1-T_{T}\right)-\alpha_{4}\left(T_{T}-\mu_{4} T_{B}\right)\right) \\
\frac{d T_{B}}{d t} & =\varepsilon^{3 / 2}\left(\alpha_{4}\left(T_{T}-\mu_{4} T_{B}\right)+\alpha_{1}\left(T_{L}-\mu_{1} T_{B}\right)\right)-\varepsilon^{5 / 2} \delta_{T_{B}} T_{B} \\
\frac{d T_{L}}{d t} & =\varepsilon^{1 / 2}\left(S_{1}+k_{1} H_{P}-k_{-1} T_{L}\right)-\varepsilon^{3 / 2} v \alpha_{1}\left(T_{L}-\mu_{1} T_{B}\right)-\varepsilon^{5 / 2} \delta_{T_{L}} T_{L} \\
\frac{d H_{P}}{d t} & =\varepsilon^{1 / 2}\left(k_{-1} T_{L}-k_{1} H_{P}\right)-\varepsilon \delta_{H} H_{O} H_{P}-\varepsilon^{5 / 2} \delta_{P} H_{P} \\
\frac{d H_{O}}{d t} & =-k_{2} H_{O} I_{L}+\varepsilon^{2}\left(\delta_{O}+k_{-2} C\right)-\varepsilon^{5 / 2} \delta_{O} H_{O} \\
\frac{d R}{d t} & =-\varepsilon^{1 / 2} \delta_{R} R+\varepsilon \delta_{H} H_{O} H_{P}, \\
\frac{d C}{d t} & =\varepsilon^{1 / 2} k_{2} H_{O} I_{L}-\varepsilon^{5 / 2}\left(\delta_{C}+k_{-2}\right) C \\
\frac{d I_{T}}{d t} & =\varepsilon^{1 / 2} \alpha_{3} \mu_{3} I_{B}-\varepsilon^{5 / 2}\left(\alpha_{3}+\delta_{I_{T}}\right) I_{T} \\
\frac{d I_{B}}{d t} & =-\varepsilon^{3 / 2}\left(\alpha_{2} \mu_{2}+\alpha_{3} \mu_{3}\right) I_{B}-\varepsilon^{5 / 2} I_{B}+\varepsilon^{3} \alpha_{2} I_{L}+\varepsilon^{7 / 2} \alpha_{3} I_{T} \\
\frac{d I_{L}}{d t} & =v \alpha_{2} \mu_{2} I_{B}-k_{2} H_{O} I_{L}-\varepsilon^{3 / 2} \alpha_{2} v I_{L}+\varepsilon^{2} k_{-2} C-\varepsilon^{5 / 2} \delta_{I_{L}} I_{L} .
\end{aligned}
$$


All variables except HPPD, $H_{O_{0}}$, and liver inhibitor concentration, $I_{L_{0}}$ are, at leading order, locked at the $t=\eta_{3}$ values on the previous timescale. namely

$$
\begin{gathered}
C_{0}=1, \quad T_{T_{0}}=T_{T_{0}}^{S S}, \quad T_{B_{0}}=T_{B_{0}}^{S S}, \quad I_{B_{0}}=\Theta, \quad I_{T_{0}}=\frac{\alpha_{3} \mu_{3}}{\alpha_{2} \mu_{2} v}, \\
T_{L_{0}}=T_{L_{0}}^{[2]}\left(\eta_{3}\right), \quad H_{P_{0}}=H_{P_{0}}^{[2]}\left(\eta_{3}\right), \quad R_{0}=R_{0}^{[2]}\left(\eta_{3}\right),
\end{gathered}
$$

where the superscript "[2]" represents the Timescale 2 solution.

Between the ODEs for $H_{O_{0}}$ and $I_{L_{0}}$ we deduce

$$
I_{L_{0}}=v \alpha_{2} \mu_{2} \Theta t+H_{O_{0}}
$$

leading to a Riccati equation

$$
\frac{d H_{O_{0}}}{d t}=-k_{2} H_{O_{0}}\left(v \alpha_{2} \mu_{2} \Theta t+H_{O_{0}}\right)
$$

which solves to give

$$
H_{O_{0}}=\frac{\sqrt{2 v \alpha_{2} \mu_{2} \Theta} e^{-k_{2} \Theta v \alpha_{2} \mu_{2} t^{2} / 2}}{\sqrt{\pi k_{2}}\left(1+\operatorname{erf}\left(\sqrt{\frac{k_{2} \Theta v \alpha_{2} \mu_{2}}{2}} t\right)\right)},
$$

where $\operatorname{erf}(x)=\frac{2}{\sqrt{\pi}} \int_{0}^{x} e^{-z^{2}} d z$; this expression for $H_{O_{0}}$ can be shown to be universally valid to leading order for the first three timescales as $\varepsilon \rightarrow 0$. Of particular note

$$
H_{O_{0}} \sim \frac{\sqrt{v \alpha_{2} \mu_{2} \Theta} e^{-k_{2} \Theta v \alpha_{2} \mu_{2} t^{2} / 2}}{\sqrt{2 \pi k_{2}}} \text { as } t \rightarrow \infty .
$$

On this timescale the HPPD concentration descends further with a balance shift when $I_{L_{0}} H_{O_{0}}=O\left(\varepsilon^{2}\right)$ and the reversible reaction between HPPD, inhibitor and complex begins to equilibrate. The balance $I_{L_{0}} H_{O_{0}}=O\left(\varepsilon^{2}\right)$ occurs when

$$
\frac{\left(v \alpha_{2} \mu_{2} \Theta\right)^{3 / 2}}{\sqrt{2 \pi k_{2}}} t e^{-k_{2} \Theta v \alpha_{2} \mu_{2} t^{2} / 2} \sim \varepsilon^{2}
$$

which on taking logs of both sides and ordering terms for large $t$, we obtain

$$
\frac{k_{2} v \alpha_{2} \mu_{2} \Theta}{2} t^{2}-\ln (t)-\ln \left(\frac{\left(v \alpha_{2} \mu_{2} \Theta\right)^{3 / 2}}{\sqrt{2 \pi k_{2}}}\right)=2 \ln (1 / \varepsilon)
$$

whereupon we write $t=t_{0}+t_{1}+t_{2}+t_{3}+\ldots$, so that $t_{i} \ll t_{i-1}$, and solve by balancing terms until we get to a $t_{i}$ that effects an $O(1)$ change; here $t_{0}$ and $t_{1}$ form the components of $\eta_{4}(\varepsilon)$ below and $t_{2}$ balances with the $O(1)$ terms in (A.23). 
Timescale 4: $t=\varepsilon^{2} \eta_{3}(\varepsilon)+\varepsilon^{5 / 2} \eta_{4}(\varepsilon)+O\left(\varepsilon^{5 / 2} / \ln (1 / \varepsilon)^{1 / 2}\right)$

Corresponds to $t \approx 120 \mathrm{~s}$. Here,

$$
\eta_{4}(\varepsilon)=\frac{2 \ln (1 / \varepsilon)^{1 / 2}}{\sqrt{k_{2} v \alpha_{2} \mu_{2} \Theta}}+\eta_{41}(\varepsilon)=\frac{2 \ln (1 / \varepsilon)^{1 / 2}}{\sqrt{k_{2} v \alpha_{2} \mu_{2} \Theta}}+\frac{\ln (\ln (1 / \varepsilon))}{4 \sqrt{k_{2} v \alpha_{2} \mu_{2} \Theta} \ln (1 / \varepsilon)^{1 / 2}}
$$

where $\eta_{41}(\varepsilon)$ is equal to the second term in $\eta_{4}$. We obtain the scalings $t \mapsto \varepsilon^{2} \eta_{3}(\varepsilon)+\varepsilon^{5 / 2} \eta_{4}(\varepsilon)+$ $\varepsilon^{5 / 2} t / \ln (1 / \varepsilon)^{1 / 2}$

$$
\begin{gathered}
T_{T} \mapsto T_{T}, \quad T_{B} \mapsto T_{B}, \quad T_{L} \mapsto T_{L}, \quad H_{P} \mapsto H_{P}, \quad H_{O} \mapsto \frac{\varepsilon^{5 / 2}}{\ln (1 / \varepsilon)^{1 / 2}} H_{O}, \quad R \mapsto R, \\
C \mapsto C, \quad I_{T} \mapsto \varepsilon I_{T}, \quad I_{B} \mapsto \frac{I_{B}}{\varepsilon}, \quad I_{L} \mapsto \varepsilon^{1 / 2} \overline{I_{L}}=\varepsilon^{1 / 2}\left(v \alpha_{2} \mu_{2} \Theta \eta_{4}+\frac{I_{L}}{\ln (1 / \varepsilon)^{1 / 2}}\right),
\end{gathered}
$$

and apply these to the system (2.8) leads to

$$
\begin{aligned}
\frac{d T_{T}}{d t}= & \frac{\varepsilon^{5 / 2}}{\ln (1 / \varepsilon)^{1 / 2}}\left(\delta_{T_{T}}\left(1-T_{T}\right)-\alpha_{4}\left(T_{T}-\mu_{4} T_{B}\right)\right) \\
\frac{d T_{B}}{d t}= & \frac{\varepsilon^{3 / 2}}{\ln (1 / \varepsilon)^{1 / 2}}\left(\alpha_{4}\left(T_{T}-\mu_{4} T_{B}\right)+\alpha_{1}\left(T_{L}-\mu_{1} T_{B}\right)-\varepsilon \delta_{T_{B}} T_{B}\right) \\
\frac{d T_{L}}{d t}= & \frac{\varepsilon^{1 / 2}}{\ln (1 / \varepsilon)^{1 / 2}}\left(S_{1}+k_{1} H_{P}-k_{-1} T_{L}-\varepsilon v \alpha_{1}\left(T_{L}-\mu_{1} T_{B}\right)-\varepsilon^{2} \delta_{T_{L}} T_{L}\right) \\
\frac{d H_{P}}{d t}= & \frac{\varepsilon^{1 / 2}}{\ln (1 / \varepsilon)^{1 / 2}}\left(k_{-1} T_{L}-k_{1} H_{P}-\varepsilon^{2} \delta_{P} H_{P}-\frac{\varepsilon^{5 / 2}}{\ln (1 / \varepsilon)^{1 / 2}} \delta_{H} H_{O} H_{P}\right) \\
\frac{d H_{O}}{d t}= & -2 \sqrt{k_{2} v \alpha_{2} \mu_{2} \Theta} H_{O}+\delta_{O}+k_{-2} C-\frac{k_{2} v \alpha_{2} \mu_{2} \Theta \eta_{41}}{\ln (1 / \varepsilon)^{1 / 2}} H_{O}-\frac{k_{2}}{\ln (1 / \varepsilon)} H_{O} I_{L} \\
\frac{d R}{d t}= & \frac{\varepsilon^{5 / 2}}{\ln (1 / \varepsilon)^{1 / 2}}\left(-\delta_{R} R+\frac{\varepsilon^{5 / 2}}{\ln (1 / \varepsilon)^{1 / 2}} \delta_{H} H_{O} H_{P}\right) \\
\frac{d C}{d t}= & \frac{\varepsilon^{5 / 2}}{\ln (1 / \varepsilon)^{1 / 2}}\left(2 \sqrt{k_{2} v \alpha_{2} \mu_{2} \Theta} H_{O}-\left(\delta_{C}+k_{-2}\right) C+\frac{k_{2} v \alpha_{2} \mu_{2} \Theta \eta_{41}}{\ln (1 / \varepsilon)^{1 / 2}} H_{O}+\frac{k_{2}}{\ln (1 / \varepsilon)} H_{O} I_{L}\right) \\
\frac{d I_{T}}{d t}= & \frac{\varepsilon^{1 / 2}}{\ln (1 / \varepsilon)^{1 / 2}}\left(\alpha_{3} \mu_{3} I_{B}-\varepsilon^{2}\left(\alpha_{3}+\delta_{I_{T}}\right) I_{T}\right) \\
\frac{d I_{B}}{d t}= & \frac{\varepsilon^{3 / 2}}{\ln (1 / \varepsilon)^{1 / 2}}\left(-\left(\alpha_{2} \mu_{2}+\alpha_{3} \mu_{3}\right) I_{B}-\varepsilon I_{B}+\varepsilon^{3 / 2} \alpha_{2} \overline{I_{L}}+\varepsilon^{2} \alpha_{3} I_{T}\right) \\
\frac{d I_{L}}{d t}= & v \alpha_{2} \mu_{2} I_{B}-\varepsilon^{3 / 2} v \alpha_{2} \overline{I_{L}}-\varepsilon^{2}\left(k_{2} \bar{I}_{L} H_{O}-k_{-2} C\right)-\varepsilon^{5 / 2} \delta_{I_{L}} \overline{I_{L}}
\end{aligned}
$$


As with the previous timescale:

$$
\begin{gathered}
C_{0}=1, T_{T_{0}}=T_{T_{0}}^{S S}, T_{B_{0}}=T_{B_{0}}^{S S}, I_{B_{0}}=\Theta, I_{T_{0}}=\frac{\alpha_{3} \mu_{3}}{\alpha_{2} \mu_{2} v} \\
T_{L_{0}}=T_{L_{0}}^{[2]}\left(\eta_{3}\right), H_{P_{0}}=H_{P_{0}}^{[2]}\left(\eta_{3}\right), R_{0}=R_{0}^{[2]}\left(\eta_{3}\right),
\end{gathered}
$$

and solving the ODEs for $H_{O_{0}}$ and $I_{L_{0}}$ gives

$$
H_{O_{0}}=\frac{\delta_{O}+k_{-2}}{2 \sqrt{k_{2} v \alpha_{2} \mu_{2} \Theta}}+\sqrt{\frac{v \alpha_{2} \mu_{2} \Theta}{2 \pi k_{2}}} e^{-2 \sqrt{k_{2} v \alpha_{2} \mu_{2} \Theta} t}, \quad I_{L_{0}}=v \alpha_{2} \mu_{2} \Theta t+c_{1}
$$

where $c_{1}$ is a constant of integration that requires further terms in the expansion of Timescale 3 to determine by matching.

All variables to leading order are constant other than $H_{O_{0}}$, which tends to a constant, and $I_{L_{0}}$ that grows linearly. This continues until liver inhibitor concentration accumulate to $I_{L_{0}}=O\left(\ln (1 / \varepsilon) / \varepsilon^{1 / 2}\right)$, leading to a small balance shift in the next timescale.

Timescale 5: $t=\varepsilon^{2} \eta_{3}(\varepsilon)+O\left(\varepsilon^{5 / 2} \ln (1 / \varepsilon)^{1 / 2}\right)$

Corresponds to about $t=O(2-3)$ mins. We introduce the scalings: $\quad t \mapsto \varepsilon^{2} \eta_{3}(\varepsilon)+\varepsilon^{5 / 2} \ln (1 / \varepsilon)^{1 / 2} t$,

$$
\begin{gathered}
T_{T} \mapsto T_{T}, \quad T_{B} \mapsto T_{B}, \quad T_{L} \mapsto T_{L}, \quad H_{P} \mapsto H_{P}, \quad H_{O} \mapsto \frac{\varepsilon^{5 / 2}}{\ln (1 / \varepsilon)^{1 / 2}} H_{O}, \\
R \mapsto R, \quad C \mapsto C, \quad I_{T} \mapsto \varepsilon I_{T}, \quad I_{B} \mapsto \frac{I_{B}}{\varepsilon}, \quad I_{L} \mapsto \varepsilon^{1 / 2} \ln (1 / \varepsilon)^{1 / 2} I_{L},
\end{gathered}
$$

and apply these to the system (2.8) gives

$$
\begin{aligned}
\frac{d T_{T}}{d t} & =\varepsilon^{5 / 2} \ln (1 / \varepsilon)^{1 / 2}\left(\delta_{T_{T}}\left(1-T_{T}\right)-\alpha_{4}\left(T_{T}-\mu_{4} T_{B}\right)\right) \\
\frac{d T_{B}}{d t} & =\varepsilon^{3 / 2} \ln (1 / \varepsilon)^{1 / 2}\left(\alpha_{4}\left(T_{T}-\mu_{4} T_{B}\right)+\alpha_{1}\left(T_{L}-\mu_{1} T_{B}\right)-\varepsilon \delta_{T_{B}} T_{B}\right) \\
\frac{d T_{L}}{d t} & =\varepsilon^{1 / 2} \ln (1 / \varepsilon)^{1 / 2}\left(S_{1}+k_{1} H_{P}-k_{-1} T_{L}-\varepsilon v \alpha_{1}\left(T_{L}-\mu_{1} T_{B}\right)-\varepsilon^{2} \delta_{T_{L}} T_{L}\right) \\
\frac{d H_{P}}{d t} & =\varepsilon^{1 / 2} \ln (1 / \varepsilon)^{1 / 2}\left(k_{-1} T_{L}-k_{1} H_{P}-\frac{\varepsilon^{5 / 2}}{\ln (1 / \varepsilon)^{1 / 2}} \delta_{H} H_{O} H_{P}-\varepsilon^{2} \delta_{P} H_{P}\right) \\
\frac{1}{\ln (1 / \varepsilon)} \frac{d H_{O}}{d t} & =-k_{2} H_{O} I_{L}+\delta_{O}+k_{-2} C-\frac{\varepsilon^{5 / 2}}{\ln (1 / \varepsilon)^{1 / 2}} \delta_{O} H_{O} \\
\frac{d R}{d t} & =\varepsilon^{1 / 2} \ln (1 / \varepsilon)^{1 / 2}\left(-\delta_{R} R+\frac{\varepsilon^{5 / 2}}{\ln (1 / \varepsilon)} \delta_{H} H_{O} H_{P}\right) \\
\frac{d C}{d t} & =\varepsilon^{5 / 2} \ln (1 / \varepsilon)^{1 / 2}\left(k_{2} H_{O} I_{L}-\left(\delta_{C}+k_{-2}\right) C\right) \\
\frac{d I_{T}}{d t} & =\varepsilon^{1 / 2} \ln (1 / \varepsilon)^{1 / 2}\left(\alpha_{3} \mu_{3} I_{B}-\varepsilon^{2}\left(\alpha_{3}+\delta_{I_{T}}\right) I_{T}\right)
\end{aligned}
$$


38 of 55

REFERENCES

$$
\begin{aligned}
& \frac{d I_{B}}{d t}=\varepsilon^{3 / 2} \ln (1 / \varepsilon)^{1 / 2}\left(-\left(\alpha_{2} \mu_{2}+\alpha_{3} \mu_{3}\right) I_{B}-\varepsilon I_{B}+\varepsilon^{3 / 2} \ln (1 / \varepsilon)^{1 / 2} \alpha_{2} I_{L}+\varepsilon^{2} \alpha_{3} I_{T}\right), \\
& \frac{d I_{L}}{d t}=v \alpha_{2} \mu_{2} I_{B}-\varepsilon^{3 / 2} \ln (1 / \varepsilon)^{1 / 2} v \alpha_{2} I_{L}-\varepsilon^{2}\left(k_{2} H_{O} I_{L}-k_{-2} C\right)-\varepsilon^{5 / 2} \ln (1 / \varepsilon)^{1 / 2} \delta_{I_{L}} I_{L} .
\end{aligned}
$$

To leading order

$$
\begin{gathered}
C_{0}=1, T_{T_{0}}=T_{T_{0}}^{S S}, T_{B_{0}}=T_{B_{0}}^{S S}, I_{B_{0}}=\Theta, I_{T_{0}}=\frac{\alpha_{3} \mu_{3}}{\alpha_{2} \mu_{2} v}, \\
T_{L_{0}}=T_{L_{0}}^{[2]}\left(\eta_{3}\right), H_{P_{0}}=H_{P_{0}}^{[2]}\left(\eta_{3}\right), R_{0}=R_{0}^{[2]}\left(\eta_{3}\right),
\end{gathered}
$$

and, by matching as $t \rightarrow 0^{+}$,

$$
I_{L_{0}}=v \alpha_{2} \mu_{2} \Theta t+2 \sqrt{\frac{v \alpha_{2} \mu_{2} \Theta}{k_{2}}}, \quad H_{O_{0}}=\frac{\delta_{O}+k_{-2}}{k_{2} v \alpha_{2} \mu_{2} \Theta t+2 \sqrt{k_{2} v \alpha_{2} \mu_{2} \Theta}} .
$$

The inhibitor continues to accumulate in the liver and HPPD continues to decline. There is a shift in balance in the equations when $t=O\left(\varepsilon^{-1 / 2} \ln (1 / \varepsilon)^{-1 / 2}\right)$.

Timescale 6: $t=\varepsilon^{2} \eta_{3}(\varepsilon)+O\left(\varepsilon^{2}\right)$

Corresponds to about $t=O(3-5)$ mins. We introduce the scalings $t \mapsto \varepsilon^{2} \eta_{3}(\varepsilon)+\varepsilon^{2} t$,

$$
\begin{aligned}
& T_{T} \mapsto T_{T}, \quad T_{B} \mapsto T_{B}, \quad T_{L} \mapsto T_{L}, \quad H_{P} \mapsto H_{P}, \quad H_{O} \mapsto \varepsilon^{3} H_{O}, \\
& R \mapsto R, \quad C \mapsto C, \quad I_{T} \mapsto \varepsilon I_{T}, \quad I_{B} \mapsto \frac{I_{B}}{\varepsilon}, \quad I_{L} \mapsto I_{L},
\end{aligned}
$$

and apply to the equations (2.8) resulting in

$$
\begin{aligned}
\frac{d T_{T}}{d t} & =\varepsilon^{2}\left(\delta_{T_{T}}\left(1-T_{T}\right)-\alpha_{4}\left(T_{T}-\mu_{4} T_{B}\right)\right), \\
\frac{d T_{B}}{d t} & =\varepsilon\left(\alpha_{4}\left(T_{T}-\mu_{4} T_{B}\right)+\alpha_{1}\left(T_{L}-\mu_{1} T_{B}\right)\right)-\varepsilon^{2} \delta_{T_{B}} T_{B}, \\
\frac{d T_{L}}{d t} & =S_{1}+k_{1} H_{P}-k_{-1} T_{L}-\varepsilon v \alpha_{1}\left(T_{L}-\mu_{1} T_{B}\right)-\varepsilon^{2} \delta_{T_{L}} T_{L}, \\
\frac{d H_{P}}{d t} & =-k_{1} H_{P}+k_{-1} T_{L}-\varepsilon^{2} \delta_{P} H_{P}-\varepsilon^{3} \delta_{H} H_{O} H_{P}, \\
\varepsilon \frac{d H_{O}}{d t} & =-k_{2} H_{O} I_{L}+\delta_{O}+k_{-2} C-\varepsilon^{3} \delta_{O} H_{O}, \\
\frac{d R}{d t} & =-\delta_{R} R+\varepsilon^{3} \delta_{H} H_{O} H_{P}, \\
\frac{d C}{d t} & =\varepsilon^{2}\left(k_{2} H_{O} I_{L}-\left(\delta_{C}+k_{-2}\right) C\right), \\
\frac{d I_{T}}{d t} & =\alpha_{3} \mu_{3} I_{B}-\varepsilon^{2}\left(\alpha_{3}+\delta_{I_{T}}\right) I_{T},
\end{aligned}
$$




$$
\begin{aligned}
& \frac{d I_{B}}{d t}=-\varepsilon\left(\alpha_{2} \mu_{2}+\alpha_{3} \mu_{3}\right) I_{B}+\varepsilon^{2}\left(\alpha_{2} I_{L}-I_{B}\right)+\varepsilon^{3} \alpha_{3} I_{T}, \\
& \frac{d I_{L}}{d t}=v \alpha_{2} \mu_{2} I_{B}-\varepsilon \alpha_{2} v I_{L}-\varepsilon^{2}\left(k_{2} H_{O} I_{L}-k_{-2} C+\delta_{I_{L}} I_{L}\right) .
\end{aligned}
$$

At leading order we have

$$
C_{0}=1, \quad T_{T_{0}}=T_{T_{0}}^{S S}, \quad T_{B_{0}}=T_{B_{0}}^{S S}, \quad I_{B_{0}}=\Theta
$$

and

$$
\begin{aligned}
I_{T_{0}} & =\frac{\alpha_{3} \mu_{3}}{\alpha_{2} \mu_{2} v}+\alpha_{3} \mu_{3} \Theta t, I_{L_{0}}=v \alpha_{2} \mu_{2} \Theta t, H_{O_{0}}=\frac{\delta_{O}+k_{-2}}{k_{2} v \alpha_{2} \mu_{2} \Theta t}, R_{0}=R_{0}^{[2]}\left(\eta_{3}\right) e^{-\delta_{R} t} \\
H_{P_{0}} & =H_{P_{0}}^{[2]}\left(\eta_{3}\right)+\frac{k_{-1} S_{1}}{k_{1}+k_{-1}} t+\frac{\left(1-e^{-\left(k_{1}+k_{-1}\right) t}\right)}{k_{1}+k_{-1}}\left(k_{1} T_{L_{0}}^{[2]}\left(\eta_{3}\right)-k_{-1} H_{P_{0}}^{[2]}\left(\eta_{3}\right)-\frac{k_{-1} S_{1}}{k_{1}+k_{-1}}\right) \\
T_{L_{0}} & =T_{L_{0}}^{[2]}\left(\eta_{3}\right)+\frac{k_{1} S_{1}}{k_{1}+k_{-1}} t-\frac{\left(1-e^{-\left(k_{1}+k_{-1}\right) t}\right)}{k_{1}+k_{-1}}\left(k_{1} T_{L_{0}}^{[2]}\left(\eta_{3}\right)-k_{-1} H_{P_{0}}^{[2]}\left(\eta_{3}\right)-\frac{k_{-1} S_{1}}{k_{1}+k_{-1}}\right) .
\end{aligned}
$$

Here, the homogentisic acid and downstream products drop rapidly, and since in large time $H_{O} H_{P}=$ $O(1)$, there is a shift in balance in the $R$ equation at $t=\left(3 / \delta_{H}\right) \ln (1 / \varepsilon)+O(1)$ when $R=O\left(\varepsilon^{3}\right)$.

Timescale 7: $t=\varepsilon^{2}\left(\eta_{3}(\varepsilon)+\eta_{7}(\varepsilon)\right)+O\left(\varepsilon^{2}\right)$

Corresponds to about $t=O(5-8)$ mins. Here, $\eta_{7}(\varepsilon)=\left(3 / \delta_{H}\right) \ln (1 / \varepsilon)$ we obtain the scalings $t \mapsto$ $\varepsilon^{2}\left(\eta_{3}(\varepsilon)+\eta_{7}(\varepsilon)\right)+\varepsilon^{2} t$ and

$$
\begin{gathered}
T_{T} \mapsto T_{T}, \quad T_{B} \mapsto T_{B}, \quad T_{L} \mapsto \overline{T_{L}}=\eta_{7} T_{L}^{C}+T_{L}, \\
H_{P} \mapsto \overline{H_{P}}=\eta_{7} \frac{k_{-1} T_{L}^{C}}{k_{1}}+H_{P}, \quad H_{O} \mapsto \varepsilon^{3} \overline{H_{O}}=\varepsilon^{3}\left(\frac{H_{O}^{C}}{\eta_{7}}+\frac{H_{O}}{\ln (1 / \varepsilon)^{2}}\right), \quad R \mapsto \varepsilon^{3} R, \\
C \mapsto C, \quad I_{T} \mapsto \varepsilon \overline{I_{T}}=\varepsilon\left(\eta_{7} I_{T}^{C}+I_{T}\right), \quad I_{B} \mapsto \frac{I_{B}}{\varepsilon}, \quad I_{L} \mapsto \overline{I_{L}}=\eta_{7} I_{L}^{C}+I_{L},
\end{gathered}
$$

where $T_{L}^{C}=k_{1} S_{1} /\left(k_{1}+k_{-1}\right), H_{O}^{C}=\left(\delta_{O}+k_{-2}\right) /\left(k_{2} v \alpha_{2} \mu_{2} \Theta\right), I_{T}^{C}=\alpha_{3} \mu_{3} \Theta$ and $I_{L}^{C}=v \alpha_{2} \mu_{2} \Theta$. Applying these to (2.8) we obtain

$$
\begin{aligned}
\frac{d T_{T}}{d t} & =\varepsilon^{2}\left(\delta_{T_{T}}\left(1-T_{T}\right)-\alpha_{4}\left(T_{T}-\mu_{4} T_{B}\right)\right) \\
\frac{d T_{B}}{d t} & =\varepsilon\left(\alpha_{4}\left(T_{T}-\mu_{4} T_{B}\right)+\alpha_{1}\left(\overline{T_{L}}-\mu_{1} T_{B}\right)\right)-\varepsilon^{2} \delta_{T_{B}} T_{B}, \\
\frac{d T_{L}}{d t} & =S_{1}+k_{1} H_{P}-k_{-1} T_{L}-\varepsilon v \alpha_{1}\left(\overline{T_{L}}-\mu_{1} T_{B}\right)-\varepsilon^{2} \delta_{T_{L}} \overline{T_{L}}, \\
\frac{d H_{P}}{d t} & =-k_{1} H_{P}+k_{-1} T_{L}-\varepsilon^{2} \delta_{P} \overline{H_{P}}-\varepsilon^{3} \delta_{H} \overline{H_{O}} \overline{H_{P}} \\
\frac{\varepsilon}{\ln (1 / \varepsilon)^{2}} \frac{d H_{O}}{d t} & =-k_{2} H_{O}^{C} I_{L}^{C}+\delta_{O}+k_{-2} C-\frac{k_{2}}{\eta_{7}} H_{O}^{C} I_{L}-\frac{k_{2}}{\ln (1 / \varepsilon)^{2}} H_{O} \overline{I_{L}}-\varepsilon^{3} \delta_{O} \overline{H_{O}}
\end{aligned}
$$


40 of 55

REFERENCES

$$
\begin{aligned}
\frac{d R}{d t} & =\frac{\delta_{H} k_{-1}}{k_{1}} T_{L}^{C} H_{O}^{C}-\delta_{R} R+\frac{\delta_{H}}{\eta_{7}} H_{O}^{C} H_{P}+\frac{\delta_{H}}{\ln (1 / \varepsilon)^{2}} H_{O} \overline{H_{P}} \\
\frac{d C}{d t} & =\varepsilon^{2}\left(k_{2} \overline{H_{O}} \overline{I_{L}}-\left(\delta_{C}+k_{-2}\right) C\right) \\
\frac{d I_{T}}{d t} & =\alpha_{3} \mu_{3} I_{B}-\varepsilon^{2}\left(\alpha_{3}+\delta_{I_{T}}\right) \overline{I_{T}} \\
\frac{d I_{B}}{d t} & =-\varepsilon\left(\alpha_{2} \mu_{2}+\alpha_{3} \mu_{3}\right) I_{B}+\varepsilon^{2}\left(\alpha_{2} \overline{I_{L}}-I_{B}\right)+\varepsilon^{3} \alpha_{3} \overline{I_{T}} \\
\frac{d I_{L}}{d t} & =v \alpha_{2} \mu_{2} I_{B}-\varepsilon \alpha_{2} v \overline{I_{L}}-\varepsilon^{2}\left(k_{2} \overline{H_{O}} \overline{I_{L}}-k_{-2} C+\delta_{I_{L}} \overline{I_{L}}\right) .
\end{aligned}
$$

All variables except $H_{P_{0}}, T_{L_{0}}$ and $R_{0}$ have the same leading order expansions as those of Timescale 6. The exponential decay terms in $H_{P_{0}}$ and $T_{L_{0}}$ in the previous timescale have become negligible, hence,

$$
\begin{aligned}
& H_{P_{0}}=H_{P_{0}}^{[2]}\left(\eta_{3}\right)+\frac{k_{-1} S_{1}}{k_{1}+k_{-1}} t+\frac{1}{k_{1}+k_{-1}}\left(k_{1} T_{L_{0}}^{[2]}\left(\eta_{3}\right)-k_{-1} H_{P_{0}}^{[2]}\left(\eta_{3}\right)-\frac{k_{-1} S_{1}}{k_{1}+k_{-1}}\right) \\
& T_{L_{0}}=T_{L_{0}}^{[2]}\left(\eta_{3}\right)+\frac{k_{1} S_{1}}{k_{1}+k_{-1}} t-\frac{1}{k_{1}+k_{-1}}\left(k_{1} T_{L_{0}}^{[2]}\left(\eta_{3}\right)-k_{-1} H_{P_{0}}^{[2]}\left(\eta_{3}\right)-\frac{k_{-1} S_{1}}{k_{1}+k_{-1}}\right)
\end{aligned}
$$

Finally,

$$
R_{0}=\frac{S_{1} \delta_{H} k_{-1}\left(\delta_{O}+k_{-2}\right)}{\delta_{R}\left(k_{1}+k_{-1}\right) k_{2} v \alpha_{2} \mu_{2} \Theta}+R_{0}^{[2]}\left(\eta_{3}\right) e^{-\delta_{R} t}=R_{\min }^{[7]}+R_{0}^{[2]}\left(\eta_{3}\right) e^{-\delta_{R} t} .
$$

On this timescale homogentisic acid reaches its minimum level, while HPPA and liver tyrosine continues to rise. The linear growth of $I_{T}, I_{L}, H_{P}$ and $T_{L}$ and decay of $H_{O_{0}}$ means that a new balance in equations will occur when $t=O\left(\varepsilon^{-1 / 2}\right)$.

Timescale 8: $t=O\left(\varepsilon^{3 / 2}\right)$

Corresponds to about $t=O(8)$ mins. We introduce the scalings $t \mapsto \varepsilon^{3 / 2} t$ and

$$
\begin{aligned}
& T_{T} \mapsto T_{T}, \quad T_{B} \mapsto T_{B}, \quad T_{L} \mapsto \frac{T_{L}}{\varepsilon^{1 / 2}}, \quad H_{P} \mapsto \frac{H_{P}}{\varepsilon^{1 / 2}}, \quad H_{O} \mapsto \varepsilon^{7 / 2} H_{O}, \\
& R \mapsto \varepsilon^{3} R, \quad C \mapsto C, \quad I_{T} \mapsto \varepsilon^{1 / 2} I_{T}, \quad I_{B} \mapsto \frac{I_{B}}{\varepsilon}, \quad I_{L} \mapsto \frac{I_{L}}{\varepsilon^{1 / 2}},
\end{aligned}
$$

applying them to the system (2.8) resulting in

$$
\begin{aligned}
\frac{d T_{T}}{d t} & =\varepsilon^{3 / 2}\left(\delta_{T_{T}}\left(1-T_{T}\right)-\alpha_{4}\left(T_{T}-\mu_{4} T_{B}\right)\right), \\
\frac{d T_{B}}{d t} & =\alpha_{1} T_{L}-\varepsilon^{1 / 2}\left(\alpha_{1} \mu_{1} T_{B}-\alpha_{4}\left(T_{T}-\mu_{4} T_{B}\right)\right)-\varepsilon^{3 / 2} \delta_{T_{B}} T_{B}, \\
\varepsilon^{1 / 2} \frac{d T_{L}}{d t} & =k_{1} H_{P}-k_{-1} T_{L}+\varepsilon^{1 / 2} S_{1}-\varepsilon v \alpha_{1} T_{L}+\varepsilon^{3 / 2} v \alpha_{1} \mu_{1} T_{B}-\varepsilon^{2} \delta_{T_{L}} T_{L}, \\
\varepsilon^{1 / 2} \frac{d H_{P}}{d t} & =-k_{1} H_{P}+k_{-1} T_{L}-\varepsilon^{2} \delta_{P} H_{P}-\varepsilon^{7 / 2} \delta_{H} H_{O} H_{P},
\end{aligned}
$$




$$
\begin{aligned}
\varepsilon^{2} \frac{d H_{O}}{d t} & =-k_{2} H_{O} I_{L}+\delta_{O}+k_{-2} C-\varepsilon^{7 / 2} \delta_{O} H_{O}, \\
\varepsilon^{1 / 2} \frac{d R}{d t} & =\delta_{H} H_{O} H_{P}-\delta_{R} R, \\
\frac{d C}{d t} & =\varepsilon^{3 / 2}\left(k_{2} H_{O} I_{L}-\left(\delta_{C}+k_{-2}\right) C\right), \\
\frac{d I_{T}}{d t} & =\alpha_{3} \mu_{3} I_{B}-\varepsilon^{3 / 2}\left(\alpha_{3}+\delta_{I_{T}}\right) I_{T}, \\
\frac{d I_{B}}{d t} & =-\varepsilon^{1 / 2}\left(\alpha_{2} \mu_{2}+\alpha_{3} \mu_{3}\right) I_{B}+\varepsilon \alpha_{2} I_{L}-\varepsilon^{3 / 2} I_{B}+\varepsilon^{2} \alpha_{3} I_{T}, \\
\frac{d I_{L}}{d t} & =v \alpha_{2} \mu_{2} I_{B}-\varepsilon^{1 / 2} v \alpha_{2} I_{L}-\varepsilon^{3 / 2} \delta_{I_{L}} I_{L}-\varepsilon^{2}\left(k_{2} H_{O} I_{L}-k_{-2} C\right) .
\end{aligned}
$$

The following are constant to leading order

$$
T_{T_{0}}=T_{T_{0}}^{S S}, \quad C_{0}=1, \quad I_{B}=\Theta,
$$

and the inhibitor variables decouple and solve to give

$$
I_{L_{0}}=v \alpha_{2} \mu_{2} \Theta t, \quad I_{T_{0}}=\alpha_{3} \mu_{3} \Theta t, \quad H_{O_{0}}=\frac{\delta_{O}+k_{-2}}{k_{2} v \alpha_{2} \mu_{2} \Theta t},
$$

Adding the 3 rd and 4 th ODE with $\varepsilon v \times$ the 2 nd leads to

$$
\frac{d\left(H_{P_{0}}+T_{L_{0}}\right)}{d t}=S_{1}
$$

so $H_{P_{0}}+T_{L_{0}}=S_{1} t$ by matching as $t \rightarrow 0^{+}$. Since $H_{P_{0}}=k_{-1} T_{L_{0}} / k_{1}$ then

$$
T_{L_{0}}=\frac{S_{1} k_{1}}{k_{1}+k_{-1}} t, \quad H_{P_{0}}=\frac{S_{1} k_{-1}}{k_{1}+k_{-1}} t
$$

and hence

$$
T_{B_{0}}=T_{B_{0}}^{S S}+\frac{S_{1} k_{1} \alpha_{1}}{2\left(k_{1}+k_{-1}\right)} t^{2}, \quad R_{0}=R_{\text {min }}^{[7]} .
$$

The inhibitor continues to increase in the liver (and tissue) and consequently HPPA increases and HPPD continues to decrease. The increasing HPPA leads to liver tyrosine accumulating sufficiently in the liver to affect the blood concentration. The solutions are valid until $T_{B}=O\left(T_{L}\right)$ at $t=O\left(\varepsilon^{-1 / 2}\right)$.

Timescale 9: $t=O(\varepsilon)$

Corresponds to about $t=O(40-60)$ mins. On this timescale we rescale time such that $t \mapsto \varepsilon t$ and introduce the scalings

$$
\begin{aligned}
& T_{T} \mapsto T_{T}, \quad T_{B} \mapsto \frac{T_{B}}{\varepsilon}, \quad T_{L} \mapsto \frac{T_{L}}{\varepsilon}, \quad H_{P} \mapsto \frac{H_{P}}{\varepsilon}, \quad H_{O} \mapsto \varepsilon^{4} H_{O}, \\
& R \mapsto \varepsilon^{3} R, \quad C \mapsto C, \quad I_{T} \mapsto I_{T}, \quad I_{B} \mapsto \frac{I_{B}}{\varepsilon}, \quad I_{L} \mapsto \frac{I_{L}}{\varepsilon},
\end{aligned}
$$


which are applied to the equations (2.8) leads to

$$
\begin{aligned}
\frac{d T_{T}}{d t} & =\alpha_{4} \mu_{4} T_{B}+\varepsilon\left(\delta_{T_{T}}\left(1-T_{T}\right)-\alpha_{4} T_{T}\right) \\
\frac{d T_{B}}{d t} & =\alpha_{1} T_{L}-\left(\alpha_{1} \mu_{1}+\alpha_{4} \mu_{4}\right) T_{B}+\varepsilon\left(\alpha_{4} T_{T}-\delta_{T_{B}} T_{B}\right) \\
\varepsilon \frac{d T_{L}}{d t} & =k_{1} H_{P}-k_{-1} T_{L}+\varepsilon\left(S_{1}-v \alpha_{1} T_{L}+v \alpha_{1} \mu_{1} T_{B}\right)-\varepsilon^{2} \delta_{T_{L}} T_{L} \\
\varepsilon \frac{d H_{P}}{d t} & =-k_{1} H_{P}+k_{-1} T_{L}-\varepsilon^{2} \delta_{P} H_{P}-\varepsilon^{4} \delta_{H} H_{O} H_{P} \\
\varepsilon \frac{d H_{O}}{d t} & =-k_{2} H_{O} I_{L}+\delta_{O}+k_{-2} C-\varepsilon^{4} \delta_{O} H_{O} \\
\varepsilon \frac{d R}{d t} & =\delta_{H} H_{O} H_{P}-\delta_{R} R \\
\frac{d C}{d t} & =\varepsilon\left(k_{2} H_{O} I_{L}-\left(\delta_{C}+k_{-2}\right) C\right) \\
\frac{d I_{T}}{d t} & =\alpha_{3} \mu_{3} I_{B}-\varepsilon\left(\alpha_{3}+\delta_{I_{T}}\right) I_{T}, \\
\frac{d I_{B}}{d t} & =-\left(\alpha_{2} \mu_{2}+\alpha_{3} \mu_{3}\right) I_{B}+\alpha_{2} I_{L}+\varepsilon\left(\alpha_{3} I_{T}-I_{B}\right) \\
\frac{d I_{L}}{d t} & =v \alpha_{2}\left(\mu_{2} I_{B}-I_{L}\right)-\varepsilon \delta_{I_{L}} I_{L}-\varepsilon^{2}\left(k_{2} H_{O} I_{L}-k_{-2} C\right)
\end{aligned}
$$

We have the quasi-steady solutions

$$
C_{0}=1, \quad H_{P_{0}}=\frac{k_{-1}}{k_{1}} T_{L_{0}}, \quad H_{O_{0}}=\frac{\delta_{O}+k_{-2}}{k_{2} I_{L_{0}}}
$$

where $T_{L_{0}}$ and $I_{L_{0}}$ are derived below. At leading order $H_{P_{0}}=k_{-1} T_{L_{0}} / k_{1}$. Adding the equations for $T_{L}$ and $H_{P}$ leads to the system

$$
\begin{aligned}
\frac{d T_{T_{0}}}{d t}= & \alpha_{4} \mu_{4} T_{B_{0}}, \quad \frac{d T_{B_{0}}}{d t}=\alpha_{1} T_{L_{0}}-\left(\alpha_{1} \mu_{1}+\alpha_{4} \mu_{4}\right) T_{B_{0}} \\
& \left(1+\frac{k_{-1}}{k_{1}}\right) \frac{d T_{L_{0}}}{d t}=S_{1}-\alpha_{1}\left(T_{L_{0}}-\mu_{1} T_{B_{0}}\right)
\end{aligned}
$$

the latter two ODEs reduce to

$$
\frac{d^{2} T_{B_{0}}}{d t^{2}}+\left(\frac{k_{1} v \alpha_{1}}{k_{1}+k_{-1}}+\alpha_{1} \mu_{1}+\alpha_{4} \mu_{4}\right) \frac{d T_{B_{0}}}{d t}+\frac{k_{1} v \alpha_{1} \alpha_{4} \mu_{4}}{k_{1}+k_{-1}} T_{B_{0}}=\frac{S_{1} k_{1} \alpha_{1}}{k_{1}+k_{-1}}
$$


subject to, by matching, $T_{B_{0}}=d T_{B_{0}} / d t=0$ at $t=0$. This solves to give

$$
\begin{aligned}
T_{B_{0}}= & \frac{S_{1}}{v \alpha_{4} \mu_{4}}\left(1+\frac{\omega^{-} e^{\omega^{+} t}}{\omega^{+}-\omega^{-}}-\frac{\omega^{+} e^{\omega^{-} t}}{\omega^{+}-\omega^{-}}\right) \\
T_{T_{0}}= & T_{T_{0}}^{S S}+\frac{S_{1}}{v}\left(t-\frac{\omega^{-}\left(1-e^{\omega^{+} t}\right)}{\omega^{+}\left(\omega^{+}-\omega^{-}\right)}+\frac{\omega^{+}\left(1-e^{\omega^{-} t}\right)}{\omega^{-}\left(\omega^{+}-\omega^{-}\right)}\right), \\
T_{L_{0}}= & \frac{S_{1}\left(\alpha_{1} \mu_{1}+\alpha_{4} \mu_{4}\right)}{v \alpha_{1} \alpha_{4} \mu_{4}}+\frac{S_{1}}{v \alpha_{1} \alpha_{4} \mu_{4}\left(\omega^{+}-\omega^{-}\right)} \\
& \left(\omega^{-}\left(\alpha_{1} \mu_{1}+\alpha_{4} \mu_{4}+\omega^{+}\right) e^{\omega^{+} t}+\omega^{+}\left(\alpha_{1} \mu_{1}+\alpha_{4} \mu_{4}+\omega^{-}\right) e^{\omega^{-} t}\right),
\end{aligned}
$$

where

$$
\omega^{ \pm}=-\frac{1}{2}\left(\frac{k_{1} v \alpha_{1}}{k_{1}+k_{-1}}+\alpha_{1} \mu_{1}+\alpha_{4} \mu_{4}\right) \pm \frac{1}{2}\left(\left(\frac{k_{1} v \alpha_{1}}{k_{1}+k_{-1}}+\alpha_{1} \mu_{1}-\alpha_{4} \mu_{4}\right)^{2}+4 \alpha_{1} \mu_{1} \alpha_{4} \mu_{4}\right)^{1 / 2}
$$

and it can be shown that $\omega^{-}<\omega^{+}<0$.

The equations for the inhibitor are decoupled and combine to give

$$
\frac{d^{2} I_{B_{0}}}{d t^{2}}+\left(\alpha_{3} \mu_{3}+\alpha_{2}\left(\mu_{2}+v\right)\right) \frac{d I_{B_{0}}}{d t}+v \alpha_{2} \alpha_{3} \mu_{3} I_{B_{0}}=0
$$

which solves to

$$
\begin{aligned}
I_{L_{0}} & =\frac{\Theta v \alpha_{2} \mu_{2}}{\rho^{+}-\rho^{-}}\left(e^{\rho^{+} t}-e^{\rho^{-} t}\right), \\
I_{B_{0}} & =\frac{\Theta}{\rho^{+}-\rho^{-}}\left(\left(\rho^{+}+\alpha_{2} \mu_{2}+\alpha_{3} \mu_{3}\right) e^{\rho^{-} t}-\left(\rho^{-}+\alpha_{2} \mu_{2}+\alpha_{3} \mu_{3}\right) e^{\rho^{+} t}\right), \\
I_{T_{0}} & =\Theta\left(1+\frac{\left(\rho^{-}+\alpha_{2} \mu_{2}+\alpha_{3} \mu_{3}\right)\left(\rho^{+}+\alpha_{2}\left(\mu_{2}+v\right)+\alpha_{3} \mu_{3}\right)}{\alpha_{2} v\left(\rho^{+}-\rho^{-}\right)} e^{\rho^{+} t}\right. \\
& \left.-\frac{\left(\rho^{+}+\alpha_{2} \mu_{2}+\alpha_{3} \mu_{3}\right)\left(\rho^{-}+\alpha_{2}\left(\mu_{2}+v\right)+\alpha_{3} \mu_{3}\right)}{\alpha_{2} v\left(\rho^{+}-\rho^{-}\right)} e^{\rho^{-} t}\right)
\end{aligned}
$$

where

$$
\left.\rho^{ \pm}=-\frac{\left(\alpha_{3} \mu_{3}+\alpha_{2}\left(\mu_{2}+v\right)\right)}{2} \pm \frac{1}{2}\left(\alpha_{3} \mu_{3}+\alpha_{2}\left(\mu_{2}+v\right)\right)^{2}-4 v \alpha_{2} \alpha_{3} \mu_{3}\right)^{1 / 2}
$$

and that $\rho^{-}<\rho^{+}<0$. The remaining variable is

$$
R_{0}=\frac{\delta_{H} H_{O} H_{P}}{\delta_{R}}=\frac{\delta_{H} k_{1}\left(\delta_{O}+k_{-2}\right)}{\delta_{R} k_{2}} \frac{T_{L_{0}}}{I_{L_{0}}}
$$


Of particular note is behaviour as $t \rightarrow \infty$ namely,

$$
\begin{aligned}
T_{B_{0}} & \sim \frac{S_{1}}{v \alpha_{4} \mu_{4}}=T_{B_{\infty}}^{[9]}, \quad T_{T_{0}} \sim \frac{S_{1}}{v} t, \quad T_{L_{0}} \sim \frac{S_{1}\left(\alpha_{1} \mu_{1}+\alpha_{4} \mu_{4}\right)}{v \alpha_{1} \alpha_{4} \mu_{4}}=T_{L_{\infty}}^{[9]}, \\
I_{L_{0}} & \sim \frac{\Theta v \alpha_{2} \mu_{2}}{\rho^{+}-\rho^{-}} e^{\rho^{+} t}, \quad I_{B_{0}} \sim \frac{\Theta}{\rho^{+}-\rho^{-}}\left(-\left(\rho^{-}+\alpha_{2} \mu_{2}+\alpha_{3} \mu_{3}\right)\right) e^{\rho^{+} t}, \quad I_{T_{0}} \sim \Theta, \\
H_{P_{0}} & \sim \frac{S_{1} k_{1}\left(\alpha_{1} \mu_{1}+\alpha_{4} \mu_{4}\right)}{k_{-1} v \alpha_{1} \alpha_{4} \mu_{4}}=H_{P_{\infty}}^{[9]}, \quad H_{O_{0}} \sim \frac{\left(\delta_{O}+k_{-2}\right)\left(\rho^{+}-\rho^{-}\right)}{k_{2} v \alpha_{2} \mu_{2} \Theta} e^{-\rho^{+} t}, \\
R_{0} & \sim \frac{S_{1} \delta_{H} k_{1}\left(\alpha_{1} \mu_{1}+\alpha_{4} \mu_{4}\right)\left(\delta_{O}+k_{-2}\right)\left(\rho^{+}-\rho^{-}\right)}{\delta_{R} k_{-1} k_{2} v^{2} \alpha_{1} \alpha_{4} \mu_{4} \alpha_{2} \mu_{2} \Theta} e^{-\rho^{+} t} .
\end{aligned}
$$

Blood tyrosine has reached a (temporary) maximum and tissue concentrations are rising. Inhibitor concentration in the blood and eventually in the liver declines as it infiltrates into tissue acting as an inhibitor sink. As the liver inhibitor concentration declines, HPPD and homogentisic acid starts to rise. There is a shift in balance when $I_{B_{0}}=O(\varepsilon)$ and $I_{L_{0}}=O(\varepsilon)$, i.e. $t=\ln (1 / \varepsilon) /\left(-\rho^{+}\right)+O(1)$.

Timescale 10: $t=\varepsilon \eta_{10}(\varepsilon)+O(\varepsilon)$

Corresponds to about $t=O(5)$ hours. Here, $\eta_{10}(\varepsilon)=\ln (1 / \varepsilon) /\left(-\rho^{+}\right)$we scale time such that $t \mapsto$ $\varepsilon \eta_{10}(\varepsilon)+\varepsilon t$ and introduce

$$
\begin{aligned}
& T_{T} \mapsto \overline{T_{T}}=\eta_{10} \frac{S_{1}}{v}+T_{T}, T_{B} \mapsto \frac{T_{B}}{\varepsilon}, \\
& R \mapsto \varepsilon^{2} R, \quad C \mapsto \frac{T_{L}}{\varepsilon}, \quad H_{P} \mapsto \frac{H_{P}}{\varepsilon}, \quad H_{O} \mapsto \varepsilon^{3} H_{O}, \\
& R, \quad I_{T} \mapsto I_{T}, \quad I_{B} \mapsto I_{B}, \quad I_{L} \mapsto I_{L} .
\end{aligned}
$$

Applying these to the equations of the previous timescale we obtain

$$
\begin{aligned}
\frac{d T_{T}}{d t} & =\alpha_{4} \mu_{4} T_{B}+\varepsilon\left(\delta_{T_{T}}\left(1-\overline{T_{T}}\right)-\alpha_{4} \overline{T_{T}}\right), \\
\frac{d T_{B}}{d t} & =\alpha_{1} T_{L}-\left(\alpha_{1} \mu_{1}+\alpha_{4} \mu_{4}\right) T_{B}+\varepsilon\left(\alpha_{4} \overline{T_{T}}-\delta_{T_{B}} T_{B}\right), \\
\varepsilon \frac{d T_{L}}{d t} & =k_{1} H_{P}-k_{-1} T_{L}+\varepsilon\left(S_{1}-v \alpha_{1} T_{L}+v \alpha_{1} \mu_{1} T_{B}\right)-\varepsilon^{2} \delta_{T_{L}} T_{L}, \\
\varepsilon \frac{d H_{P}}{d t} & =-k_{1} H_{P}+k_{-1} T_{L}-\varepsilon^{2} \delta_{P} H_{P}-\varepsilon^{3} \delta_{H} H_{O} H_{P}, \\
\varepsilon^{2} \frac{d H_{O}}{d t} & =-k_{2} H_{O} I_{L}+\delta_{O}+k_{-2} C-\varepsilon^{3} \delta_{O} H_{O}, \\
\varepsilon \frac{d R}{d t} & =\delta_{H} H_{O} H_{P}-\delta_{R} R \\
\frac{d C}{d t} & =\varepsilon\left(k_{2} H_{O} I_{L}-\left(\delta_{C}+k_{-2}\right) C\right), \\
\frac{d I_{T}}{d t} & =\varepsilon\left(\alpha_{3}\left(I_{T}-\mu_{3} I_{B}\right)-\delta_{I_{T}} I_{T}\right), \\
\frac{d I_{B}}{d t} & =\alpha_{2}\left(I_{L}-\mu_{2} I_{B}\right)+\alpha_{3}\left(I_{T}-\mu_{3} I_{B}\right)-\varepsilon I_{B}, \\
\frac{d I_{L}}{d t} & =-v \alpha_{2}\left(I_{L}-\mu_{2} I_{B}\right)-\varepsilon\left(k_{2} H_{O} I_{L}-k_{-2} C+\delta_{I_{L}} I_{L}\right) .
\end{aligned}
$$


Through matching as $t \rightarrow-\infty$ we obtain

$$
C_{0}=1, \quad T_{B_{0}}=T_{B_{\infty}}^{[9]}, \quad T_{L_{0}}=T_{L_{\infty}}^{[9]}, \quad H_{P_{0}}=H_{P_{\infty}}^{[9]}, \quad I_{T_{0}}=\Theta,
$$

and we can deduce

$$
T_{T_{0}}=\frac{S_{1}}{v} t
$$

Constant $I_{T_{0}}$ leads to

$$
\frac{d^{2} I_{B_{0}}}{d t^{2}}+\left(\alpha_{3} \mu_{3}+\alpha_{2}\left(\mu_{2}+v\right)\right) \frac{d I_{B_{0}}}{d t}+v \alpha_{2} \alpha_{3} \mu_{3} I_{B_{0}}=v \alpha_{2} \alpha_{3} \Theta
$$

which on solution and matching yields

$$
\begin{aligned}
I_{B_{0}} & =\frac{\Theta}{\mu_{3}}-\Theta \frac{\left(\rho^{-}+\alpha_{2} \mu_{2}+\alpha_{3} \mu_{3}\right)}{\rho^{+}-\rho^{-}} e^{\rho^{+} t} \\
I_{L_{0}} & =\frac{\mu_{2} \Theta}{\mu_{3}}+\frac{\Theta v \alpha_{2} \mu_{2}}{\rho^{+}-\rho^{-}} e^{\rho^{+} t}
\end{aligned}
$$

and $\delta_{O}+k_{-2} C_{0}-k_{2} H_{O_{0}} I_{L_{0}}=0$ gives

$$
H_{O_{0}}=\frac{\mu_{3}\left(\rho^{+}-\rho^{-}\right)\left(\delta_{O}+k_{-2}\right)}{\Theta k_{2} \mu_{2}\left(\rho^{+}-\rho^{-}+v \alpha_{2} \mu_{3} e^{\rho^{+} t}\right)}
$$

and

$$
R_{0}=\frac{\delta_{H}}{\delta_{R}} H_{P_{\infty}}^{[9]} H_{O_{0}}
$$

The inhibitor concentration is equilibrating throughout as with all variables, except tissue tyrosine which is increasing. The HPPD concentration is still very low as is homogentisic acid. The continued increase of $T_{T_{0}}$ leads to a balance shift when $T_{T_{0}}=O(1 / \varepsilon)$, i.e. $t=O(1 / \varepsilon)$.

Timescale 11: $t=O(1)$

Corresponds to about $t=O(16-24)$ hours. On this timescale time is $O(1)$ and we introduce the scalings

$$
\begin{gathered}
T_{T} \mapsto \frac{T_{T}}{\varepsilon}, \quad T_{B} \mapsto \frac{T_{B}}{\varepsilon}, \quad T_{L} \mapsto \frac{T_{L}}{\varepsilon}, \quad H_{P} \mapsto \frac{H_{P}}{\varepsilon}, \\
R \mapsto \varepsilon^{2} R, \quad C \mapsto C, \quad I_{T} \mapsto I_{T}, \quad H_{B} \mapsto \varepsilon^{3} H_{O}, \\
R \mapsto I_{B}, \quad I_{L} \mapsto I_{L} .
\end{gathered}
$$

which are applied to the equations for the previous timescale

$$
\begin{aligned}
\frac{d T_{T}}{d t} & =-\delta_{T_{T}} T_{T}-\alpha_{4}\left(T_{T}-\mu_{4} T_{B}\right)+\varepsilon \delta_{T_{T}} \\
\varepsilon \frac{d T_{B}}{d t} & =\alpha_{1}\left(T_{L}-\mu_{1} T_{B}\right)+\alpha_{4}\left(T_{T}-\mu_{4} T_{B}\right)-\varepsilon \delta_{T_{B}} T_{B}
\end{aligned}
$$


46 of 55

REFERENCES

$$
\begin{aligned}
\varepsilon^{2} \frac{d T_{L}}{d t} & =k_{1} H_{P}-k_{-1} T_{L}+\varepsilon\left(S_{1}-\alpha_{1} v\left(T_{L}-\mu_{1} T_{B}\right)\right)-\varepsilon^{2} \delta_{T_{L}} T_{L}, \\
\varepsilon^{2} \frac{d H_{P}}{d t} & =-k_{1} H_{P}+k_{-1} T_{L}-\varepsilon^{2} \delta_{P} H_{P}-\varepsilon^{3} \delta_{H} H_{O} H_{P}, \\
\varepsilon^{3} \frac{d H_{O}}{d t} & =\delta_{O}+k_{-2} C-k_{2} H_{O} I_{L}-\varepsilon^{3} \delta_{O} H_{O}, \\
\varepsilon^{2} \frac{d R}{d t} & =\delta_{H} H_{O} H_{P}-\delta_{R} R \\
\frac{d C}{d t} & =k_{2} H_{O} I_{L}-\left(k_{-2}+\delta_{C}\right) C \\
\frac{d I_{T}}{d t} & =-\alpha_{3}\left(I_{T}-\mu_{3} I_{B}\right)-\delta_{I_{T}} I_{T}, \\
\varepsilon \frac{d I_{B}}{d t} & =\alpha_{2}\left(I_{L}-\mu_{2} I_{B}\right)+\alpha_{3}\left(I_{T}-\mu_{2} I_{B}\right)-\varepsilon I_{B}, \\
\varepsilon \frac{d I_{L}}{d t} & =-\alpha_{2} v\left(I_{L}-\mu_{2} I_{B}\right)-\varepsilon\left(k_{2} H_{O} I_{L}-k_{-2} C+\delta_{I_{L}} I_{L}\right) .
\end{aligned}
$$

At leading order the equations representing the inhibitor solve to give

$$
I_{T_{0}}=\Theta e^{-\delta_{I_{t}} t}, \quad I_{B_{0}}=\frac{\Theta}{\mu_{3}} e^{-\delta_{I_{t}} t}, \quad I_{L_{0}}=\frac{\Theta \mu_{2}}{\mu_{3}} e^{-\delta_{I_{t}} t} .
$$

Adding the leading order equations for $C$ and $H_{O}$ leads to

$$
\frac{d C_{0}}{d t}=\delta_{O}-\delta_{C} C_{0} \Rightarrow C_{0}=\frac{\delta_{O}}{\delta_{C}}+\left(1-\frac{\delta_{O}}{\delta_{C}}\right) e^{-\delta_{C} t}
$$

where matching implies $C_{0}(0)=1$, which gives

$$
H_{O_{0}}=\frac{\mu_{3}}{k_{2} \mu_{2} \Theta}\left(\delta_{O}\left(1+\frac{k_{-2}}{\delta_{C}}\right)+\left(1-\frac{\delta_{O}}{\delta_{C}}\right) e^{-\delta_{C} t}\right) e^{\delta_{I T} t}
$$

Adding $\varepsilon v \times$ the $T_{T}$ and $T_{B}$ equations with those of $T_{L}$ and $H_{P}$ leads to, at leading order,

$$
\frac{d T_{T_{0}}}{d t}=\frac{S_{1}}{v}-\delta_{T_{T}} T_{T_{0}}
$$

hence

$$
\begin{gathered}
T_{T_{0}}=\frac{S_{1}}{v \delta_{T_{T}}}\left(1-e^{-\delta_{T_{T}} t}\right), \quad T_{B_{0}}=\frac{S_{1}}{v \alpha_{4} \mu_{4}}+\frac{S_{1}}{v \delta_{T_{T}} \mu_{4}}\left(1-e^{-\delta_{T_{T}} t}\right), \\
T_{L_{0}}=\frac{S_{1}\left(\alpha_{1} \mu_{1}+\alpha_{4} \mu_{4}\right)}{v \alpha_{1} \alpha_{4} \mu_{4}}+\frac{S_{1} \mu_{1}}{v \delta_{T_{T}} \mu_{4}}\left(1-e^{-\delta_{T_{T}} t}\right) \\
H_{P_{0}}=\frac{S_{1} k_{-1}\left(\alpha_{1} \mu_{1}+\alpha_{4} \mu_{4}\right)}{k_{1} v \alpha_{1} \alpha_{4} \mu_{4}}+\frac{S_{1} k_{-1} \mu_{1}}{k_{1} v \delta_{T_{T}} \mu_{4}}\left(1-e^{-\delta_{T} t}\right) .
\end{gathered}
$$

Finally, at leading order, $R_{0}=\delta_{H} H_{O} H_{P} / \delta_{R}$. 
As stated in the main text, the maximum value for $T_{B}$ in the numerical solution is rather poorly predicted by the leading order approximation of the maximum corresponding to $T_{B_{0}}$ as $t \rightarrow \infty$, namely

$$
T_{B_{0}} \sim \frac{S_{1}}{v \mu_{4}}\left(\frac{1}{\alpha_{4}}+\frac{1}{\delta_{T_{T}}}\right)=T_{B_{\infty}}^{[11]}
$$

where for $\varepsilon \approx 0.04$ the error is about $40-50 \%$. This cannot be explained from the leading order expansion, and examination of the correction terms is needed to see why such an error can arise. Continuing on to the first correction term for $T_{B}$, we find that,

$$
\frac{d T_{B_{1}}}{d t}=-\delta_{T_{T}} T_{B_{1}}-\delta_{T_{T}} T_{B_{1 \infty}}^{[11]}+A_{0}^{[11]} e^{-\delta_{T_{T}} t}
$$

where

$$
\begin{aligned}
& T_{B_{1 \infty}}^{[11]}=-\frac{S_{1}\left(\alpha_{4}+\delta_{T_{T}}\right)\left(\left(\delta_{T_{L}} k_{1}+\delta_{P} k_{-1}\right)\left(\alpha_{1} \mu_{1} \alpha_{4}+\delta_{T_{T}}\left(\alpha_{1} \mu_{1}+\alpha_{4} \mu_{4}\right)\right)+k_{1} \delta_{T_{B}} v \alpha_{1}\left(\alpha_{4}+\delta_{T_{T}}\right)\right)}{k_{1} v^{2} \delta_{T_{T}}^{2} \alpha_{1} \alpha_{4}^{2} \mu_{4}^{2}}+\frac{1}{\mu_{4}}, \\
& A_{0}^{[11]}=\frac{S_{1}\left(v k_{1}\left(\delta_{T_{B}}-\delta_{T_{T}}\right)+\mu_{1}\left(k_{1}\left(\delta_{T_{L}}-\delta_{T_{T}}\right)+k_{-1}\left(\delta_{P}-\delta_{T_{T}}\right)\right)\right)}{k_{1} v^{2} \delta_{T_{T}} \mu_{4}^{2}},
\end{aligned}
$$

for which we obtain the solution

$$
T_{B_{1}}=T_{B_{1_{\infty}}}^{[11]}+\left(A_{0}^{[11]} t+A_{1}^{[11]}\right) e^{-\delta_{T_{T}} t}
$$

where $A_{1}^{[11]}$ is a constant of integration that could be determined by matching with the correction terms of the previous timescale (not undertaken). Here, $T_{B_{1}} \rightarrow T_{B_{1 \infty}}^{[11]}$ as $t \rightarrow \infty$, which turns out to make up a large part of the error in the prediction of the maximum of $T_{B}$.

The other variables tends to the following at leading order,

$$
\begin{gathered}
T_{T_{0}} \sim \frac{S_{1}}{v \delta_{T_{T}}}=T_{T_{\infty}}^{[11]}, \quad T_{L_{0}} \sim \frac{S_{1}}{v \mu_{4}}\left(\frac{\mu_{1}}{\delta_{T_{T}}}+\frac{\mu_{1}}{\alpha_{4}}+\frac{\mu_{4}}{\alpha_{1}}\right)=T_{L_{\infty}}^{[11]}, \\
H_{P_{0}} \sim \frac{S_{1} k_{-1}}{k_{1} v \mu_{4}}\left(\frac{\mu_{1}}{\delta_{T_{T}}}+\frac{\mu_{1}}{\alpha_{4}}+\frac{\mu_{4}}{\alpha_{1}}\right)=H_{P_{\infty}}^{[11]}, \quad C_{0} \sim \frac{\delta_{O}}{\delta_{C}} .
\end{gathered}
$$

as $t \rightarrow \infty$. The blood tyrosine solution will be part of that used to fit with data. The decay rate of inhibitor will also be matched with data.

The inhibitor, HPPD and homogentisic acid either decays or grows exponentially and there is shift in balance when $t=\ln (1 / \varepsilon) / \delta_{I_{T}}+O(1)$.

Timescale 12: $t=\eta_{12}(\varepsilon)+O(1)$

Corresponds to about $t=O(2)$ days. Here, $\eta_{12}(\varepsilon)=\left(1 / \delta_{I_{T}}\right) \ln (1 / \varepsilon)$ we rescale time such that $t \mapsto \eta_{12}(\varepsilon)+$ $t$ and introduce

$$
\begin{aligned}
& T_{T} \mapsto \frac{T_{T}}{\varepsilon}, \quad T_{B} \mapsto \frac{T_{B}}{\varepsilon}, \quad T_{L} \mapsto \frac{T_{L}}{\varepsilon}, \quad H_{P} \mapsto \frac{H_{P}}{\varepsilon}, \quad H_{O} \mapsto \varepsilon^{2} H_{O}, \\
& R \mapsto \varepsilon R, \quad C \mapsto C, \quad I_{T} \mapsto \varepsilon I_{T}, \quad I_{B} \mapsto \varepsilon I_{B}, \quad I_{L} \mapsto \varepsilon I_{L},
\end{aligned}
$$


which when applied to system (2.8) result in the following

$$
\begin{aligned}
\frac{d T_{T}}{d t} & =-\delta_{T_{T}} T_{T}-\alpha_{4}\left(T_{T}-\mu_{4} T_{B}\right)+\varepsilon \delta_{T_{T}}, \\
\varepsilon \frac{d T_{B}}{d t} & =\alpha_{1}\left(T_{L}-\mu_{1} T_{B}\right)+\alpha_{4}\left(T_{T}-\mu_{4} T_{B}\right)-\varepsilon \delta_{T_{B}} T_{B}, \\
\varepsilon^{2} \frac{d T_{L}}{d t} & =k_{1} H_{P}-k_{-1} T_{L}+\varepsilon\left(S_{1}-\alpha_{1} v\left(T_{L}-\mu_{1} T_{B}\right)\right)-\varepsilon^{2} \delta_{T_{L}} T_{L}, \\
\varepsilon^{2} \frac{d H_{P}}{d t} & =-k_{1} H_{P}+k_{-1} T_{L}-\varepsilon^{2}\left(\delta_{P} H_{P}-\delta_{H} H_{O} H_{P}\right), \\
\varepsilon^{2} \frac{d H_{O}}{d t} & =\delta_{O}+k_{-2} C-k_{2} H_{O} I_{L}-\varepsilon^{2} \delta_{O} H_{O}, \\
\varepsilon^{2} \frac{d R}{d t} & =\delta_{H} H_{O} H_{P}-\delta_{R} R, \\
\frac{d C}{d t} & =k_{2} H_{O} I_{L}-\left(k_{-2}+\delta_{C}\right) C, \\
\frac{d I_{T}}{d t} & =-\alpha_{3}\left(I_{T}-\mu_{3} I_{B}\right)-\delta_{I_{T}} I_{T}, \\
\varepsilon \frac{d I_{B}}{d t} & =\alpha_{2}\left(I_{L}-\mu_{2} I_{B}\right)+\alpha_{3}\left(I_{T}-\mu_{2} I_{B}\right)-\varepsilon I_{B}, \\
\varepsilon \frac{d I_{L}}{d t} & =-\alpha_{2} v\left(I_{L}-\mu_{2} I_{B}\right)-k_{2} H_{O} I_{L}+k_{-2} C-\varepsilon \delta_{I_{L}} I_{L} .
\end{aligned}
$$

By matching, at leading order the following are constant

$$
T_{T_{0}}=T_{T_{\infty}}^{[11]}, \quad T_{B_{0}}=T_{B_{\infty}}^{[11]}, \quad T_{L_{0}}=T_{L_{\infty}}^{[11]}, \quad H_{P_{0}}=H_{P_{\infty}}^{[11]}, \quad C_{0}=\frac{\delta_{O}}{\delta_{C}} .
$$

Adding the ODEs for $I_{T}, I_{B}, I_{L} / v$ and $C / v$ leads to

$$
\frac{d I_{T_{0}}}{d t}=\frac{\delta_{O}}{v}-\delta_{I_{T}} I_{T_{0}}
$$

which provides on solution

$$
\begin{aligned}
I_{L_{0}} & =\frac{\Theta \mu_{2}}{\mu_{3}} e^{-\delta_{I_{T}} t}-\frac{\delta_{O}}{v \mu_{3}}\left(\frac{\mu_{2}}{\delta_{I_{T}}}+\frac{\mu_{2}}{\alpha_{3}}+\frac{\mu_{3}}{\alpha_{2}}\right), \\
I_{B_{0}} & =\frac{\Theta}{\mu_{3}} e^{-\delta_{I_{T}} t}-\frac{\delta_{O}}{v \mu_{3}}\left(\frac{1}{\delta_{T_{T}}}+\frac{1}{\alpha_{3}}\right)=\frac{I_{L_{0}}}{\mu_{2}}+\frac{\delta_{O}}{v \alpha_{2} \mu_{2}}=\frac{I_{L_{0}}}{\mu_{2}}+I_{B_{c}}^{[12]}, \\
I_{T_{0}} & =\Theta e^{-\delta_{I_{T}} t}-\frac{\delta_{O}}{v \delta_{I_{T}}}=\frac{\mu_{3}}{\mu_{2}} I_{L_{0}}+\frac{\delta_{O}\left(\alpha_{2} \mu_{2}+\alpha_{3} \mu_{3}\right)}{v \alpha_{2} \mu_{2} \alpha_{3}}=\frac{\mu_{3}}{\mu_{2}} I_{L_{0}}+I_{T_{C}}^{[12]}, \\
H_{O_{0}} & =\frac{\delta_{O}\left(\delta_{C}+k_{-2}\right)}{k_{2} \delta_{C}\left(\frac{\Theta \mu_{2}}{\mu_{3}} e^{-\delta_{I_{T}} t}-\frac{\delta_{O}}{v \mu_{3}}\left(\frac{\mu_{2}}{\delta_{I_{T}}}+\frac{\mu_{2}}{\alpha_{3}}+\frac{\mu_{3}}{\alpha_{2}}\right)\right)}, \\
R_{0} & \left.=\frac{S_{1} k_{-1} \delta_{H} \delta_{O}\left(\delta_{C}+k_{-2}\right)}{\delta_{R} \delta_{C} k_{1} v \mu_{4} k_{2}\left(\frac{\Theta \mu_{2}}{\mu_{3}} e^{-\delta_{I_{T}} t}-\frac{\delta_{O}}{v \mu_{3}}\left(\frac{\mu_{2}}{\delta_{I_{T}}}+\frac{\mu_{2}}{\alpha_{3}}+\frac{\mu_{3}}{\alpha_{2}}\right)\right)}+\frac{\mu_{1}}{\alpha_{4}}+\frac{\mu_{4}}{\alpha_{1}}\right) .
\end{aligned}
$$


The inhibitor is continuing to drop and HPPD levels are rising, but still low so that tyrosine levels remains at their peak. Breakdown of the current balance occurs when, as it turns out,

$$
\frac{\Theta \mu_{2}}{\mu_{3}} e^{-\delta_{I_{T}} t}-\frac{\delta_{O}}{v \mu_{3}}\left(\frac{\mu_{2}}{\delta_{I_{T}}}+\frac{\mu_{2}}{\alpha_{3}}+\frac{\mu_{3}}{\alpha_{2}}\right)=O\left(\varepsilon^{2 / 3}\right),
$$

i.e.

$$
t=\frac{1}{\delta_{I_{T}}} \ln \left(\frac{\Theta v \alpha_{2} \mu_{2} \alpha_{3} \delta_{I_{T}}}{\delta_{O}\left(\alpha_{2} \alpha_{3}+\delta_{I_{T}}\left(\alpha_{2} \mu_{2}+\alpha_{3} \mu_{3}\right)\right)}\right)+O\left(\varepsilon^{2 / 3}\right)
$$

Timescale 13: $t=\eta_{12}(\varepsilon)+\eta_{13}(\varepsilon)+O\left(\varepsilon^{2 / 3}\right)$

Corresponds to about $t \approx 3$ days. Here, $\eta_{13}(\varepsilon)$ is such that

$$
\eta_{13}(0)=\left(1 / \delta_{I_{T}}\right) \ln \left[\Theta v \alpha_{2} \mu_{2} \alpha_{3} \delta_{I_{T}} / \delta_{O}\left(\alpha_{2} \mu_{2} \alpha_{3}+\delta_{I_{T}}\left(\alpha_{2} \mu_{2}+\alpha_{3} \mu_{3}\right)\right)\right] .
$$

We rescale time such that $t \mapsto \eta_{12}(\varepsilon)+\eta_{13}(\varepsilon)+\varepsilon^{2 / 3} t$, and obtain the scalings

$$
\begin{gathered}
T_{T} \mapsto \frac{T_{T}}{\varepsilon}, \quad T_{B} \mapsto \frac{T_{B}}{\varepsilon}, \quad T_{L} \mapsto \frac{T_{L}}{\varepsilon}, \quad H_{P} \mapsto \frac{H_{P}}{\varepsilon}, \quad H_{O} \mapsto \varepsilon^{4 / 3} H_{O}, \quad R \mapsto \varepsilon^{1 / 3} R, \\
C \mapsto C, \quad I_{T} \mapsto \varepsilon \overline{I_{T}}=\varepsilon I_{T_{c}}^{[12]}+\varepsilon^{5 / 3} I_{T}, \quad I_{B} \mapsto \varepsilon \overline{I_{B}}=\varepsilon I_{B_{c}}^{[12]}+\varepsilon^{5 / 3} I_{B}, \quad I_{L} \mapsto \varepsilon^{5 / 3} I_{L} .
\end{gathered}
$$

applying these to equations (2.8) to get

$$
\begin{aligned}
\frac{d T_{T}}{d t} & =-\varepsilon^{2 / 3}\left(\delta_{T_{T}} T_{T}+\alpha_{4}\left(T_{T}-\mu_{4} T_{B}\right)\right)+\varepsilon^{5 / 3} \delta_{T_{T}} \\
\varepsilon^{1 / 3} \frac{d T_{B}}{d t} & =\alpha_{1}\left(T_{L}-\mu_{1} T_{B}\right)+\alpha_{4}\left(T_{T}-\mu_{4} T_{B}\right)-\varepsilon \delta_{T_{B}} T_{B} \\
\varepsilon^{4 / 3} \frac{d T_{L}}{d t} & =k_{1} H_{P}-k_{-1} T_{L}+\varepsilon\left(S_{1}-\alpha_{1} v\left(T_{L}-\mu_{1} T_{B}\right)\right)-\varepsilon^{2} \delta_{T_{L}} T_{L} \\
\varepsilon^{4 / 3} \frac{d H_{P}}{d t} & =-k_{1} H_{P}+k_{-1} T_{L}-\varepsilon^{2}\left(\delta_{P} H_{P}-\delta_{H} H_{O} H_{P}\right) \\
\varepsilon^{2 / 3} \frac{d H_{O}}{d t} & =\delta_{O}+k_{-2} C-k_{2} H_{O} I_{L}-\varepsilon^{2} \delta_{O} H_{O} \\
\varepsilon^{4 / 3} \frac{d R}{d t} & =\delta_{H} H_{O} H_{P}-\delta_{R} R \\
\frac{d C}{d t} & =\varepsilon^{2 / 3}\left(k_{2} H_{O} I_{L}-\left(k_{-2}+\delta_{C}\right) C\right) \\
\frac{d I_{T}}{d t} & =-\alpha_{3}\left(I_{T_{c}}^{[12]}-\mu_{3} I_{B_{c}}^{[12]}\right)-\delta_{I_{T}} I_{T_{C}}^{[12]}-\varepsilon^{2 / 3}\left(\alpha_{3}\left(I_{T}-\mu_{3} I_{B}\right)+\delta_{I_{T}} I_{T}\right) \\
\varepsilon \frac{d I_{B}}{d t} & =\alpha_{3}\left(I_{T_{c}}^{[12]}-\mu_{3} I_{B_{c}}^{[12]}\right)-\alpha_{2} \mu_{2} I_{B_{c}}^{[12]}+\varepsilon^{2 / 3}\left(\alpha_{3}\left(I_{T}-\mu_{3} I_{B}\right)+\alpha_{2}\left(I_{L}-\mu_{2} I_{B}\right)\right)-\varepsilon \overline{I_{B}} \\
\varepsilon \frac{d I_{L}}{d t} & =v \alpha_{2} \mu_{2} I_{B_{c}}^{[12]}-k_{2} H_{O} I_{L}+k_{-2} C-\varepsilon^{2 / 3} v \alpha_{2}\left(I_{L}-\mu_{2} I_{B}\right)-\varepsilon^{5 / 3} \delta_{I_{L}} I_{L}
\end{aligned}
$$


To leading order, the following are constant,

$$
T_{T_{0}}=T_{T_{\infty}}^{[11]}, \quad T_{B_{0}}=T_{B_{\infty}}^{[11]}, \quad T_{L_{0}}=T_{L_{\infty}}^{[11]}, \quad H_{P_{0}}=H_{P_{\infty}}^{[11]}, \quad C_{0}=\frac{\delta_{O}}{\delta_{C}} .
$$

Integrating the tissue inhibitor equation gives, on matching,

$$
I_{T_{0}}=-\frac{\delta_{O}}{v \alpha_{2} \mu_{2} \alpha_{3}} r_{I}^{[13]} t
$$

where $\Upsilon_{I}^{[13]}=\alpha_{2} \mu_{2} \alpha_{3}+\delta_{I_{T}}\left(\alpha_{2} \mu_{2}+\alpha_{3} \mu_{3}\right)$. Once again the liver inhibitor and HPPD are in quasisteady state so that

$$
I_{L_{0}}=\frac{\delta_{O}+k_{-2} \delta_{O} / \delta_{C}}{k_{2} H_{O_{0}}}, \quad I_{B_{0}}=\frac{\alpha_{3} I_{T_{0}}+\alpha_{2} I_{L_{0}}}{\alpha_{2} \mu_{2}+\alpha_{3} \mu_{3}}
$$

Adding the ODEs for $H_{O}$ and $-I_{L}$ leads to, at $O\left(\varepsilon^{4 / 3}\right)$,

$$
\frac{d H_{O_{0}}}{d t}=-v \alpha_{2}\left(\mu_{2} I_{B_{0}}-I_{L_{0}}\right)
$$

which eventually yields

$$
\frac{d H_{O_{0}}}{d t}=\frac{\delta_{O} \Upsilon_{I}^{[13]}}{\alpha_{2} \mu_{2}+\alpha_{3} \mu_{3}} t+\frac{v \alpha_{2} \alpha_{3} \mu_{3}\left(\delta_{O}+k_{-2} \delta_{O} / \delta_{C}\right)}{k_{2}\left(\alpha_{2} \mu_{2}+\alpha_{3} \mu_{3}\right)} \frac{1}{H_{O_{0}}} .
$$

This has solutions in terms of Airy functions and matching with Timescale 12 solutions means that $H_{O_{0}} \sim(-t)^{-1} v \alpha_{2} \alpha_{3} \mu_{3}\left(1+k_{-2} / \delta_{C}\right) / k_{2} \Upsilon_{I}^{[13]}$ as $t \rightarrow-\infty$. Given the solution $H_{O}$, then

$$
I_{L_{0}}=\frac{\delta_{O}+k_{-2} \delta_{O} / \delta_{C}}{k_{2} H_{O_{0}}}, \quad I_{B_{0}}=\frac{\alpha_{2}\left(\delta_{O}+k_{-2} \delta_{O} / \delta_{C}\right)}{k_{2}\left(\alpha_{2} \mu_{2}+\alpha_{3} \mu_{3}\right) H_{O_{0}}}-\frac{\delta_{O} \Upsilon_{I}^{[13]}}{v \alpha_{2} \mu_{2}\left(\alpha_{2} \mu_{2}+\alpha_{3} \mu_{3}\right)} t
$$

and $R_{0}=\delta_{H} H_{O_{0}} H_{P_{0}}^{[11]} / \delta_{R}$.

In large time we have

$$
H_{O_{0}} \sim \frac{\delta_{O} \Upsilon_{I}^{[13]}}{2\left(\alpha_{2} \mu_{2}+\alpha_{3} \mu_{3}\right)} t^{2}, \quad I_{L_{0}} \sim \frac{2 \alpha_{2}\left(\alpha_{2} \mu_{2}+\alpha_{3} \mu_{3}\right)\left(\delta_{O}+k_{-2} \delta_{O} / \delta_{C}\right)}{k_{2} \delta_{O} \Upsilon_{I}^{[13]}} \frac{1}{t^{2}}
$$

and $I_{T_{0}}=O(1 / t)$ and $I_{B_{0}}=O(1 / t)$.

Inhibitor concentrations fall rapidly in the liver as HPPD recovers over this fast timescale. Tyrosine and HPPA concentrations are static and homogenistic acid increases to near pretreated state. It turns out there is a shift in balance when $t=O\left(\varepsilon^{-1 / 6}\right)$ when $H_{O_{0}}=O\left(\varepsilon^{-1 / 3}\right)$ and $I_{L_{0}}=O\left(\varepsilon^{-1 / 3}\right)$.

Timescale 14: $t=\eta_{12}(\varepsilon)+\eta_{13}(\varepsilon)+O\left(\varepsilon^{1 / 2}\right)$

Corresponds to about $t \approx 3$ days. We introduce the scalings: $t \mapsto \eta_{12}(\varepsilon)+\eta_{13}(\varepsilon)+\varepsilon^{1 / 2} t$,

$$
\begin{gathered}
T_{T} \mapsto \frac{T_{T}}{\varepsilon}, \quad T_{B} \mapsto \frac{T_{B}}{\varepsilon}, \quad T_{L} \mapsto \frac{T_{L}}{\varepsilon}, \quad H_{P} \mapsto \frac{H_{P}}{\varepsilon}, \quad H_{O} \mapsto \varepsilon H_{O}, \quad R \mapsto R, \\
C \mapsto C, \quad I_{T} \mapsto \varepsilon \overline{I_{T}}=\varepsilon I_{T_{C}}^{[12]}+\varepsilon^{3 / 2} I_{T}, \quad I_{B} \mapsto \varepsilon \overline{I_{B}}=\varepsilon I_{B_{c}}^{[12]}+\varepsilon^{3 / 2} I_{B}, \quad I_{L} \mapsto \varepsilon^{2} I_{L}, \quad \text { A. } 114
\end{gathered}
$$


and apply them to equations (2.8) resulting in the system

$$
\begin{aligned}
\frac{d T_{T}}{d t} & =-\varepsilon^{1 / 2}\left(\delta_{T_{T}} T_{T}+\alpha_{4}\left(T_{T}-\mu_{4} T_{B}\right)\right)+\varepsilon^{3 / 2} \delta_{T_{T}} \\
\varepsilon^{1 / 2} \frac{d T_{B}}{d t} & =\alpha_{1}\left(T_{L}-\mu_{1} T_{B}\right)+\alpha_{4}\left(T_{T}-\mu_{4} T_{B}\right)-\varepsilon \delta_{T_{B}} T_{B} \\
\varepsilon^{3 / 2} \frac{d T_{L}}{d t} & =k_{1} H_{P}-k_{-1} T_{L}+\varepsilon\left(S_{1}-\alpha_{1} v\left(T_{L}-\mu_{1} T_{B}\right)\right)-\varepsilon^{2} \delta_{T_{L}} T_{L} \\
\varepsilon^{3 / 2} \frac{d H_{P}}{d t} & =-k_{1} H_{P}+k_{-1} T_{L}+\varepsilon \delta_{H} H_{O} H_{P}-\varepsilon^{2} \delta_{P} H_{P} \\
\varepsilon^{1 / 2} \frac{d H_{O}}{d t} & =\delta_{O}+k_{-2} C-k_{2} H_{O} I_{L}-\varepsilon \delta_{O} H_{O} \\
\varepsilon^{3 / 2} \frac{d R}{d t} & =\delta_{H} H_{O} H_{P}-\delta_{R} R \\
\frac{d C}{d t} & =\varepsilon^{1 / 2}\left(k_{2} H_{O} I_{L}-\left(\delta_{C}+k_{-2}\right) C\right) \\
\frac{d I_{T}}{d t} & =-\alpha_{3}\left(I_{T_{c}}^{[12]}-\mu_{3} I_{B_{c}}^{[12]}\right)-\delta_{I_{T}} I_{T_{c}}^{[12]}-\varepsilon^{1 / 2}\left(\alpha_{3}\left(I_{T}-\mu_{3} I_{B}\right)+\delta_{\left.I_{T} I_{T}\right)}\right. \\
\varepsilon \frac{d I_{B}}{d t} & =\alpha_{3}\left(I_{T_{c}}^{[12]}-\mu_{3} I_{B_{c}}^{[12]}\right)-\alpha_{2} \mu_{2} I_{B_{c}}^{[12]}+\varepsilon^{1 / 2}\left(\alpha_{3}\left(I_{T}-\mu_{3} I_{B}\right)+\alpha_{2}\left(I_{L}-\mu_{2} I_{B}\right)\right)-\varepsilon \overline{I_{B}} \\
\varepsilon^{3 / 2} \frac{d I_{L}}{d t} & =v \alpha_{2} \mu_{2} I_{B_{c}}^{[12]}-k_{2} H_{O} I_{L}+k_{-2} C+\varepsilon^{1 / 2} v \alpha_{2} \mu_{2} I_{B}-\varepsilon v \alpha_{2} I_{L}-\varepsilon^{2} \delta_{I_{L}} I_{L}
\end{aligned}
$$

To leading order, the following are constant

$$
T_{T_{0}}=T_{T_{\infty}}^{[11]}, \quad C_{0}=\frac{\delta_{O}}{\delta_{C}}
$$

By matching

$$
I_{T_{0}}=-\frac{\delta_{O} \Upsilon_{I}^{[13]}}{v \alpha_{2} \mu_{2} \alpha_{3}} t, \quad I_{B_{0}}=-\frac{\delta_{O} \Upsilon_{I}^{[13]}}{v \alpha_{2} \mu_{2}\left(\alpha_{2} \mu_{2}+\alpha_{3} \mu_{3}\right)} t
$$

and, in particular,

$$
H_{O_{0}}=\frac{\delta_{O} \Upsilon_{I}^{[13]}}{2\left(\alpha_{2} \mu_{2}+\alpha_{3} \mu_{3}\right)} t^{2}, \quad I_{L_{0}}=\frac{2\left(\alpha_{2} \mu_{2}+\alpha_{3} \mu_{3}\right) k_{-2}}{k_{2} \delta_{C} \Upsilon_{I}^{[13]}} \frac{1}{t^{2}}
$$

i.e. the large time solutions of the previous timescale continue on. With tissue tyrosine being constant, the blood/liver tyrosine and HPPA satisfy the simultaneous equation

$$
\begin{gathered}
\left(\alpha_{1} \mu_{1}+\alpha_{4} \mu_{4}\right) T_{B_{0}}-\alpha_{1} T_{L_{0}}=\frac{S_{1} \alpha_{4}}{v \delta_{T_{T}}}, \quad k_{1} H_{P_{0}}-k_{-1} T_{L_{0}}=0 \\
v \alpha_{1} \mu_{1} T_{B_{0}}-v \alpha_{1} T_{L_{0}}-\delta_{H} H_{O_{0}} H_{P_{0}}=-S_{1}
\end{gathered}
$$


where $H_{O_{0}}$ is given above, which, expressing the solutions in terms of the matched initial conditions, solves to give

$$
T_{B_{0}}=\frac{1+\psi \Psi_{0}^{[14]} t^{2}}{1+\Psi_{0}^{[14]}, t^{2}} T_{B_{\infty}}^{[11]}, \quad T_{L_{0}}=\frac{1}{1+\Psi_{0}^{[14]} t^{2}} T_{L_{\infty}}^{[11]}, \quad H_{P_{0}}=\frac{1}{1+\Psi_{0}^{[14]} t^{2}} H_{P_{\infty}}^{[11]}
$$

where

$$
\psi^{[14]}=\frac{\alpha_{4}^{2} \mu_{4}}{\left(\alpha_{4}+\delta_{T_{T}}\right)\left(\alpha_{1} \mu_{1}+\alpha_{4} \mu_{4}\right)}, \quad \Psi_{0}^{[14]}=\frac{\delta_{O} \delta_{H} k_{-1} \Upsilon_{I}^{[13]}\left(\alpha_{1} \mu_{1}+\alpha_{4} \mu_{4}\right)}{2 k_{1} v \alpha_{1} \alpha_{4} \mu_{4}\left(\alpha_{2} \mu_{2}+\alpha_{3} \mu_{3}\right)},
$$

noting that $\psi^{[14]}<1$. Finally for homogentisic acid

$$
R_{0}=\frac{\delta_{O} \delta_{H} \Upsilon_{I}^{[13]} H_{P_{\infty}}^{[11]}}{2 \delta_{R}\left(\alpha_{2} \mu_{2}+\alpha_{3} \mu_{3}\right)} \frac{t^{2}}{\left(1+\Psi_{0} t^{2}\right)}
$$

In large time we have

$$
\begin{gathered}
T_{B_{0}} \sim \psi^{[14]} T_{B_{\infty}}^{[11]}=T_{B_{\infty}}^{[14]}, \quad T_{L_{0}} \sim \frac{T_{L_{\infty}}^{[11]}}{\Psi_{0}^{[14]}} \frac{1}{t^{2}}, \quad H_{P_{0}} \sim \frac{H_{P_{\infty}}^{[11]}}{\Psi_{0}^{[14]}} \frac{1}{t^{2}}, \\
R_{0} \sim \frac{\delta_{O} \delta_{H} \Upsilon_{I}^{[13]} H_{P_{\infty}}^{[11]}}{2 \delta_{R} \Psi_{0}\left(\alpha_{2} \mu_{2}+\alpha_{3} \mu_{3}\right)}=R_{\infty}^{[14]} .
\end{gathered}
$$

As HPPD is increasing the liver tyrosine and HPPA start to decline. The approximations breakdown in this timescale at $t=O\left(\varepsilon^{-1 / 2}\right)$, when $T_{L}$ and $H_{P}$ are reduced by $O(\varepsilon)$ and $H_{O}$ increase by $O(1 / \varepsilon)$.

Timescale 15: $t=\eta_{12}(\varepsilon)+\eta_{13}(\varepsilon)+O(1)$

Corresponds to about $t \approx 3-4$ days. We rescale time such that $t \mapsto \eta_{12}(\varepsilon)+\eta_{13}(\varepsilon)+t$ and introduce the scalings

$$
\begin{aligned}
T_{T} & \mapsto \frac{T_{T}}{\varepsilon}, \quad T_{B} \mapsto \frac{T_{B}}{\varepsilon}, \quad T_{L} \mapsto T_{L}, \quad H_{P} \mapsto H_{P}, \quad H_{O} \mapsto H_{O}, \\
R & \mapsto R, \quad C \mapsto C, \quad I_{T} \mapsto \varepsilon I_{T}, \quad I_{B} \mapsto \varepsilon I_{B}, \quad I_{L} \mapsto \varepsilon^{3} I_{L} .
\end{aligned}
$$

Applying these to the equations (2.8) we obtain

$$
\begin{aligned}
\frac{d T_{T}}{d t} & =-\delta_{T_{T}} T_{T}-\alpha_{4}\left(T_{T}-\mu_{4} T_{B}\right)+\varepsilon \delta_{T_{T}}, \\
\varepsilon \frac{d T_{B}}{d t} & =-\alpha_{1} \mu_{1} T_{B}+\alpha_{4}\left(T_{T}-\mu_{4} T_{B}\right)+\varepsilon\left(\alpha_{1} T_{L}-\delta_{T_{B}} T_{B}\right), \\
\varepsilon^{2} \frac{d T_{L}}{d t} & =S_{1}+k_{1} H_{P}-k_{-1} T_{L}-\alpha_{1} v\left(T_{L}-\mu_{1} T_{B}\right)-\varepsilon^{2} \delta_{T_{L}} T_{L}, \\
\varepsilon^{2} \frac{d H_{P}}{d t} & =-k_{1} H_{P}+k_{-1} T_{L}+\delta_{H} H_{O} H_{P}-\varepsilon^{2} \delta_{P} H_{P},
\end{aligned}
$$




$$
\begin{aligned}
\frac{d H_{O}}{d t} & =\delta_{O}+k_{-2} C-k_{2} H_{O} I_{L}-\delta_{O} H_{O}, \\
\varepsilon^{2} \frac{d R}{d t} & =\delta_{H} H_{O} H_{P}-\delta_{R} R, \\
\frac{d C}{d t} & =k_{2} H_{O} I_{L}-\left(k_{-2}+\delta_{C}\right) C, \\
\frac{d I_{T}}{d t} & =-\alpha_{3}\left(I_{T}-\mu_{3} I_{B}\right)-\delta_{I_{T}} I_{T}, \\
\varepsilon \frac{d I_{B}}{d t} & =\alpha_{3}\left(I_{T}-\mu_{3} I_{B}\right)-\mu_{2} I_{B}-\varepsilon I_{B}+\varepsilon^{2} \alpha_{2} I_{L}, \\
\varepsilon^{3} \frac{d I_{L}}{d t} & =v \alpha_{2} \mu_{2} I_{B}-k_{2} H_{O} I_{L}+k_{-2} C-\varepsilon^{2} v \alpha_{2} I_{L}-\varepsilon^{3} \delta_{I_{L}} I_{L} .
\end{aligned}
$$

Combining the first two equations leads to

$$
T_{T_{0}}=T_{T_{\infty}}^{[11]} e^{-\Omega_{T}^{[15]} t}, \quad T_{B_{0}}=T_{B_{\infty}}^{[14]} e^{-\Omega_{T}^{[15]} t},
$$

where $\Omega_{T}^{[15]}=\left(\alpha_{1} \mu_{1} \alpha_{4}+\delta_{T_{T}}\left(\alpha_{1} \mu_{1}+\alpha_{4} \mu_{4}\right)\right) /\left(\alpha_{1} \mu_{1}+\alpha_{4} \mu_{4}\right)$ and recalling that $T_{T_{\infty}}^{[11]}=S_{1} / v \delta_{T_{T}}$ and

$$
T_{B_{\infty}}^{[14]}=\psi^{[14]} \frac{S_{1}\left(\alpha_{4}+\delta_{T_{T}}\right)}{\delta_{T_{T}} v \alpha_{4} \mu_{4}}=\frac{S_{1} \alpha_{4}}{\delta_{T_{T}} v\left(\alpha_{1} \mu_{1}+\alpha_{4} \mu_{4}\right)} .
$$

Combining equations for $I_{T}$ and $I_{B}$ gives

$$
I_{T_{0}}=I_{T_{c}}^{[12]} e^{-\Omega_{I}^{[15]} t}, \quad I_{B_{0}}=I_{B_{c}}^{[12]} e^{-\Omega_{I}^{[15]} t},
$$

where $\Omega_{I}^{[15]}=\left(\alpha_{2} \mu_{2} \alpha_{3}+\delta_{I_{T}}\left(\alpha_{2} \mu_{2}+\alpha_{3} \mu_{3}\right)\right) /\left(\alpha_{2} \mu_{2}+\alpha_{3} \mu_{3}\right), I_{B_{c}}^{[12]}=\delta_{O} / v \alpha_{2} \mu_{2}$ and $I_{T_{c}}^{[12]}=\delta_{O}\left(\alpha_{2} \mu_{2}+\right.$ $\left.\alpha_{3} \mu_{3}\right) / v \alpha_{2} \mu_{2} \alpha_{3}$. Adding the ODEs for $H_{O}$ and $-I_{L}$ yields

$$
\frac{d H_{O_{0}}}{d t}=\delta_{O}\left(1-H_{O_{0}}\right)+v \alpha_{2} \mu_{2} I_{B_{0}} \quad \Rightarrow \quad H_{O_{0}}=1-\frac{\phi(t)}{\phi(0)}
$$

using $I_{B_{c}}^{[12]}=\delta_{O} / v \alpha_{2} \mu_{2}$ and where

$$
\phi(t)=\Omega_{I}^{[15]} e^{-\delta_{O} t}-\delta_{O} e^{-\Omega_{I}^{[15]} t},
$$

noting that $\phi(t) \rightarrow 0$ as $t \rightarrow \infty$. Likewise adding $C$ and $I_{L}$ gives

$$
\frac{d C_{0}}{d t}=v \alpha_{2} \mu_{2} I_{B_{0}}-\delta_{C} C_{0} \Rightarrow C_{0}=\frac{\delta_{O}}{\delta_{C}} \frac{\phi_{c}(t)}{\phi_{c}(0)}
$$

using the matching condition $C_{0}(0)=\delta_{O} / \delta_{C}$ and where $\phi_{c}(t)=\Omega_{I}^{[15]} e^{-\delta_{C} t}-\delta_{C} e^{-\Omega_{I}^{[15]} t}$, noting that $\phi_{c}(t) \rightarrow 0$ as $t \rightarrow \infty$. Between the equations for $H_{P}$ and $T_{L}$ we have

$$
\begin{aligned}
& H_{P_{0}}=H_{P_{0}}^{S S}+\frac{S_{1} \phi(t)+v \alpha_{1} \mu_{1} \phi(0) T_{B_{0}}^{[14]} e^{-\Omega_{T}^{[15]} t}}{\delta_{H}(\phi(0)-\phi(t))}, \\
& T_{L_{0}}=T_{L_{0}}^{S S}+\frac{S_{1} k_{1} \phi(t)+v \alpha_{1} \mu_{1} T_{B_{0}}^{[14]} e^{-\Omega_{T}^{[15]} t}\left(\phi(0)\left(\delta_{H}+k_{1}\right)-\delta_{H} \phi(t)\right)}{\delta_{H} k_{-1}(\phi(0)-\phi(t))} .
\end{aligned}
$$


For homogentisic acid,

$$
R_{0}=R_{0}^{S S}-\frac{\delta_{H}}{\delta_{R}}\left(\frac{\phi(t)}{\phi(0)} H_{P_{0}}^{S S}+\left(1-\frac{\phi(t)}{\phi(0)}\right)\left(\frac{S_{1} \phi(t)+v \alpha_{1} \mu_{1} \phi(0) T_{B_{0}}^{[14]} e^{-\Omega_{T}^{[15]} t}}{\delta_{H}(\phi(0)-\phi(t))}\right)\right) .
$$

To leading order $I_{L}$ satisfies

$$
I_{L_{0}}=\frac{v \alpha_{2} \mu_{2} I_{B_{c}}^{[12]} e^{-\Omega_{I}^{[15]} t}+k_{-2} \delta_{O} \phi_{c}(t) / \delta_{C} \phi_{c}(0)}{k_{2}(1-\phi(t) / \phi(0))}
$$

In large time we have

$$
T_{L_{0}} \sim T_{L_{0}}^{S S}, \quad H_{P_{0}} \sim H_{P_{0}}^{S S}, \quad H_{O_{0}} \sim 1, \quad R_{0} \sim R_{0}^{S S},
$$

i.e. at their pretreated concentrations. Inhibitor and complex concentrations are such that they have negligible effects at leading order. Blood and tissue tyrosine concentrations are in decline, but still high. The blood and tissue tyrosine decay exponentially and eventually reaches the order of magnitude of the pretreated state in $t=\ln (1 / \varepsilon) / \Omega_{T}^{[15]}+O(1)$.

Timescale 16: $t=\eta_{12}(\varepsilon)+\eta_{13}(\varepsilon)+\eta_{16}(\varepsilon)+O(1)$

Corresponds to about $t=O(4)$ days. Here, $\eta_{16}(\varepsilon)=\ln (1 / \varepsilon) / \Omega_{T}^{[15]}=\left(\alpha_{1} \mu_{1}+\alpha_{4} \mu_{4}\right) \ln (1 / \varepsilon) /\left(\alpha_{1} \mu_{1} \alpha_{4}+\right.$ $\left.\delta_{T_{T}}\left(\alpha_{1} \mu_{1}+\alpha_{4} \mu_{4}\right)\right)$, we rescale time such that $t \mapsto \eta_{12}(\varepsilon)+\eta_{13}(\varepsilon)+\eta_{16}(\varepsilon)+t$ and introduce the scalings

$$
\begin{gathered}
T_{T} \mapsto T_{T}, T_{B} \mapsto T_{B}, T_{L} \mapsto T_{L}, H_{P} \mapsto H_{P}, H_{O} \mapsto H_{O}, R \mapsto R, C \mapsto \varepsilon^{\min \left\{\Omega_{I}^{[15]}, \delta_{C}\right\} / \Omega_{T}^{[15]}} C, \\
I_{T} \mapsto \varepsilon^{1+\Omega_{I}^{[15]} / \Omega_{T}^{[15]}} I_{T}, \quad I_{B} \mapsto \varepsilon^{1+\Omega_{I}^{[15]} / \Omega_{T}^{[15]}} I_{B}, \quad I_{L} \mapsto \varepsilon^{3+\min \left\{\Omega_{I}^{[15]}, \delta_{C}\right\} / \Omega_{T}^{[15]}} I_{L} ; \quad \text { (A.139) }
\end{gathered}
$$

the scalings on the complex and inhibitor variables mean they make negligible contribution at leading order.

The following are at their pretreated concentrations

$$
T_{L_{0}}=T_{L_{0}}^{S S}, \quad H_{P_{0}}=H_{P_{0}}^{S S}, \quad H_{O_{0}}=1, \quad R_{0}=R_{0}^{S S},
$$

and the equations for the leading order blood and tissue tyrosine are

$$
\begin{aligned}
\frac{d T_{T}}{d t} & =\delta_{T_{T}}\left(1-T_{T}\right)-\alpha_{4}\left(T_{T}-\mu_{4} T_{B}\right), \\
\varepsilon \frac{d T_{B}}{d t} & =\alpha_{1}\left(T_{L}-\mu_{1} T_{B}\right)+\alpha_{4}\left(T_{T}-\mu_{4} T_{B}\right)-\varepsilon \delta_{T_{B}} T_{B},
\end{aligned}
$$

whereby solving and matching gives

$$
\begin{aligned}
& T_{T_{0}}=T_{T_{0}}^{S S}+\frac{S_{1}}{v \delta_{T_{T}}} e^{-\Omega_{T}^{[15]} t} \\
& T_{B_{0}}=T_{B_{0}}^{S S}+\frac{S_{1} \alpha_{4}}{\delta_{T_{T}} v\left(\alpha_{1} \mu_{1}+\alpha_{4} \mu_{4}\right)} e^{-\Omega_{T}^{[15]} t}
\end{aligned}
$$

recalling that $\Omega_{T}^{[15]}=\left(\alpha_{1} \mu_{1} \alpha_{4}+\delta_{T_{T}}\left(\alpha_{1} \mu_{1}+\alpha_{4} \mu_{4}\right)\right) /\left(\alpha_{1} \mu_{1}+\alpha_{4} \mu_{4}\right)$ and hence $T_{T_{0}} \rightarrow T_{T_{0}}^{S S}$ and $T_{B_{0}} \rightarrow$ $T_{B_{0}}^{S S}$ as $t \rightarrow \infty$. 


\section{B. Animal Experimentation}

Male Han Wistar Crl:WI rats (Charles River UK Ltd., Kent) were acclimatised for a period of 7 days prior to dosing and the non-GLP experimental design was based on OECD guidelines No. 417 for Toxicokinetics. Throughout the studies, the rats had access to domestic water and diet - PMI Nutrition International Certified Rodent Diet No. 5CR4 (14\% protein) — ad libitum. Husbandry practices and environmental enrichment were as Test Facility SOPs (Charles River UK Ltd., Edinburgh). All animals were checked each day for viability, received detailed clinical examination prior to dosing and were monitored for reaction to treatment.

Three rats, approximately 9 to 10 weeks old and weighing between 297 and $337 \mathrm{~g}$, were dosed with either vehicle only or $0.5 \mathrm{mg} / \mathrm{kg}, 1 \mathrm{mg} / \mathrm{kg}$ or $2 \mathrm{mg} / \mathrm{kg}$ nitisinone (S02F832242K Syngenta Ltd., Switzerland). The vehicle consisted of $0.5 \%$ concentration (w/v) carboxymethylcellulose $(\mathrm{CMC})$, medium viscosity (Sigma, UK) with $0.5 \%$ (v/v) Tween 80 (Sigma, Switzerland) in water, and the nitisinone (S02F832242K, Syngenta Ltd., Switzerland) formulations were prepared from a stock solution at 0.2 $\mathrm{mg} / \mathrm{mL}$, both prepared on the day of administration. Each dosing formulation was administered to the rats by a single oral gavage dose using a graduated syringe with a plastic cannula attached in a volume of $10 \mathrm{~mL} / \mathrm{kg}$.

Blood samples (approximately $100 \mu \mathrm{L}$ ) were collected from the lateral tail vein from each rat using a hypodermic needle into dry-coated potassium-EDTA vessels at the following time points after dosing: $0,0.5,1,2,4,8,12,24,36,48,72,96,120,144$ and $168 \mathrm{~h}$. Immediately following collection, $50 \mu \mathrm{L}$ of whole blood from each sample, was accurately measured into a plain plastic tube holding $50 \mu \mathrm{L}$ of Milli Q water. Tubes were placed on a roller mixer for approximately 1 minute and then frozen on dry ice pending storage at -80 degrees Celsius.

Samples were analysed under the test conditions set out by the laboratory (Bioanalytical Laboratory at Charles River, Edinburgh) for concentration of nitisinone and tyrosine after protein precipitation in either acetonitrile or acetone, respectively. Samples were analysed on a API4000, AB Sciex mass spectrometer with a micro HPLC pump and vacuum degasser (Series 200, Perkin Elmer), an autosampler (HTS Pal, CTC Analytics) and Analyst Version 1.4.2, AB Sciex data handling system. The samples were separated on a Kinetex PFP, 50 x $2.1 \mathrm{~mm}, 2.6 \mu \mathrm{m}$ Phenomenex column with a Prefit $0.5 \mu \mathrm{m}$ Anachan guard column using the following mobile phases: for nitisinone mobile phase A- acetonitrile / formic acid (100/0.1, v/v) and mobile phase B- $10 \mathrm{mM}$ ammonium acetate / formic acid (100/0.1, $\mathrm{v} / \mathrm{v}$ ), and for tyrosine mobile phase A- acetonitrile and mobile phase B- $10 \mathrm{mM}$ ammonium acetate / formic acid (100/0.1, v/v). Nifedipine was used as the internal standard for the nitisinone samples, whilst the tyrosine measurements were conducted in the absence of an internal standard. Sample concentrations of nitisinone and tyrosine were calculated from respective calibration curves in accordance to the acceptance criteria outline by the Bioanalytical Laboratory. 